\title{
The OPERA experiment in the CERN to Gran Sasso
} neutrino beam

R. Acquafredda, ${ }^{a}, 1$ T. Adam, ${ }^{b}$ N. Agafonova, ${ }^{c}$ P. Alvarez Sanchez, ${ }^{d}$ M. Ambrosio, ${ }^{a}$ A. Anokhina, ${ }^{e}$ S. Aoki, ${ }^{f}$ A. Ariga,${ }^{g}$ T. Ariga, ${ }^{h}$ L. Arrabito,${ }^{i}$ C. Aufranc, ${ }^{i}$ D. Autiero,${ }^{i}$ A. Badertscher,${ }^{j}$ A. Bagulya, ${ }^{k}$ E. Baussan, ${ }^{b}$ A. Bergnoli, ${ }^{l, 2}$ F. Bersani Greggio, ${ }^{m}$ A. Bertolin, ${ }^{l}$ M. Besnier, ${ }^{n, 3}$ D. Biaré, ${ }^{n}$ D. Bick, ${ }^{o}$ S. Blin, ${ }^{p}$ K. Borer,${ }^{g}$ J. Boucrot,${ }^{p}$ D. Boutigny, ${ }^{n, 4}$ V. Boyarkin, ${ }^{c}$ C. Bozza, ${ }^{q}$ T. Brugière,${ }^{i}{ }^{2}$. Brugnera, ${ }^{r, l}$ G. Brunetti,${ }^{s, t}$ S. Buontempo, ${ }^{a}$ J.E. Campagne,${ }^{p}$ B. Carlus,${ }^{i}$ E. Carrara $,{ }^{r l}, 5$ A. Cazes, ${ }^{l}$

L. Chaussard, ${ }^{i}$ M. Chernyavsky, ${ }^{k}$ V. Chiarella, ${ }^{i}$ N. Chon-Sen, ${ }^{b}$ A. Chukanov, ${ }^{a}$ R. Ciesielski, ${ }^{l}$ L. Consiglio, ${ }^{s, t}$ M. Cozzi, ${ }^{s}$ G. D'Amato, ${ }^{q}$ F. Dal Corso, ${ }^{l}$ N. D’Ambrosio, ${ }^{\nu}$ J. Damet,${ }^{n, 6}$ C. de La Taille, ${ }^{p}$ G. De Lellis, ${ }^{w, a, 7}$ Y. Déclais, ${ }^{i}$ T. Descombes, ${ }^{i, 8}$ M. De Serio, ${ }^{x}$ F. Di Capua, ${ }^{a}$ D. Di Ferdinando, ${ }^{t}$ A. Di Giovanni, ${ }^{y}$ N. Di Marco, ${ }^{y}$ C. Di Troia, ${ }^{u}$ N. Dick, ${ }^{b}$ S. Dmitrievski, ${ }^{z}$ A. Dominjon, ${ }^{i}$ M. Dracos, ${ }^{b}$ D. Duchesneau, ${ }^{n}$ B. Dulach, ${ }^{u}$ S. Dusini, ${ }^{l}$ J. Ebert, ${ }^{o}$ I. Efthymiopoulos, ${ }^{d}$ O. Egorov, ${ }^{a}{ }^{a}$ K. Elsener, ${ }^{d}$ R. Enikeev, ${ }^{c}$ A. Ereditato,${ }^{g}$ L. S. Esposito, ${ }^{\nu}$ C. Fanin, ${ }^{l}$ J. Favier, ${ }^{n}$ G. Felici, ${ }^{u}$ T. Ferber ${ }^{o}{ }^{o}$ R. Fini, ${ }^{x}$ L. Fournier,${ }^{n}$ A. Franceschi, ${ }^{u}$ D. Frekers ${ }^{a b}$ T. Fukuda, ${ }^{h}$ C. Fukushima, ${ }^{a c}$ V.I. Galkin, ${ }^{e}$ V.A. Galkin, ${ }^{a d}$ R. Gallet, ${ }^{n}$ S. Gardien, ${ }^{i}$ A. Garfagnini, ${ }^{r l}$ G. Gaudiot, ${ }^{b}$ G. Giacomelli, ${ }^{s, t}$ M. Giorgini, ${ }^{s, t}$ C. Girerd, ${ }^{i}$ C. Goellnitz, ${ }^{o}$ T. Goeltzenlichter, ${ }^{b}$ J. Goldberg, ${ }^{a e}$ D. Golubkov, ${ }^{a}{ }^{a}$ Y. Gornushkin, ${ }^{z}$ J.-N. Grapton, ${ }^{b}$ G. Grella, ${ }^{q}$ F. Grianti, ${ }^{m}$ E. Gschwendtner,${ }^{d}$ C. Guerin, ${ }^{i}$ M. Guler, ${ }^{a f}$ C. Gustavino, ${ }^{,}$ J.-L. Guyonnet, ${ }^{b}$ C. Hagner ${ }^{o}{ }^{\circ}$. Hamane, ${ }^{n, 9}$ T. Hara, ${ }^{f}$ M. Hauger,${ }^{g}$ M. Hess, ${ }^{g}$ M. Hierholzer, ${ }^{a g}$ K. Hoshino, ${ }^{h}$ M. leva, ${ }^{x}$ M. Incurvati, ${ }^{u}$ K. Jakovcic,${ }^{a h}$ J. Janicsko Csathy, ${ }^{g}$ B. Janutta, ${ }^{o}$ C. Jollet, ${ }^{b}$ F. Juget, ${ }^{g}$ M. Kazuyama, ${ }^{h}$ S.H. Kim, ${ }^{a i, 10}$ N. Khovansky, ${ }^{z}$ M. Kimura, ${ }^{a c}$ B. Klicek, ${ }^{a h}$ J. Knuesel, ${ }^{g}$ K. Kodama, ${ }^{a j}$ D. Kolev, ${ }^{a k}$

\footnotetext{
${ }^{1}$ Now at a private company

${ }^{2}$ Now at a private company

${ }^{3}$ Now at Laboratoire Leprince-Ringuet - École polytechnique, 91128 Palaiseau Cedex (France)

${ }^{4}$ Now at Centre de Calcul IN2P3/CNRS, 27 boulevard du 11 Novembre 1918, 69622 Villeurbanne Cedex (France)

${ }^{5}$ Now at a private company

${ }^{6}$ Now at Institut Universitaire de Radiophysique Appliquée, Grand Pré 1, CH-1007 Lausanne (Suisse)

${ }^{7}$ Supported by travel fellowship from the School of Sciences and Technology - University of Naples Federico II

${ }^{8}$ Now at CNRS, Station Biologique de Roscoff, Place Georges Teissier BP74, 29682 Roscoff Cedex

${ }^{9}$ Now at Lyonnaise des Eaux de Casablanca, 48 rue Mohamed Diouri, 20000 Casablanca (Morocco)

${ }^{10}$ Now at Chonnam National University (Korea)
} 
M. Komatsu, ${ }^{h}$ U. Kose, ${ }^{a f, 11}$ A. Krasnoperov, ${ }^{z}$ I. Kreslo, ${ }^{g}$ Z. Krumstein, ${ }^{z}$ V.V. Kutsenov, ${ }^{c}$ V.A. Kuznetsov, ${ }^{c}$ I. Laktineh, ${ }^{i}$ M. Lavy, ${ }^{n, 12}$ C. Lazzaro, ${ }^{j}$ T.D. Le, ${ }^{b}$

T. Le Flour, ${ }^{n}$ J. Lenkeit, ${ }^{o}$ J. Lewis, ${ }^{d}$ S. Lieunard, ${ }^{n}$ A. Ljubicic ${ }^{a h}$ A. Longhin, ${ }^{r}$

G. Lutter, ${ }^{g}$ A. Malgin,${ }^{c}$ K. Manai, ${ }^{i}$ G. Mandrioli, ${ }^{t}$ A. Marotta, ${ }^{a}$ J. Marteau, ${ }^{i}$

G. Martin-Chassard,,$^{p}$ V. Matveev, ${ }^{c}$ N. Mauri,,$^{s, t}$ M. Meddahi, ${ }^{d}$ F. Meisel, ${ }^{g}$

A. Meregaglia, ${ }^{b}$ A. Meschini, ${ }^{v}$ M. Messina, ${ }^{g}$ P. Migliozzi,${ }^{a}$ P. Monacelli, ${ }^{y}$ I. Monteiro, ${ }^{n}$

F. Moreau, ${ }^{n, 13}$ K. Morishima, ${ }^{h}$ U. Moser, ${ }^{g}$ M.T. Muciaccia, ${ }^{a l, x}$ P. Mugnier, ${ }^{n}$

N. Naganawa, ${ }^{h}$ M. Nakamura, ${ }^{h}$ T. Nakano, ${ }^{h}$ T. Napolitano, ${ }^{h}$ V. Nikitina,${ }^{e}$ K. Niwa, ${ }^{h}$

Y. Nonoyama, ${ }^{h}$ A. Nozdrin, ${ }^{z}$ S. Ogawa, ${ }^{c}$ A. Olchevski, ${ }^{z}$ D. Orlandi, ${ }^{,}$G. Orlova, ${ }^{k}$

V. Osedlo, ${ }^{e}$ D. Ossetski, ${ }^{a d}$ M. Paniccia, ${ }^{a}$ A. Paoloni, ${ }^{u}$ B. D Park, ${ }^{h}$ I.G. Park, ${ }^{a i}$

A. Pastore $,{ }^{a l}, x$ L. Patrizii, ${ }^{t}$ L. Pellegrino, ${ }^{h}$ E. Pennacchio, ${ }^{i}$ H. Pessard,${ }^{n}$ V. Pilipenko, ${ }^{a b}$

C. Pistillo, ${ }^{g}$ N. Polukhina, ${ }^{k}$ M. Pozzato, ${ }^{s, t}$ K. Pretzl,,${ }^{g}$ P. Publichenko, ${ }^{e}$ F. Pupilli, ${ }^{y}$

L. Raux,${ }^{p}$ J.P. Repellin, ${ }^{p}$ R. Rescigno, ${ }^{q}$ D. Rizhikov, ${ }^{a d}$ T. Roganova, ${ }^{e}$ G. Romano, ${ }^{q}$

G. Rosa, ${ }^{a m}$ I. Rostovtseva, ${ }^{a}{ }^{a}$ A. Rubbia,${ }^{j}$ A. Russo, ${ }^{w, a}$ V. Ryasny, ${ }^{c}$ O. Ryazhskaya,${ }^{c}$

A. Sadovski, ${ }^{z}$ C. Sanelli, ${ }^{l}$ O. Sato, ${ }^{h}$ Y. Sato, ${ }^{a n}$ V. Saveliev, ${ }^{a d}$ G. Sazhina, ${ }^{e}$

A. Schembri, ${ }^{a m}$ W. Schmidt Parzefall, ${ }^{o}$ H. Schroeder,${ }^{a g}$ H.U. Schütz,${ }^{g}$ J. Schuler, ${ }^{b}$

L. Scotto Lavina, ${ }^{a}$ J. Serrano, ${ }^{d}$ H. Shibuya, ${ }^{a c}$ S. Simone,,${ }^{a l, x}$ M. Sioli,,${ }^{s, t}$ C. Sirignano, ${ }^{q}$

G. Sirri, ${ }^{t}$ J.S. Song,${ }^{a i}$ M. Spinetti, ${ }^{u}$ L. Stanco,${ }^{r}$ N. Starkov,${ }^{k}$ M. Stipcevic, ${ }^{a h}$

T. Strauss, ${ }^{j}$ P. Strolin, ${ }^{w, a}$ V. Sugonyaev, ${ }^{r, 14}$ S. Takahashi, ${ }^{h}$ V. Talochkin,,${ }^{c}$ M. Tenti, ${ }^{s, t}$

V. Tereschenko, ${ }^{z}$ F. Terranova,,${ }^{u 15}$ I. Tezuka, ${ }^{a n}$ V. Tioukov, ${ }^{a}$ P. Tolun, ${ }^{a f}$ V. Tsarev, ${ }^{k}$

R. Tsenov, ${ }^{a k}$ S. Tufanli, ${ }^{a f}$ U. Ugolino, ${ }^{a}$ N. Ushida, ${ }^{a j}$ G. Van Beek, ${ }^{a o}$ V. Verguilov, ${ }^{a k}$

T. Viant, ${ }^{j}$ P. Vilain, ${ }^{a o}$ M. Vladimirov, ${ }^{k}$ L. Votano, ${ }^{u}$ J.L. Vuilleumier, ${ }^{g}$ T. Waelchli, ${ }^{g}$

M. Weber, ${ }^{g}$ G. Wilquet, ${ }^{a o}$ B. Wonsak, ${ }^{o}$ J. Wurtz, ${ }^{b}$ V. Yakushev, ${ }^{c}$ C.S. Yoon, ${ }^{a i}$

Y. Zaitsev, ${ }^{a}{ }^{a}$ A. Zghiche $^{n}$ and R. Zimmermann ${ }^{\circ}$

${ }^{a}$ INFN Sezione di Napoli, 80125 Napoli, Italy

${ }^{b}$ IPHC, Université de Strasbourg, CNRS/IN2P3, F-67037 Strasbourg, France

${ }^{c}$ INR-Institute for Nuclear Research of the Russian Academy of Sciences, 117312 Moscow, Russia

${ }^{d}$ CERN, European Organization for Nuclear Research, CH-1211 Geneva 23, Switzerland

e SINP MSU-Skobeltsyn Institute of Nuclear Physics of Moscow State University, 119992 Moscow, Russia

${ }^{f}$ Kobe University, 657-8501 Kobe, Japan

${ }^{g}$ Centre for Research and Education in Fundamental Physics, Laboratory for High Energy Physics (LHEP), University of Bern, $\mathrm{CH}-3012$ Bern, Switzerland

${ }^{h}$ Nagoya University, 464-8602 Nagoya, Japan

${ }^{i} I P N L$, Université Claude Bernard Lyon 1, CNRS/IN2P3, 69622 Villeurbanne, France

${ }^{j}$ ETH Zurich, Institute for Particle Physics, 8093 Zurich, Switzerland

${ }^{k}$ LPI-Lebedev Physical Institute of the Russian Academy of Sciences, 117924 Moscow, Russia

${ }^{l}$ INFN Sezione di Padova, 35131 Padova, Italy

\footnotetext{
${ }^{11}$ Supported by the International Postdoctoral Research Scholarship Programme from "The Scientific and Technological Research Council of Turkey", TUBITAK, Turkey.

${ }^{12}$ Now at chemin Combe Langin, 74890 Bons en Chablais (France)

${ }^{13}$ Now at Institut de Neurosciences Cognitives de la Méditerranée - CNRS, 31 chemin Joseph Aiguier, 13402 Marseille Cedex 20 (France)

${ }^{14}$ Now at IHEP, Protvino, Russia

${ }^{15}$ Corresponding author.
} 
${ }^{m}$ CSAAE - Urbino University and INFN - Laboratori Nazionali di Frascati, Italy

${ }^{n}$ LAPP, Université de Savoie, CNRS/IN2P3, 74941 Annecy-le-Vieux, France

${ }^{o}$ Hamburg University, 22761 Hamburg, Germany

${ }^{p}$ LAL, Université de Paris-Sud 11, CNRS/IN2P3, F- 91898 Orsay, France

${ }^{q}$ Dipartimento di Fisica dell'Università di Salerno and INFN, 84084 Fisciano, Salerno, Italy

${ }^{r}$ Dipartimento di Fisica dell'Università di Padova, 35131 Padova, Italy

${ }^{s}$ Dipartimento di Fisica dell'Università di Bologna, 40127 Bologna, Italy

${ }^{t}$ INFN Sezione di Bologna, 40127 Bologna, Italy

${ }^{u}$ Laboratori Nazionali di Frascati dell'INFN, 00044 Frascati (Roma), Italy

${ }^{v}$ INFN - Laboratori Nazionali del Gran Sasso, I-67010 Assergi (L'Aquila), Italy

${ }^{w}$ Dipartimento di Fisica dell'Università Federico II di Napoli, 80125 Napoli, Italy

${ }^{x}$ INFN Sezione di Bari, 70126 Bari, Italy

${ }^{y}$ Dipartimento di Fisica dell'Università dell'Aquila and INFN, I-67100 L'Aquila, Italy

${ }^{z}$ JINR-Joint Institute for Nuclear Research, 141980 Dubna, Russia

${ }^{a a}$ ITEP-Institute for Theoretical and Experimental Physics, 117259 Moscow, Russia

${ }^{a b}$ University of Münster, 48149 Münster, Germany

${ }^{a c}$ Toho University, 274-8510 Funabashi, Japan

ad Obninsk State University, Institute of Nuclear Power Engineering, 249020 Obninsk, Russia

${ }^{a e}$ Department of Physics, Technion, 32000 Haifa, Israel

af METU-Middle East Technical University, TR-06531 Ankara, Turkey

${ }^{a g}$ Fachbereich Physik der Universität Rostock, 18051 Rostock, Germany

${ }^{a h}$ IRB-Rudjer Boskovic Institute, 10002 Zagreb, Croatia

${ }^{a i}$ Gyeongsang National University, 900 Gazwa-dong, Jinju 660-300, Korea

${ }^{a j}$ Aichi University of Education, 448-8542 Kariya (Aichi-Ken), Japan

${ }^{a k}$ Faculty of Physics, Sofia University "St. Kliment Ohridski”, 1000 Sofia, Bulgaria

${ }^{a l}$ Dipartimento di Fisica dell'Università di Bari, 70126 Bari, Italy

${ }^{a m}$ Dipartimento di Fisica dell'Università di Roma "La Sapienza” and INFN, 00185 Roma, Italy

${ }^{a n}$ Utsunomiya University, 321-8505 Tochigi-Ken, Utsunomiya, Japan

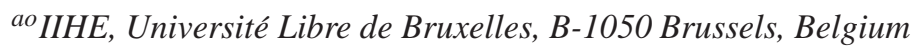

E-mail: francesco.terranova@cern.ch

ABSTRACT: The OPERA neutrino oscillation experiment has been designed to prove the appearance of $v_{\tau}$ in a nearly pure $v_{\mu}$ beam (CNGS) produced at CERN and detected in the underground Hall C of the Gran Sasso Laboratory, $730 \mathrm{~km}$ away from the source. In OPERA, $\tau$ leptons resulting from the interaction of $v_{\tau}$ are produced in target units called bricks made of nuclear emulsion films interleaved with lead plates. The OPERA target contains 150000 of such bricks, for a total mass of $1.25 \mathrm{kton}$, arranged into walls interleaved with plastic scintillator strips. The detector is split into two identical supermodules, each supermodule containing a target section followed by a magnetic spectrometer for momentum and charge measurement of penetrating particles. Real time information from the scintillators and the spectrometers provide the identification of the bricks where the neutrino interactions occurred. The candidate bricks are extracted from the walls and, after X-ray marking and an exposure to cosmic rays for alignment, their emulsion films are developed and sent to the emulsion scanning laboratories to perform the accurate scan of the event. In this paper, we 
review the design and construction of the detector and of its related infrastructures, and report on some technical performances of the various components. The construction of the detector started in 2003 and it was completed in Summer 2008. The experiment is presently in the data taking phase. The whole sequence of operations has proven to be successful, from triggering to brick selection, development, scanning and event analysis.

KEYWORDS: Large detector systems for particle and astroparticle physics; Particle tracking detectors; Spectrometers; Hybrid detectors 


\section{Contents}

1 Introduction $\quad 2$

2 The CNGS beam facility 5

3 OPERA lead/emulsion bricks $\quad 10$

$\begin{array}{ll}3.1 & \text { Nuclear emulsion films } \\ \end{array}$

\begin{tabular}{ll}
3.2 & Bricks and Changeable Sheets \\
\hline
\end{tabular}

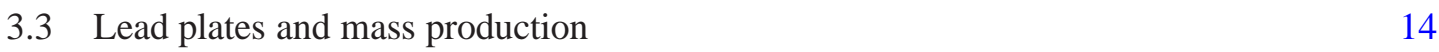

3.4 Brick Assembly Machine 16

4 The instrumented target $\quad 17$

$\begin{array}{ll}\text { 4.1 Wall mechanical structure } & 17\end{array}$

$\begin{array}{ll}4.2 & \text { Brick Manipulator System } \\ 4.3 & \text { Scintillator Target Trackers }\end{array}$

4.3 Scintillator Target Trackers 23

$\begin{array}{lll}4.4 & \text { Veto system } & 25\end{array}$

5 Magnetic spectrometers $\quad 27$

$\begin{array}{lll}5.1 & \text { Magnets } & 27\end{array}$

5.2 RPC/XPC tracking system 28

5.3 Precision trackers 32

6 Detector alignment 36

7 Data acquisition and software 37

$\begin{array}{lll}\text { 7.1 Global DAQ architecture } & 38\end{array}$

7.2 Event building and online software 40

7.3 UTC time synchronization 40

7.4 Offline software 41

8 Emulsion handling and analysis $\quad \mathbf{4 2}$

8.1 Brick extraction and film development 42

$\begin{array}{ll}8.2 & \text { Emulsion scanning systems }\end{array}$

9 Emulsion scanning methods 46

10 Conclusions $\quad 50$ 


\section{Introduction}

There is today convincing evidence from the observed oscillation of atmospheric, solar and terrestrial neutrinos that neutrino mass and flavor eigenstates do not coincide, and that their mass eigenvalues are not equal [1-4]. At adequate distance from the source, neutrinos produced in a specific flavor state may therefore be detected as a different flavor state. The appearance of a neutrino flavor different from the original one is the cleanest signature of oscillations. Nevertheless, so far, the oscillation has been mainly studied through disappearance of the original flavor or from the ratio between the (flavor sensitive) charged-currents and the (flavor blind) neutral-current interactions. Indeed, solar neutrinos have not enough energy to produce charged leptons other than electrons while, at the atmospheric neutrino scale, the leading oscillation channel is $v_{\mu} \rightarrow v_{\tau}$. The latter requires a detector capable of identifying the short-lived and high-mass $\tau$ lepton.

The OPERA experiment [5-7] is designed for the first direct observation of $v_{\tau}$ appearance from $v_{\mu} \rightarrow v_{\tau}$ oscillation in the CNGS [8] long baseline beam from CERN to the underground Gran Sasso Laboratory (LNGS), $730 \mathrm{~km}$ away, where the OPERA detector is located. Having the capability to observe prompt electrons, OPERA will also allow the search for the sub-leading oscillation channel $v_{\mu} \rightarrow v_{e}$.

The CNGS ("CERN Neutrinos to Gran Sasso") beam - described in section 2 - consists mainly of $v_{\mu}$ with a mean energy of about $17 \mathrm{GeV}$, with a $\bar{v}_{\mu} \mathrm{CC}$ event contamination of $2.1 \%$ and an electron (anti-)neutrino contamination of $\sim 0.9 \%$. The high energy of the beam (above the threshold for $\tau$ lepton production) and the fact that the $v_{\tau}$ prompt contamination is totally negligible make the beam suitable for the study of $v_{\mu} \rightarrow v_{\tau}$ transitions in appearance mode. The beam energy was optimized to maximize the $\tau$ rate at the detector site and results from a compromise between two opposite requirements: a significant charged-current interaction cross-section of the oscillated $v_{\tau}$, which occurs at high energy values, and a large oscillation probability favoring low energies.

OPERA was specifically designed to identify the $\tau$ via the topological observation of its decay, followed by a kinematical analysis of the event. This is done by means of a hybrid apparatus that combines real-time detection techniques ("electronic detectors") and the Emulsion Cloud Chamber (ECC) technique [9]. An ECC detector is made of passive material plates, used as target, alternated with nuclear emulsion films used as tracking devices with sub-micrometric accuracy.

The ECC technique has a long history, being adopted soon after the Second World War in experiments on cosmic-rays and leading to the first observation of charmed particles in 1971 [10], three years before its observation in accelerator experiments. At that time, an emulsion experiment was almost entirely based on the visual inspection of the films by experienced operators and it was close to be abandoned when the development of fully automated microscope scanning systems brought this detector to a revival in the early nineties. The DONUT experiment, which has first observed the $v_{\tau}$ [11] is a successful application of the ECC technology at a mass scale of $100 \mathrm{~kg}$.

The sub-micrometric position accuracy and the use of passive material allow for momentum measurement of charged particles by the detection of multiple Coulomb scattering, identification and measurement of electromagnetic showers and electron/pion separation. Such studies have been conducted during the last years in the framework of the OPERA experiment [12-15]. The ECC has proven the unique feature of combining a tracker of an extremely high accuracy with the capability of performing kinematical variable measurements. 
Today OPERA brings the ECC technology to a much larger scale: the basic unit of the experiment is a "brick" made of 56 plates of lead ( $1 \mathrm{~mm}$ thick) interleaved with nuclear emulsion films (industrially produced) for a total weight of $8.3 \mathrm{~kg}$. 150000 of such target units have been assembled to reach an overall mass of $1.25 \mathrm{kton}$. The bricks are arranged in 62 vertical "walls", transverse to the beam direction, interleaved with planes of plastic scintillators. The detector is subdivided in two identical units (supermodules), each consisting of 31 walls and 31 double layers of scintillator planes followed by a magnetic spectrometer (see figure 2).

The electronic detectors have the task to trigger the data acquisition, identify and measure the trajectory of charged particles and locate the brick where the interaction occurred. The momentum of muons is measured by the spectrometers while their trajectories are traced back through the scintillator planes up to the brick where the track originates. When no muons are observed, the scintillator signals produced by electrons or hadronic showers are used to predict the location of the brick that contains the primary neutrino interaction vertex, hence providing "time resolution" to the emulsions.

The selected brick is then extracted from the target and the two interface emulsion films, called "Changeable Sheets" (CS), attached on the downstream face of the brick are developed. If tracks related to the neutrino interaction are observed in these CS, the films of the brick are also developed and the tracks are followed back by fully automated scanning microscopes until the vertex is located. The analysis of the event topology at the primary vertex will then select possible $\tau$ candidates. The latter might include one track that shows a clear "kink" due to the decay-in-flight of the tau (long decays) or an anomalous impact parameter with respect to the primary vertex (short decays) compatible with a decay-in-flight in the first lead plate. They will then be confirmed by a kinematical analysis at the primary and decay vertices. Figure 1 shows a schematic view of a $v_{\tau}$ charged-current interaction with a long decay of the $\tau$ lepton as it would appear in an OPERA brick, in the CS and in the scintillator trackers.

The modular structure of the target allows to extract only the bricks actually hit by the neutrinos, thus achieving a quasi online analysis of the interaction and minimizing the target mass reduction during the run.

The structure of the OPERA detector is illustrated in figures 2 and 3. Each brick wall hosting 2912 bricks and supported by a light stainless steel structure is followed by a double layer of plastic scintillators (Target Trackers, TT) that provide real-time detection of the outgoing charged particles. The instrumented target is followed by a magnetic spectrometer consisting of a large iron magnet instrumented with plastic Resistive Plate Chambers (RPC). The deflection of charged particles inside the magnetized iron is measured by six stations of drift tubes (Precision Trackers, PT). Left-right ambiguities in the reconstruction of particle trajectories inside the PT are removed by means of additional RPC (XPC) with readout strips rotated by $\pm 45^{\circ}$ with respect to the horizontal and positioned near the first two PT stations. An instrumented target and its spectrometer is called a "supermodule" (SM). The whole OPERA detector is made of two such supermodules. Finally, two glass RPC planes mounted in front of the first target (VETO) allow to reject charged particles originating from outside the target fiducial region, coming from neutrino interactions in the surrounding rock material and in the Borexino experiment [16].

The installation of OPERA in the Hall C of LNGS started in Summer 2003 with the positioning of the two lower return yokes of the magnets. After completion of the first magnet (see section 5.1) 


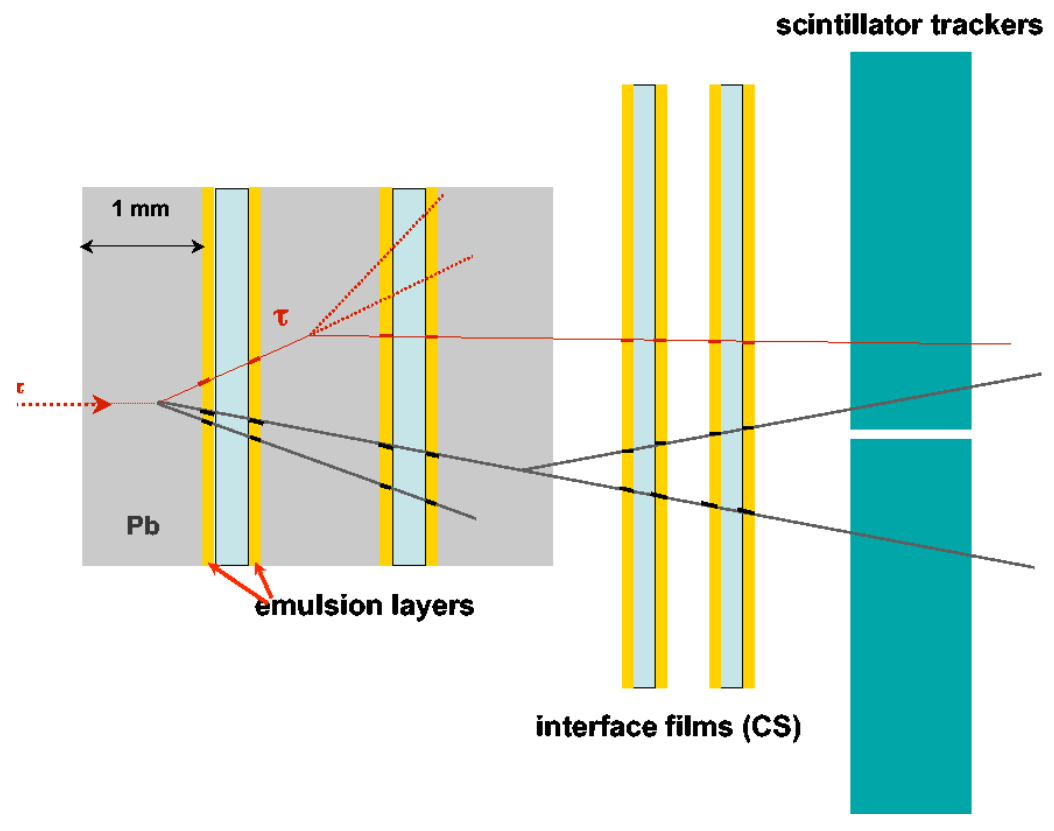

Figure 1. Schematic view of a $v_{\tau}$ charged-current interaction and the decay-in-flight of the final state $\tau$ lepton as it would appear in an OPERA brick, in the interface emulsion films (Changable Sheets - CS), and in the scintillator trackers (Target Trackers).

a support structure was installed in August 2004 to hold the first instrumented target. It consists of support rails for the walls, a top platform housing detector electronics and a seismic damping structure. The installation of the walls started in October 2004 and was completed in May 2006. At the time of the first CNGS pilot run (Summer 2006) all electronic detectors except the PT planes of SM2 and the VETO were operational. The PT and VETO installation was completed in 2007. Each wall and tracker plane has a surface of about $6.7 \times 6.7 \mathrm{~m}^{2}$, transverse to the beam direction. The overall dimension of the detector is $\sim 10 \times 10 \times 20 \mathrm{~m}^{3}$ for a total weight of about $4 \mathrm{kton}$.

During the mounting of the electronic detectors, emulsion films coming from Japan were progressively stored in a dedicated bunker in the LNGS Hall B. Massive brick production in the underground hall (to minimize the collection of cosmic-ray tracks) started after the installation of the Brick Manipulator System (BMS) and of the Brick Assembly Machine (BAM). Bricks produced by the BAM were stored in a cylindrical container (a "drum" containing 234 bricks). Thereafter, drums were transported to Hall $\mathrm{C}$ and were positioned on the loading station using a crane. The BMS robot extracted the bricks from the drum and inserted them into the walls. Brick mass production started in Spring 2007 and was completed recently.

OPERA is the first very large scale emulsion experiment. Just to give an idea, the 150000 ECC bricks include about 110,000 $\mathrm{m}^{2}$ emulsion films and 105,000 $\mathrm{m}^{2}$ lead plates. The scanning of the events is performed with more than 30 fully automated microscopes, each of them faster by about two orders of magnitude than those used e.g. in the CHORUS experiment [17]. In this paper, the CNGS beam and the overall OPERA detector will be described in detail. 


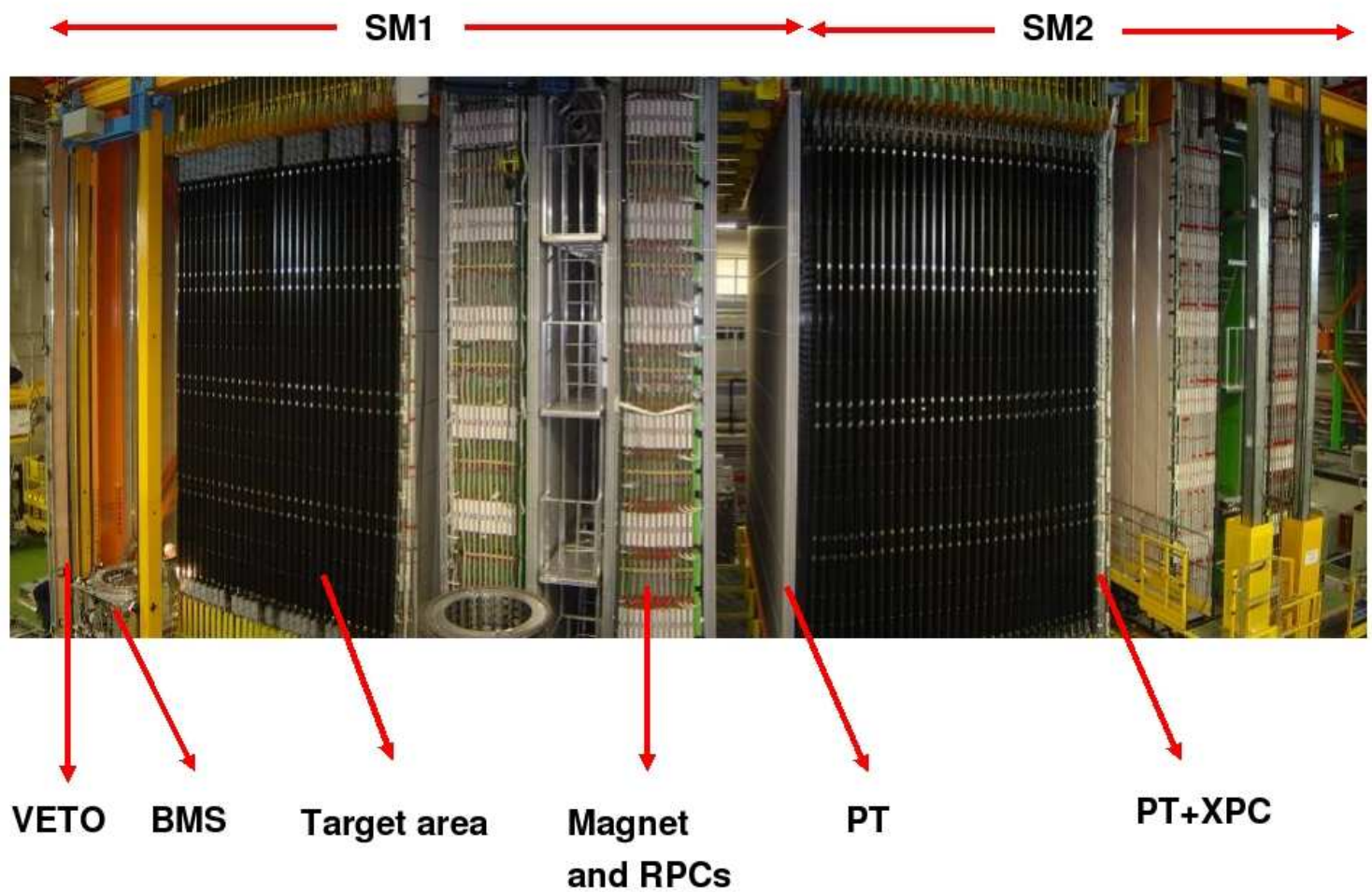

Figure 2. Fish-eye side view of the OPERA detector. The upper red horizontal lines indicate the position of the two identical supermodules (SM1 and SM2). The "target area" is made of walls filled with ECC bricks interleaved with planes of plastic scintillators (TT): the black covers are the end-caps of the TT. Arrows also show the position of the VETO planes, the drift tubes (PT) surrounded by the XPC, the magnets and the RPC installed between the magnet iron slabs. The Brick Manipulator System (BMS) is also visible.

\section{The CNGS beam facility}

The CNGS neutrino beam [18] is produced by a $400 \mathrm{GeV} / \mathrm{c}$ proton beam extracted from the SPS accelerator and transported along a $840 \mathrm{~m}$ long beam line onto a carbon target producing kaons and pions. The positively charged $\pi / \mathrm{K}$ are energy-selected and guided with two focusing lenses, called "horn" and "reflector", in the direction of Gran Sasso. These particles decay into $v_{\mu}$ and $\mu$ in a $1000 \mathrm{~m}$ long vacuum pipe. All the hadrons, i.e. protons that have not interacted in the target, pions and kaons that have not decayed in flight, are absorbed in a hadron stopper. Only neutrinos and muons are able to cross this $18 \mathrm{~m}$ long block of graphite and iron. The muons, which are ultimately absorbed downstream in around $500 \mathrm{~m}$ of rock, are monitored by two muon detector stations. This allows the measurement of the intensity of the neutrino beam produced and the beam profile.

The beam extracted from the SPS uses the same extraction channel as for one of the two LHC beams. After about $100 \mathrm{~m}$ from the extraction point, a string of switch magnets is used to direct the beam either to the LHC or to the CNGS target. The CNGS proton beam line consists of a $620 \mathrm{~m}$ long arc to bend the beam in the direction of Gran Sasso with a slope of 5.6\%, followed by a $120 \mathrm{~m}$ long focusing section to obtain the desired beam sizes on the target. The tunability of the optics allows varying the beam sizes on the target from $\sigma=0.25 \mathrm{~mm}$ to $1.0 \mathrm{~mm}$ [19]. During a nominal 


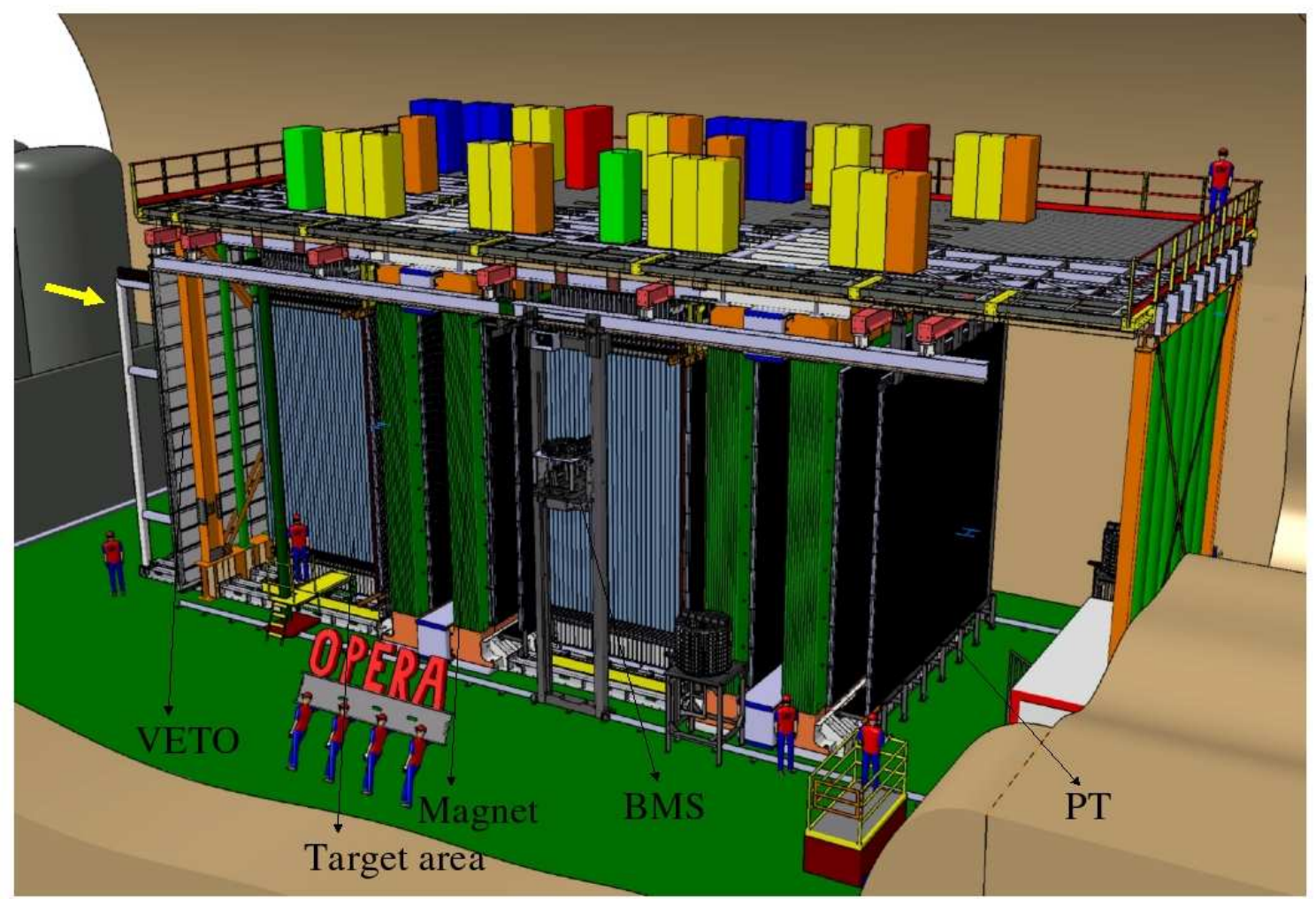

Figure 3. Artistic view of the OPERA detector. The yellow arrow in front of the VETO indicates the direction of the incoming CNGS neutrino beam.

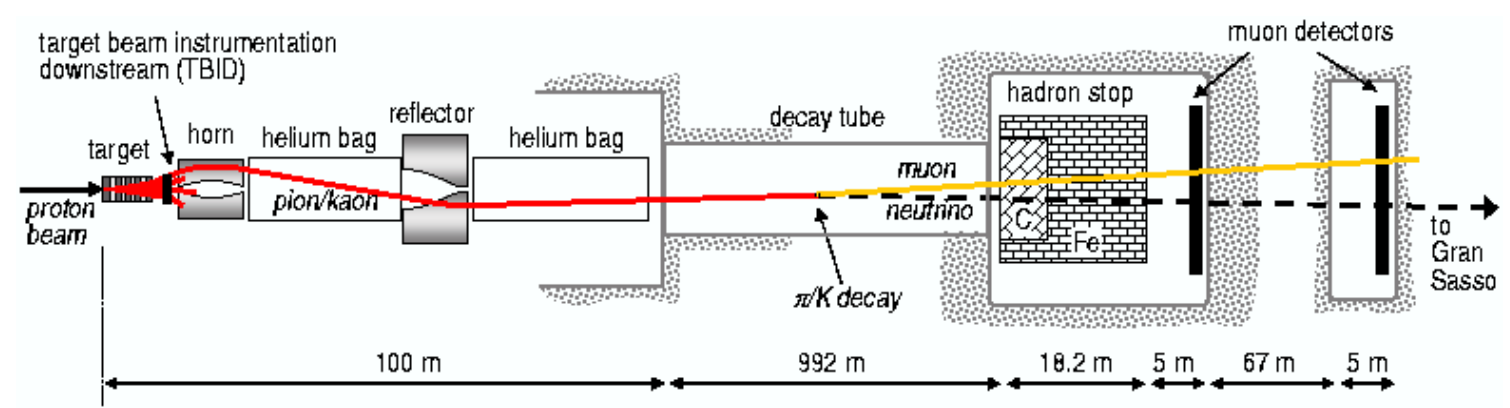

Figure 4. Main components of the CNGS beam line.

CNGS cycle, i.e. every $6 \mathrm{~s}$, there are two SPS extractions (10.5 $\mu \mathrm{s}$ each, separated by $50 \mathrm{~ms})$ of $2.410^{13}$ protons each at $400 \mathrm{GeV} / \mathrm{c}$. The nominal parameters for the CNGS proton beam are given in table 1 .

The magnetic system of the proton beam line is composed of 73 dipole magnets (nominal field of $1.7 \mathrm{~T}$ at $400 \mathrm{GeV} / \mathrm{c}$ [20]), 20 quadrupole magnets (nominal gradient of $40 \mathrm{~T} / \mathrm{m}$ [21]) and 12 corrector magnets (maximum deflection angle of $80 \mu \mathrm{rad}$ ) [22]. The 5.6\% slope of the proton beam line is provided by 32 horizontal dipole magnets tilted by $12.8^{\circ}$. The beam line can operate from $350 \mathrm{GeV} / \mathrm{c}$ (limited by the vertical aperture in the main bending magnets) to $450 \mathrm{GeV} / \mathrm{c}$ (maximum SPS energy). 
Table 1. CNGS beam parameters.

\begin{tabular}{|c|c|}
\hline Parameters & Nominal Values \\
\hline Normalized emittance $(\mu \mathrm{m})$ & $\mathrm{H}=12, \mathrm{~V}=7$ \\
Physical emittance $(\mathrm{nm})$ & $\mathrm{H}=28, \mathrm{~V}=16$ \\
Momentum spread & $0.07 \% \pm 20 \%$ \\
Number of extractions per cycle & $2(50 \mathrm{~ms}$ apart $)$ \\
Extraction batch length $(\mu \mathrm{s})$ & 10.5 \\
Number of bunches per extraction & 2100 \\
Intensity per extraction & $2.410^{13}$ \\
Bunch length $(\mathrm{ns})(4 \sigma)$ & 2 \\
Bunch spacing $(\mathrm{ns})$ & 5 \\
Beam beta at focus $(\mathrm{m})$ & $\mathrm{H}=10, \mathrm{~V}=20$ \\
Beam size at $400 \mathrm{GeV}(\mathrm{mm})(\sigma)$ & 0.5 \\
\hline
\end{tabular}

Beam monitors allow to track the high intensity proton beam and to verify its stability [23, 24]. The beam position is measured along the proton beam line using 23 Beam Position Monitors (BPM); all but the last one are button electrode monitors (the buttons were recuperated from the Large Electron-Positron Collider, LEP), and all are equipped with 2 plane read-out electronics. The last BPM is mechanically coupled to the target in order to provide the beam position as seen by the first target rod. It is an innovative strip-line coupler pick-up operated in air. While the tolerance requirement on the overall BPM is set to $\pm 0.6 \mathrm{~mm}$, this monitor is specified to operate with better than $\pm 0.35 \mathrm{~mm}$ tolerance. The 8 beam profile monitors are optical transition radiation monitors (OTR) using either a $75 \mu \mathrm{m} \mathrm{C}$ screen for high intensity operation or a $12 \mu \mathrm{m}$ Ti screen for low intensity beam. CCD cameras are used to observe the OTR radiation, a direct image of the proton beam crossing the screens. Two Beam Current Transformer monitors (BCT) are measuring the beam intensity at the start and the end of the line. 18 Beam Loss Monitors (BLM, $\mathrm{N}_{2}$ filled ionization chambers) measure the beam losses along the beam line.

The proton line is well tuned along its $840 \mathrm{~m}$ length and the beam stability in the transfer line and on the target is excellent [25]. The maximum beam excursion measured is well within the \pm $4 \mathrm{~mm}$ allowed by aperture constraints. The beam size along the proton beam line is very close to the simulated values; the beam spot at the target is measured to be $0.5 \mathrm{~mm}$ r.m.s, as expected. The beam position stability onto the target averaged over several days is $\sim 50 \mu \mathrm{m}$ r.m.s. [19].

The secondary beam line goes from the CNGS target to the muon monitors. A schematic overview of the CNGS secondary beam line at CERN is shown in figure 4.

The CNGS target (figure 5) is designed to withstand the beam induced stress (thermo mechanical shocks, heat deposit) up to $3.510^{13}$ protons per extraction with $400 \mathrm{GeV} / \mathrm{c}$ (up to 750 $\mathrm{kW}$ average power) [26]. The target magazine consists of five target units. One unit is used in the beam; the other four are in-situ spares. A target unit consists of an aluminum cylinder closed off with beryllium windows. Each target unit contains 13 graphite rods which are $10 \mathrm{~cm}$ long and interspaced by $9 \mathrm{~cm}$. The diameter of the first two rods is $5 \mathrm{~mm}$; the other rods have a diameter 


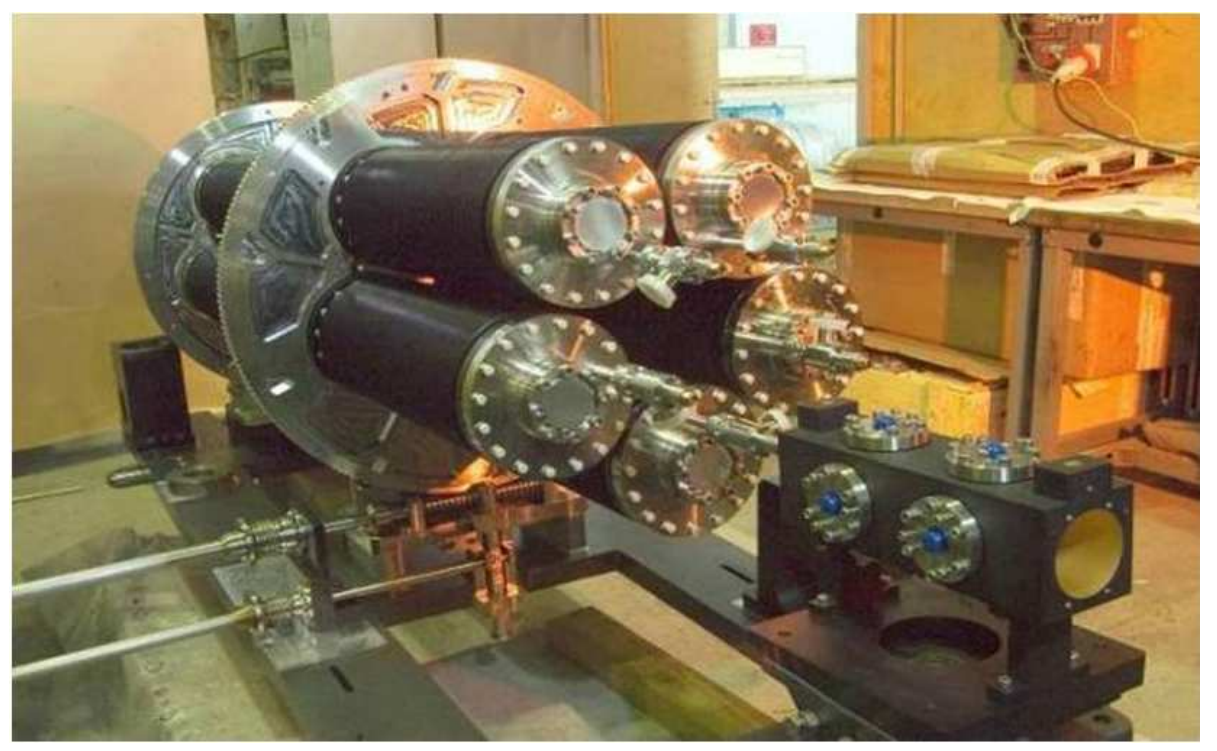

Figure 5. The CNGS target magazine consisting of five target units. The bottom unit intercepts the proton beam; the other four are in-situ spares.

of $4 \mathrm{~mm}$. The target magazine as well as the surrounding iron shielding are air-cooled. The target alignment to the proton beam is specified, at installation, to be $\pm 0.1 \mathrm{~mm}$ transversally.

The magnetic focusing system, comprising the horn and reflector (each $7 \mathrm{~m}$ long), is installed downstream of the target (see figure 6). The horn and reflector are toroidal lenses with a focusing magnetic field between the inner and outer conductor created by a pulsed high current. The shape of the inner conductor provides the desired focusing for particles with a wide range of momenta and angles. The horn is pulsed twice every cycle with a current of $150 \mathrm{kA}$ lasting few milliseconds, while the reflector is pulsed with $180 \mathrm{kA}$. The two pulses are separated by $50 \mathrm{~ms}$, synchronized with the two beam pulses. Polarity change of the magnetic lenses, e.g. required to study systematic effects, can be done remotely. The water cooling systems of the horn is designed for a proton intensity of $7.210^{13}$ protons per 6 s cycle $\left(3.610^{13}\right.$ protons/extraction) at $400 \mathrm{GeV} / \mathrm{c}$ [27]. The corresponding total power to be removed from the horn is $26 \mathrm{~kW}$, coming from the particle interactions in the horn material and the applied current.

Special features are implemented on the cavern crane and the CNGS equipment in order to replace the target or the horn remotely with spare ones in case of failure. Nevertheless, a limited number of manual interventions are usually needed before a remote exchange operation. Downstream of the horn there is a $31 \mathrm{~m}$ long tube with $1.2 \mathrm{~m}$ diameter filled with Helium at 20 mbar overpressure. A second Helium tube $41 \mathrm{~m}$ long is installed downstream of the reflector. At the end of the target cavern starts the CNGS decay tube. This steel tube is $18 \mathrm{~mm}$ thick and $994 \mathrm{~m}$ long, with a diameter of $2.45 \mathrm{~m}$. It is surrounded by $50 \mathrm{~cm}$ of concrete. The tube is under vacuum at less than 1 mbar. The entrance window is made of a $3 \mathrm{~mm}$ thick Titanium foil, the downstream window is a water cooled $50 \mathrm{~mm}$ carbon steel window. The water cooled hadron absorber, made of $3 \mathrm{~m}$ graphite and $15 \mathrm{~m}$ iron, is designed to absorb up to $100 \mathrm{~kW}$ of protons and hadrons.

Beam instrumentation in the secondary beam line is needed to measure the profile and intensity of pions and muons. Immediately downstream of the target, the efficiency with which protons 


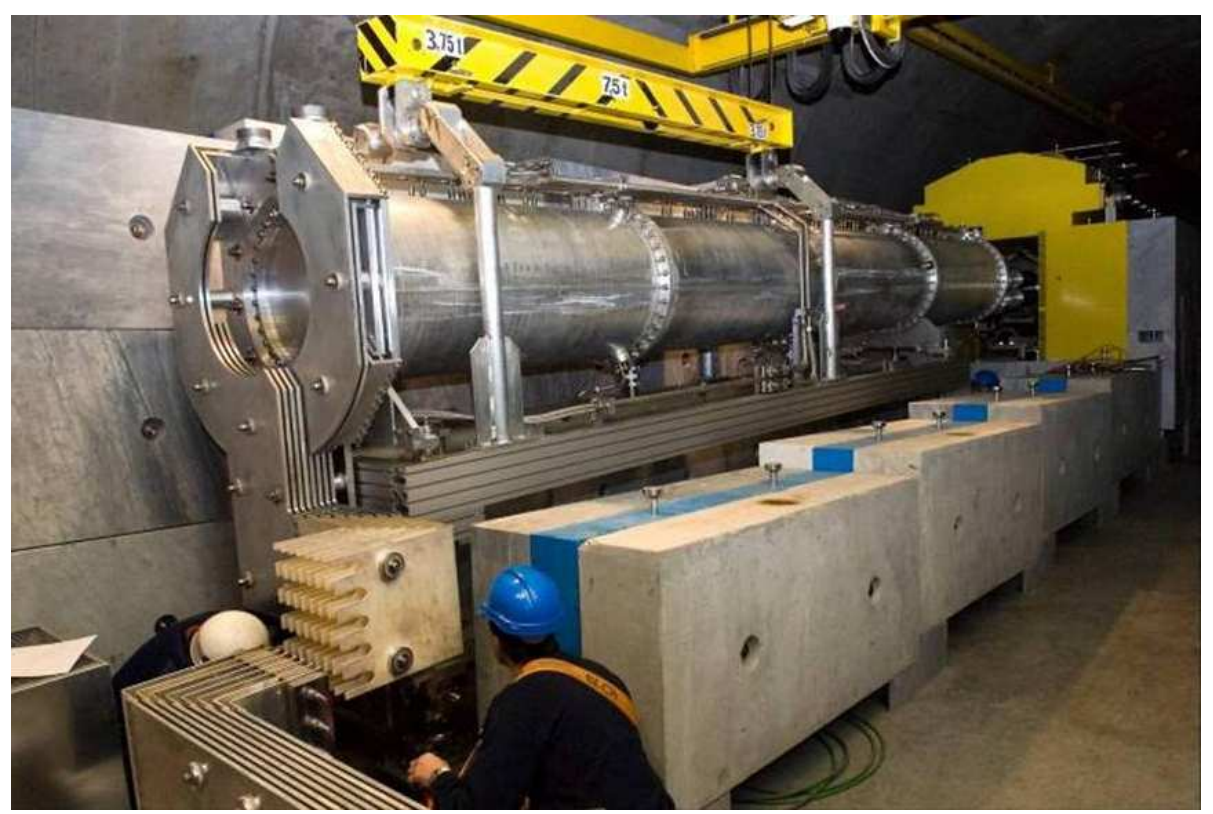

Figure 6. The CNGS horn during installation.

are converted into secondaries is checked with a Secondary Emission Monitor, made of $12 \mu \mathrm{m}$ thick titanium foils of $145 \mathrm{~mm}$ diameter mounted in a vacuum box $\left(<10^{-4}\right.$ mbar vacuum). A proton scan across the target provides information on the maximum production of charged particles, i.e. on the best alignment of the proton beam with respect to the target. As this detector is not expected to be operational for the full duration of the CNGS program due to the very high radiation environment, two ionization chambers (SPS-type beam loss monitors) are used as back-up. They are mounted at the same location downstream of the target, at the left and right of the mainstream and outside the shielding.

The CNGS facility has two muon detector stations (see figure 7) located downstream of the hadron stopper and separated by $67 \mathrm{~m}$ of rock. In each station, 42 muon detectors are installed to measure the muons created in association with the $v_{\mu}$ in the decay of the pions and kaons. The muon detectors are assembled in a cross-shaped array to provide the muon intensity and the vertical and horizontal muon profiles [28]. The distance between two neighbouring detectors is 11.25 $\mathrm{cm}$. The total height/width covered by the detectors is $2.7 \mathrm{~m}$. In addition, an identical monitor is installed on a motorized support, downstream of the fixed ones to allow cross-calibration of the fixed monitors and to probe the muon profile where there is no fixed monitor. The beam profiles from the muon stations provide on-line feedback for the quality control of the neutrino beam. The muon detectors must measure up to $10^{8}$ muons $/ \mathrm{cm}^{2}$ during one extraction, i.e. during 10.5 $\mu \mathrm{s}$. To cope with such a high instantaneous rate, nitrogen-filled sealed ionization chambers are used. These ionization chambers are also used as beam loss monitors (BLMs) at the Large Hadron Collider. Each $48 \mathrm{~cm}$ long detector has a diameter of $16 \mathrm{~cm}$, is filled with $\mathrm{N}_{2}$ at 1.1 bar and has 61 electrodes separated by $5 \mathrm{~mm}$. The bias electric field is set to $2400 \mathrm{~V} / \mathrm{cm}$ [29]. Since the chambers are separated by $67 \mathrm{~m}$ of rock, the muon energy spectrum is different in the two chambers. Only muons with an energy above $20 \mathrm{GeV}(50 \mathrm{GeV})$ reach the first (second) muon detector chamber. The 

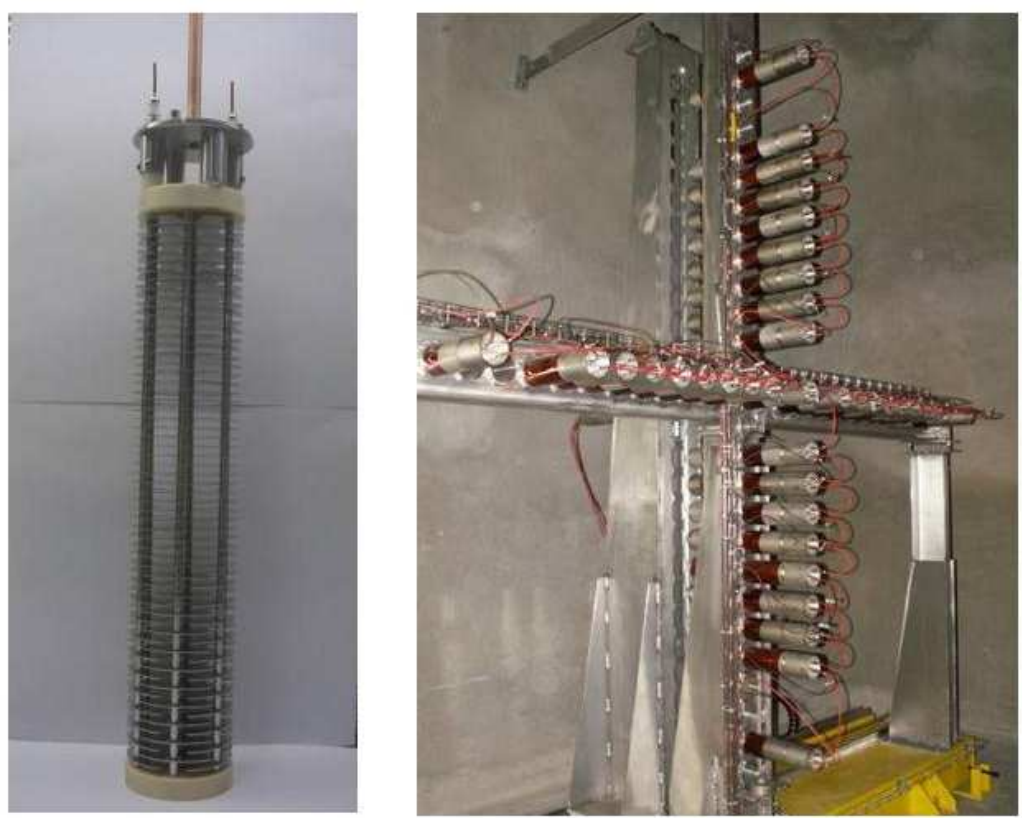

Figure 7. Left: a single muon detector without its stainless steel casing. Right: the muon detector cross-shaped array. The distance between two neighbouring ionization chambers is $11.25 \mathrm{~cm}$. The total height/width covered by the detector is $2.7 \mathrm{~m}$.

first muon detector station is very sensitive to misalignment between the target and the horn, while the second station gives information on proton-target alignment [30]. Hence scanning the proton beam w.r.t. the target and the horn and comparing the muon monitor profiles provides optimal secondary particle production efficiency and a precise alignment of the beam w.r.t the target and the horn [31]. The muon monitor reproducibility for all the detectors was measured to be better than $1 \%$ r.m.s. The response of the different muon monitors is identical within less than $1 \%$, as cross-checked with the motorized monitor [28].

In figure 8 , the flux of the four neutrino beam components is shown.

\section{OPERA lead/emulsion bricks}

\subsection{Nuclear emulsion films}

Nuclear emulsions consist of $\mathrm{AgBr}$ crystals scattered in a gelatine binder. The $\mathrm{AgBr}$ crystal, of $0.2 \mu \mathrm{m}$ diameter, is a semiconductor with a band gap of $2.6 \mathrm{eV}$. After the passage of a charged particle, electron-hole pairs are created in the crystal. The excited electrons are trapped in the lattice defects on the surface of the crystal and $\mathrm{Ag}$ metal atoms are created, which act as latent image centers. During a chemical-physical process known as development, the reducer in the developer gives electrons to the crystal through the latent image center and creates silver metal filaments using silver atoms from the crystal. This process multiplies the number of metal silver atoms by several orders of magnitude $\left(10^{8}-10^{10}\right)$ and the grains of silver atoms, of about $0.6 \mu \mathrm{m}$ diameter, become visible with an optical microscope. 

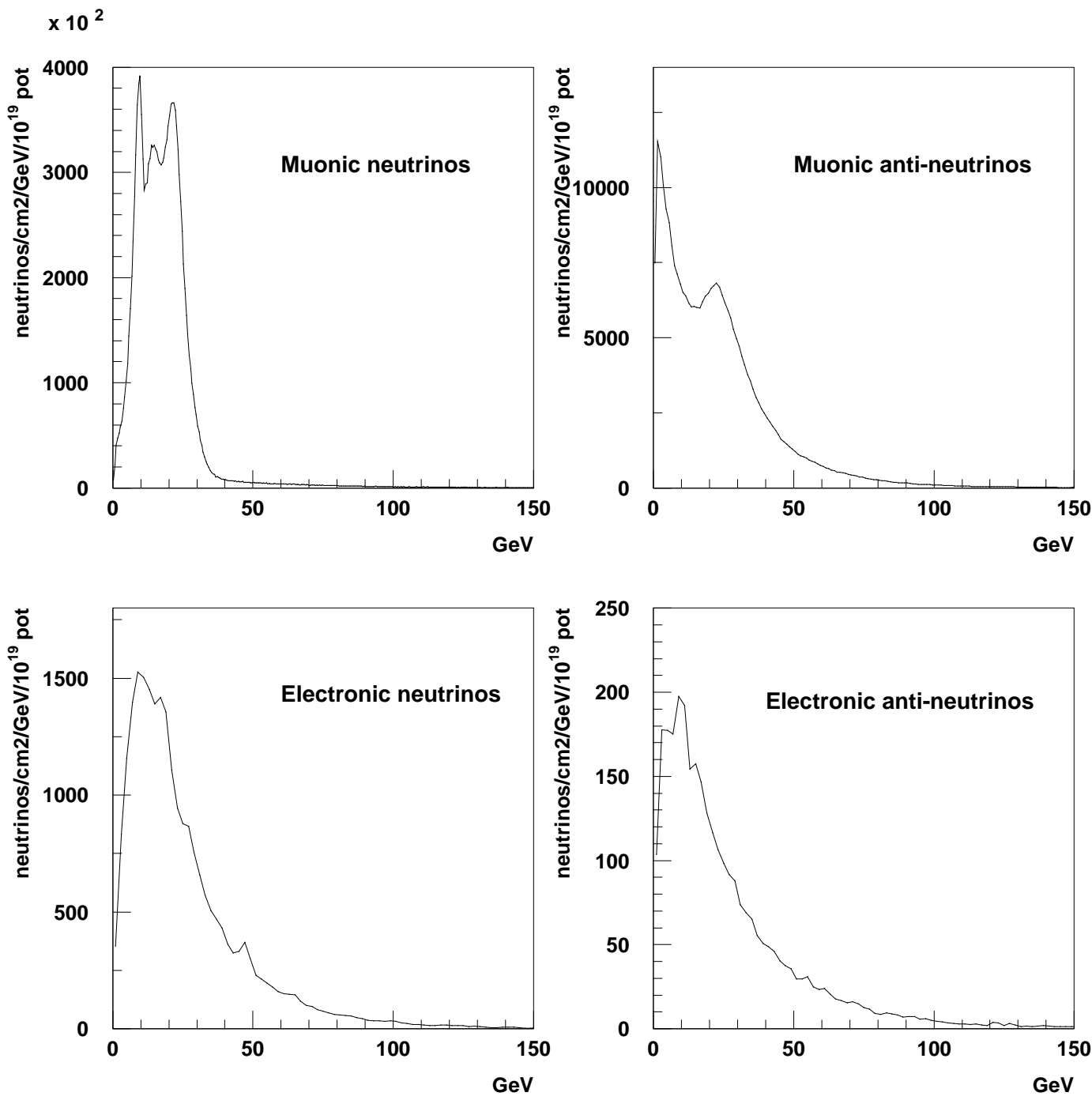

Figure 8. The four neutrino beam components of the CNGS.

The detection efficiency of a crystal is $\sim 0.16$ for minimum ionising particles and a charged particle penetrates $\sim 230$ crystals $/ 100 \mu \mathrm{m}$ along its path. Thus a minimum ionising particle leaves $\sim 36$ grains $/ 100 \mu \mathrm{m}$, which is the sensitivity of the film.

As already mentioned, the overall emulsion area in OPERA is larger than $100,000 \mathrm{~m}^{2}$. The pouring of such a large amount of nuclear emulsion gel could not be made by hand, as it was traditionally done for past experiments. For instance, the largest emulsion experiment so far, CHORUS [17], used $300 \mathrm{~m}^{2}$. Therefore, an industrial production was set-up after an R\&D program carried out in collaboration with the Fuji Film company. ${ }^{1}$ Details about the OPERA films are described in [32]. Here we report only the main features.

An OPERA film has 2 emulsion layers (each $44 \mu \mathrm{m}$ thick) on both sides of a transparent triacetylcellulose base ( $205 \mu \mathrm{m}$ thick). The total thickness is $293 \pm 5 \mu \mathrm{m}$. The transverse size is

\footnotetext{
${ }^{1}$ Fuji Photo Film Co., Ltd, Minamiashigara, Kanagawa 250-0193, Japan.
} 
$124.6 \pm 0.3 \mathrm{~mm} \times 99.0 \pm 0.2 \mathrm{~mm}$. The gel was derived from the ET7C type used for the DONUT experiment [11]. The intrinsic position resolution of this gel with OPERA normal developer is about $50 \mathrm{~nm}$, which corresponds to $0.35 \mathrm{mrad}$ as intrinsic angular resolution from the track positions at the two faces of the plastic base. The density of the gel is $2.7 \mathrm{~g} / \mathrm{cm}^{3}$ and the radiation length $\mathrm{X}_{0}$ is $5.0 \mathrm{~cm}$.

Nuclear emulsions integrate all tracks (cosmic rays, environmental radiations) from their production to their development. Since the full production process of a film set-up by the Fuji Film industrial plant takes about one month, each film integrates about 3,000 tracks $/ \mathrm{cm}^{2}$ from the cosmic ray flux, a value much larger than the maximum density of about $100 \mathrm{tracks} / \mathrm{cm}^{2}$ allowed for brick analysis (see section 8.2). In order to reduce this background, a new procedure called refreshing was set up. It consists in keeping the emulsion films at high relative humidity (RH) and high temperature (about $27^{\circ} \mathrm{C}$ or more). For example, at $98 \% \mathrm{RH}$ and $27^{\circ} \mathrm{C}$ for 3 days, the grain density of tracks accumulated before the refreshing goes from 36 to less than 10 grains $/ 100 \mu \mathrm{m}$, without affecting the sensitivity to tracks detected later on (34 grains $/ 100 \mu \mathrm{m}$ ).

To process about 10,000,000 films, a refresh facility was constructed in the Tono mine in Gifu, Japan. Two setups were constructed underground at $50 \mathrm{~m}$ and $100 \mathrm{~m}$, where the cosmic ray flux is $1 / 50$ and $1 / 400$ of the flux at the surface, respectively. The facility operates at $27^{\circ} \mathrm{C}$ and consists of 26 refreshing chambers, each capable of processing 8000 films per cycle. One cycle lasts five days: one day of open air humidification $(90 \% \mathrm{RH})$, three days of closed air humidification (98\% RH) and one day of dry mode $(40 \% \mathrm{RH})$. This process reduces the integrated number of tracks from $\sim 3000$ to less than 100 tracks $/ \mathrm{cm}^{2}$.

The randomly distributed grains (fog) induced by thermal excitation constitute a background for tracking. This background level is expressed by the number of grains per $1000 \mu \mathrm{m}^{3}$. The fog density is slightly increased by refreshing if the temperature does not exceed $30^{\circ} \mathrm{C}$. Measurements show a fog density of 3 and 6 grains per $1000 \mu \mathrm{m}^{3}$, respectively before and after the refreshing. The measurements provided by acceleration tests ${ }^{2}$ showed that the grain density would be 34 grains per $100 \mu \mathrm{m}$ and the fog density $14 \pm 1$ grains/1000 $\mu \mathrm{m}^{3}$ after 5 years of storage in the underground laboratory.

The films treated in the Tono mine were then transported to Italy by boat, thus minimizing the integrated number of low energy cosmic ray tracks compared to aircraft transportation, especially for the electron components. The transportation took about one month, the container being kept at about $15^{\circ} \mathrm{C}$ to avoid aging with high temperature. During the travel, the films were placed vertically to minimize the integrated cosmic ray flux. Nevertheless, a cosmic ray density of about 1000 tracks $/ \mathrm{cm}^{2}$ was accumulated. To suppress this background, a dedicated packing and analysis procedure was set-up. The films were vacuum packed at the Tono Refresh Facility without any spacers in between. Each pack is used for one brick so that, once the films are interleaved with the lead plates, the tracks accumulated during transportation are seen with a different alignment, and therefore can be eliminated at the analysis level. Experimental tests have validated this procedure of "virtual erasing". The initial background at the Tono mine was $43 \pm 4$ tracks $/ \mathrm{cm}^{2}$; after transportation it was $113 \pm 20 / \mathrm{cm}^{2}$ with virtual erasing and $1000 \pm 50 / \mathrm{cm}^{2}$ without.

The films were stored underground at LNGS, shielded from the environmental background by

\footnotetext{
${ }^{2} \mathrm{Keeping}$ the films at $35^{\circ} \mathrm{C}$ for 2 months is equivalent to a storage at $20^{\circ} \mathrm{C}$ for 5 years.
} 

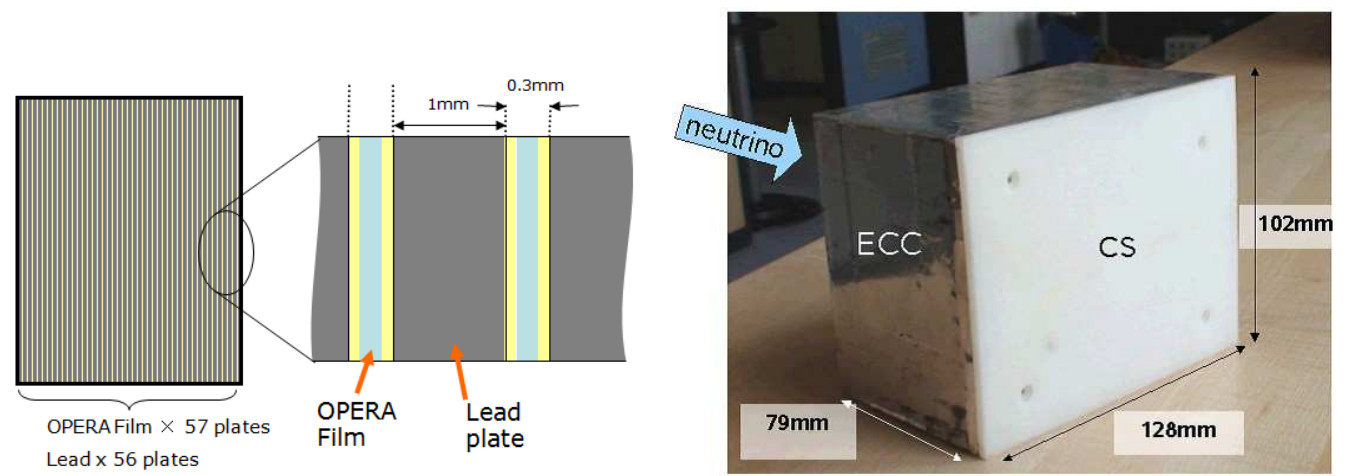

Figure 9. Left: schematic view of a brick in the target. Middle: blow-up insert showing two emulsion films and one lead plate. Right: photograph of a real brick as produced and inserted in the OPERA walls; CS is the box containing the two Changeable Sheets.
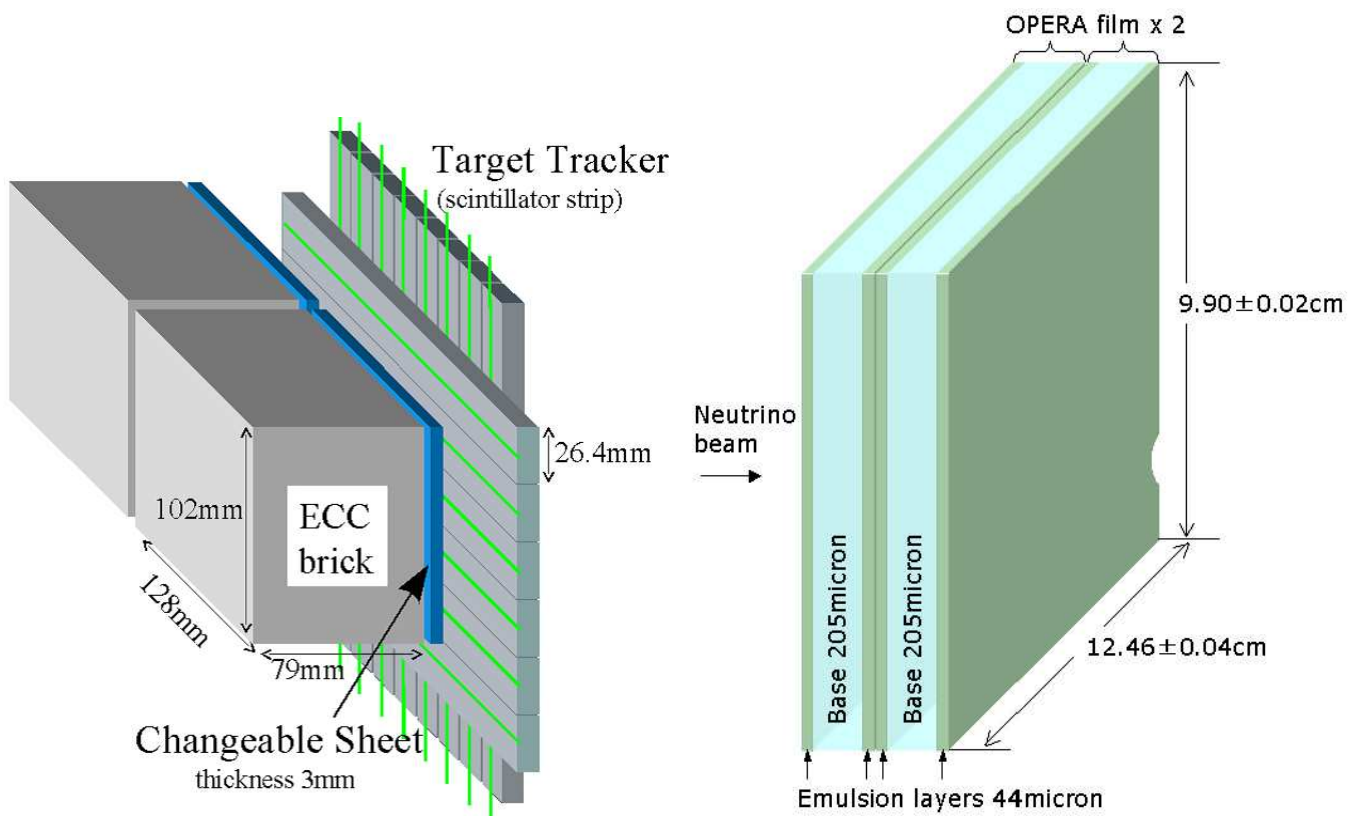

Figure 10. Schematic view of the brick with the Changeable Sheets in the target (left) and of the Changeable Sheet doublet (right).

a $5 \mathrm{~cm}$ thick iron cover and kept at a temperature ranging from 15 to $18^{\circ} \mathrm{C}$. The cosmic ray flux underground corresponds to about $1 \mathrm{track} / \mathrm{m}^{2} / \mathrm{h}$.

The film production was accomplished at Fuji Film from April 2003 to May 2005. The total number of films shipped to Gran Sasso was 9.137 million $\left(1.14 \times 10^{5} \mathrm{~m}^{2}\right)$, sufficient to build 150,000 ECC bricks.

\subsection{Bricks and Changeable Sheets}

OPERA aims to perform a quasi-online analysis of the neutrino interactions. Therefore, the target has been segmented into bricks, which can be selectively extracted from the target, developed and analyzed soon after the interaction has occurred. A brick must be large enough to contain the 
primary and decay vertex and provide particle identification and kinematical reconstruction of the event. As already mentioned, a brick is made of 57 emulsion films interleaved with 56 lead plates, each of $1 \mathrm{~mm}$ thickness. The transverse size of the brick is $128 \times 102 \mathrm{~mm}^{2}$, while the longitudinal size is $79 \mathrm{~mm}$ for a corresponding mass of $8.3 \mathrm{~kg}$ and $10 X_{0}$ as shown in figure 9. The hadronic interaction length of the emulsion gel is about $33 \mathrm{~cm}$.

For event analysis, a very precise film-to-film alignment is needed, which requires the integration of cosmic ray tracks soon after the extraction of the brick and before its development. Given the accuracy of the target tracker, the search of event tracks in the brick would be quite difficult, due to the poor signal to noise ratio resulting from the integration of cosmic-rays. This consideration motivated the use of interface emulsion films called Changeable Sheets (CS) [33].

A CS is an emulsion tracking detector acting as an interface between the electronic detectors and the brick. The CS concept was successfully applied to all past hybrid experiments like E531 [34], CHORUS and DONUT. The CS consists of two emulsion films (see figure 10) called "CS doublet". The doublet is assembled in a dedicated facility in the LNGS underground laboratory. The films are the same as the ones used for ECC bricks, but an additional refreshing process is applied in the facility to further reduce the background. The refreshing conditions are the same as in the Tono refresh facility ( $\sim 98 \%$ R.H. and $27^{\circ} \mathrm{C}$ for 3 days). The two films are packed in a lighttight envelope made of aluminum-laminated paper, which is then put into a plastic box attached downstream of the ECC brick. The background of cosmic tracks on a film is reduced to $\sim 100$ tracks $/ \mathrm{cm}^{2} /$ film. Requiring tracks aligned in the two emulsion films (4 emulsion layers) further reduces this background to about $10^{-4}$ tracks $/ \mathrm{cm}^{2}$, which motivated the choice of two films rather than only one.

Such a low background is obtained thanks to the precise alignment between the two films. This is achieved by printing five X-ray circular spots with a radius of $150 \mu \mathrm{m}$ on the CS-doublet just after brick extraction, i.e. when the CS doublet is still attached to the brick. The X-rays penetrate the two CS films and the most downstream film of the brick. An alignment accuracy between the two films of $\sim 10 \mu \mathrm{m}$ can be achieved, the precision being dominated by the lateral shrinkage of the films. An even more precise alignment $(1 \mu \mathrm{m})$ is obtained by low energy electrons emitted by radio-isotopes, as reported in [35].

\subsection{Lead plates and mass production}

The lead is used as passive target material of the ECC, because of its high density and small radiation length. The total thickness along the beam direction of one brick is $10 \mathrm{X}_{0}$, which is longer than the ECC bricks in the DONUT experiment $\left(3 \mathrm{X}_{0}\right)$ [11]. This improves the electron and $\pi^{0}$ identification efficiency [36] and the momentum resolution obtained by the multiple Coulomb scattering measurement $[13,14]$.

The type of lead had to be carefully chosen in view of the needs of long-term (5-10 years) compatibility with the emulsions. It was necessary to minimize the lead radioactivity and avoid unwanted chemical reactions [37].

Lead radioactivity is mainly due to ${ }^{210} \mathrm{~Pb}$, which decays to ${ }^{206} \mathrm{~Pb}$ emitting $\alpha(5.3 \mathrm{MeV})$ and $\beta(1.2 \mathrm{MeV})$ particles. Both create background tracks in the emulsion films and may affect the neutrino event reconstruction efficiency and purity. After dedicated tests, a maximum threshold was set on the tolerable lead radioactivity equivalent to the emission of $20 \alpha$ particles $/ \mathrm{cm}^{2} /$ day and 


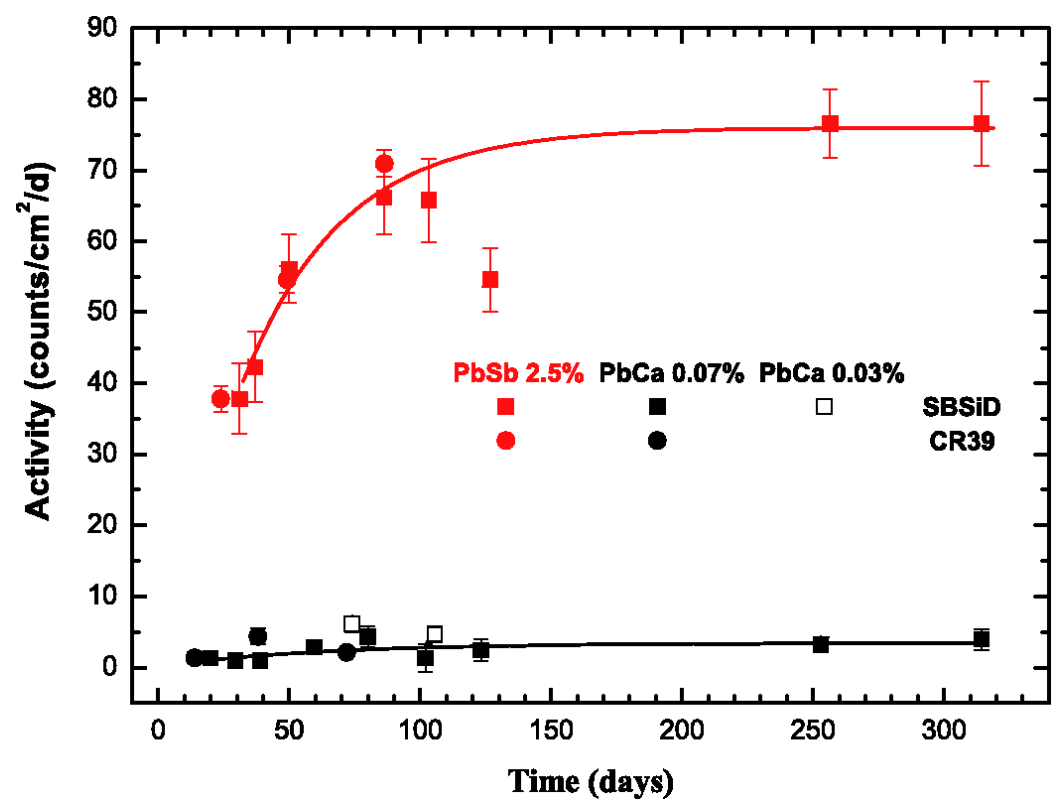

Figure 11. $\alpha$-activities of $\mathrm{PbSb} 2.5 \%, \mathrm{PbCa} 0.07 \%$ and $\mathrm{PbCa} 0.03 \%$ measured by using Surface Barrier Silicon Detectors (SBSiD) and CR39 nuclear track detectors versus the time elapsed since the production of the lead alloy plates. See ref. [37] for a detailed discussion.

$100 \beta$ particles $/ \mathrm{cm}^{2} /$ day. The Britannia mine in Australia was able to provide a large quantity of such low radioactivity lead. Although pure lead is chemically compatible with emulsions, it is too soft to be laminated and cut precisely ( $10 \mu \mathrm{m}$ planarity and $100 \mu \mathrm{m}$ on the edge) by machines. It was therefore necessary to add to the pure lead a small fraction of either Calcium (0.03 to $0.07 \%)$ or Antimony (2.5\%). The chemical compatibility between emulsions and the $\mathrm{PbCa}$ or $\mathrm{PbSb}$ alloys was tested in several steps, to evaluate the degradation of the films packed with lead plates as a function of time. Long term effects were estimated by raising the temperature of the lead and emulsion samples up to $40^{\circ} \mathrm{C}$ to accelerate the chemical processes, simulating 5 years storage at the LNGS OPERA site. It was shown that with vacuum packing $\mathrm{PbCa}$ is poisoning the emulsions while $\mathrm{PbSb}$ is safe. But when packing is done in air, both types of lead alloys are safe because the concentration of the poisoning gas is significantly reduced. Poisoning effects show up as an increase of the number of random silver grains (fog grains) in the developed emulsion films. The air packing solution was thus the safest for the bricks from the point of view of chemical compatibility.

The $\alpha$ activity of $\mathrm{PbSb}$ and $\mathrm{PbCa}$ lead plates provided by the JL Goslar company ${ }^{3}$ was thoroughly investigated [37]. It was observed that surface alpha activity in PbSb plates is much larger than in $\mathrm{PbCa}$ plates, and that it increases with time, as shown in figure 11. For $\mathrm{PbCa}$ alloys no increase with time of the surface radioactivity was observed.

The observed alpha tracks in emulsions were about $25 \mu \mathrm{m}$ long and $2.5 \mu \mathrm{m}$ thick. Since they all had about the same length, it was reasonable to assume they were emitted at the lead surface. Their range in emulsion corresponds to an energy of $5.3 \mathrm{MeV}$, compatible with the hypothesis of migration to the surface of the ${ }^{210} \mathrm{Po}$ and of its subsequent decay $\left({ }^{210} \mathrm{~Pb} \rightarrow{ }^{210} \mathrm{Po} \rightarrow{ }^{206} \mathrm{~Pb}\right)$.

\footnotetext{
${ }^{3}$ JL Goslar GmbH, Im Schleeke 108, D-38640, Goslar.
} 
The beta radioactivity was measured by silicon detectors to be within the specification, too. This justified the choice of $\mathrm{PbCa}(0.03 \%)$ for the mass lead production. Lead production at JL Goslar lasted from November 2006 until June 2008. A total of 8.4 million lead plates were produced.

\subsection{Brick Assembly Machine}

A dedicated Brick Assembly Machine (BAM) has been designed for the assembly of the bricks starting from lead plates and emulsion films. Given the very large number of bricks to be assembled in about one year and the need of shielding the emulsions from cosmic-ray radiation, it was decided to design a fully automated machine located underground near the OPERA detector site and synchronize the production and the insertion of the bricks in the detector. The most appropriate area to host the BAM was identified in an existing by-pass gallery of about $4.5 \times 40 \mathrm{~m}^{2}$ between the LNGS Hall A and B. All needed infrastructures (electrical power lines, compressed air, temperature and humidity control, network and phone lines) were installed in this area during the BAM design phase.

The Tecno-Cut $\mathrm{Srl}^{4}$ company was chosen for the BAM design, construction and assembly. Emulsion films are interleaved with lead plates and piled up by a robot. The pile is kept under pressure ( 3 bar) and it is closed in an aluminum frame. After that, the pressure is released. This procedure results in a stable and precise brick assembly with linear tolerances of about $0.1 \mathrm{~mm}$. The brick is then wrapped with an adhesive low degassing aluminum tape $(0.13 \mathrm{~mm}$ thick) to ensure light tightness. This operation also increases the mechanical stability and precision of the external surfaces of the brick. Several technical problems had to be solved for the manipulation of the brick components as well as of the assembled bricks. We solved them by using anthropomorphic robots that proved adequate flexibility and reproducibility for the positioning of the brick components. The BAM is equipped with 11 of such robots from $\mathrm{ABB} .^{5}$

The by-pass gallery hosting the BAM is divided into three independent rooms: entrance/service room, clean/white light room, clean/dark room. Similarly, the BAM machine is subdivided into 11 sub-units (stations). A lead dispenser station serving 5 piling stations is followed by a station performing the wrapping of the bricks. The other stations are devoted to the Changeable Sheets, the data matrix printing, the positioning and gluing of skates to ease brick insertion in the walls, and the automatic filling of the drum (a container for brick transportation).

The dispenser station dispatches the lead to the assembly areas in a completely automated way, so that the operators never handle directly the lead plates. The station is placed in an in-light room and manipulates customized iron boxes containing the lead. Each box hosts 18 plastic cartridges, each of them containing the 56 lead plates needed to assemble a brick. The cartridges are extracted from the iron boxes and circulate on iron shuttles at a rate of about 60 shuttles/hour. The boxes were transported from JL Goslar by trucks (120 boxes per truck) and stored in a buffer area in Hall $\mathrm{B}$ with a shipment rate of about 1.5 trucks/week. The five piling stations placed in the dark room build the lead-emulsion pile and close it at a pressure of 3 bar. Each station is equipped with two robots (lead and emulsion sides) and one hydraulic press unit. The cartridges with the lead plates

\footnotetext{
${ }^{4}$ Tecno-Cut Srl, http://www.tecno-cut.it, via L. Ariosto 116, 20099, Sesto San Giovanni (Milano), Italy.

${ }^{5}$ ABB Sadelmi S.p.A., P.le Lodi 3, Milan, Italy
} 
from the dispenser arrive to the station automatically while the emulsions are manually charged by operators. During the piling phase, three digital cameras take pictures of the front and back side corners of the brick. The images are processed on-line to detect piling errors (wrong positioning or double consecutive layers). The mean production rate of a single piling station is about 10 bricks/hour, corresponding to an overall rate of 50 bricks/hour. figure 12 shows a photo of one of the five piling and pressing stations.

The wrapping station covers all the external surfaces of the brick with a low degassing adhesive aluminum tape. It is placed in the dark room and it is equipped with one robot, two folding units, two tape dispensers and one labeling unit. Once the brick is lightproof, it is sent out to the in-light room through an automated convey. The label contains a temporary bar-code that identifies the brick and its components during its life within the machine. The wrapping cycle lasts about 50 seconds, corresponding to a wrapping speed of 72 bricks/hour.

The CS station sticks the small box hosting the CS doublet (see section 3.1) onto the brick. It is placed in the in-light room and it is equipped with one glue dispenser and one pick-and-place unit. The station is also equipped with 3 analog stroke-meters measuring the 3D size of the brick. In this way, a geometry acceptance test is performed on each produced brick.

The datamatrix printing station prints 4 codes in a $10 \times 10$ matrix format on the sides of the brick. It is equipped with a laser unit and a datamatrix code reader. Once a code is printed, it is immediately read out to verify the quality and correctness of the printing. This is the final code that uniquely identifies the brick (and its components) within OPERA.

The skates station sticks two polyethylene strips (called "skates" as they improve the brick sliding in the walls) on the bottom side of the brick. It is placed at the entrance room and is equipped with one pick-and-place unit and one capsize unit.

The drum filling station puts bricks inside a metal container called drum used to transport the bricks from the BAM to the OPERA detector site. It is located in light at the end of the BAM line. It is equipped with one rotating table (acting on the drum) and one lift to position the brick at different heights corresponding to the rows of the drum.

The BAM was delivered at LNGS in July 2006 and made operational in September 2006. The commissioning was completed in February 2007. The production lasted from March 2007 to June 2008 with an average rate of 650 assembled bricks/day. In this period, the brick production at the BAM was based on two shifts/day of 8 hours each, 5 working days a week. Each shift involved 7 operators plus 1 site manager. In January 2009 the brick production was resumed in single shift/day mode, after the delivery of the remaining lead, delayed by an accident occurred at JL Goslar in June 2008. 3415 additional bricks were assembled, for a total amount of 150036 bricks (see figure 13).

\section{The instrumented target}

\subsection{Wall mechanical structure}

As mentioned above, the OPERA bricks are positioned in walls of containers interleaved with the plastic scintillator strip planes. The large weight of the target, the required precision in the positioning of the bricks and the need of easy access to the bricks containing a neutrino interaction posed severe constraints to the mechanical structure of the walls. Each wall is an ultra-light 


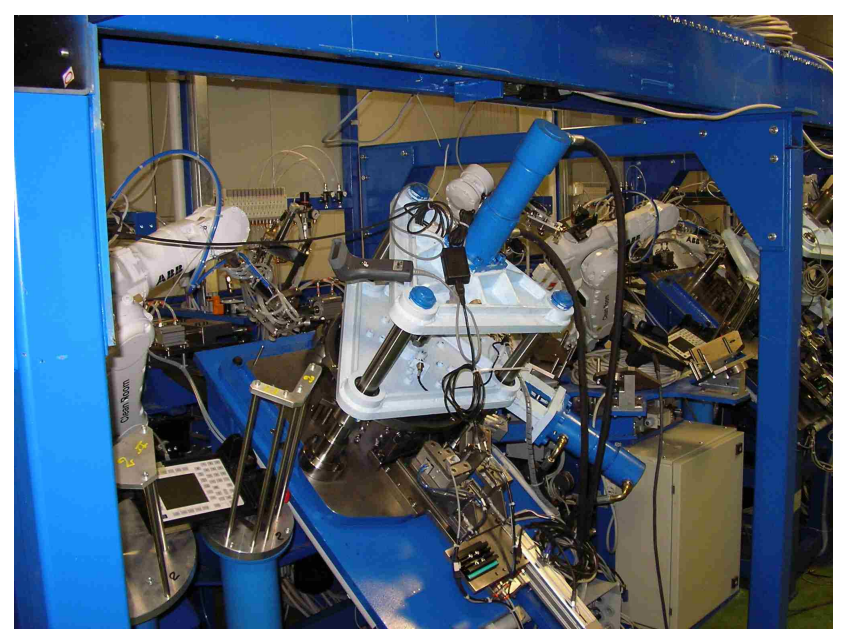

Figure 12. One of the five piling and pressing stations of the BAM in the by-pass gallery between hall $\mathrm{A}$ and B of the LNGS underground laboratory.

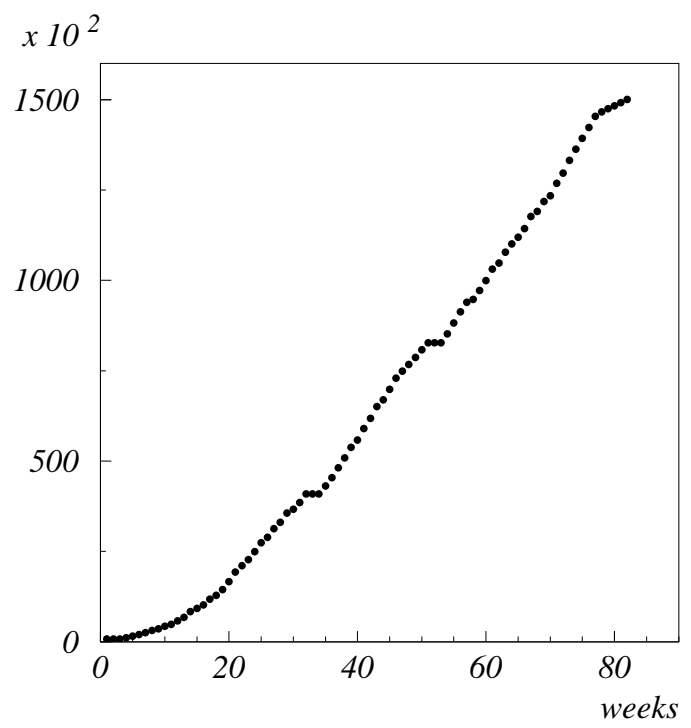

Figure 13. Number of bricks produced as a function of time (weeks). The total number of bricks produced is 150036 .

stainless-steel structure made of two identical and independent parts called "Semi-Wall" (SW), shown in figure 14. They have been designed to reach a $1 \mathrm{~mm}$ precision in the brick location and minimize the amount of passive material around the bricks. Each SW is made of 27 stainless-steel ribbons (cross section area: $0.8 \times 10 \mathrm{~mm}^{2}$, length: $6780 \mathrm{~mm}$ ) suspended from above and tensioned from below by means of a spring-tensioning system. 64 horizontal trays are welded to the ribbons (3456 welded points/SW). The ribbons sustain a strength up to $1500 \mathrm{MPa}$ while keeping the overall structure extremely light (200 kg per SW). The horizontal trays are made of U-shaped boards of stainless steel $(0.7 \mathrm{~mm}$ thickness). The overall dimensions of a SW are $3360 \times 7330 \times 85.6 \mathrm{~mm}$. One SW may house 1664 bricks (i.e. 14 tons). The extra-target material amounts to $0.4 \%$ of the total weight of the bricks. 
To reach the desired precision in brick positioning, the mechanical tolerances for the walls are very tight; the most critical are the tray pitch $(105.3 \pm 0.1 \mathrm{~mm})$, the internal width $(82.6 \pm 0.2 \mathrm{~mm})$ and length $(3358.2 \pm 0.25 \mathrm{~mm})$ of the trays, and the overall height of the wall $(7330 \pm 0.8 \mathrm{~mm})$. The achieved parallelism and planarity among the trays are at the level of $0.2 \mathrm{~mm}$.

Due to the large number of SW to be produced (124 for the whole experiment) and to the tight mechanical tolerances, the walls were built by Fantini Sud $\mathrm{Srl}^{6}$ with a dedicated automatic machine, equipped with a laser-welding tool driven by an auto-positioning system of carriages. The trays and the ribbons were held in their nominal relative position by means of a special mechanical tool till the end of the welding process. The achieved precision is in good agreement with the design specifications (tray pitch: $105.3 \pm 0.1 \mathrm{~mm}$; tray internal width: $82.6 \pm 0.25 \mathrm{~mm}$; total height: 7330 $\pm 0.6 \mathrm{~mm}$ ). The acceptance test of the tray pitch was made on each SW using mechanical gauges, while further tests were performed on a subsample of the SW with an optical theodolite and a 3D Coordinate Measuring Machine. To transport and install the walls at LNGS, 18 special frames were built. The walls were inserted from the side of the detector (see figure 14) using a custom sliding tool. Most of the installation time was devoted to alignment using optical instruments (auto level AE-5C by NIKON), a theodolite Total Station with automatic target recognition (TDA5005 by LEICA) and a few other mechanical tools. The positioning precisions achieved at the end of the installation are $<0.3 \mathrm{~mm}$ for the vertical alignment and $<0.5 \mathrm{~mm}$ for transverse and longitudinal alignment. The overall planarity of a brick wall (i.e. of the 2 Semi-Walls) is better than $1 \mathrm{~mm}$. This has been sampled during the installation with high resolution photogrammetry.

\subsection{Brick Manipulator System}

The bricks are moved in and out of their supporting trays in the walls by means of an automated system called Brick Manipulator System (BMS). The BMS has been designed to fulfill two major goals: filling the target walls with the bricks produced by the BAM and extracting on a daily basis those bricks tagged by the electronic trackers as containing a neutrino event. As no additional bricks will be produced after the initial brick installation, the BMS has also the task of filling the holes left from the extracted bricks with bricks taken from the periphery of the walls, so that the target is progressively depleted while staying compact. Since the bricks are accessible only from one side of the Semi-Wall (see section 4.1), there are two symmetric BMS machines operating independently on each side of the detector.

Each BMS is composed of an instrumented movable platform positioned inside a cradle. The cradle is placed within a $10 \mathrm{~m}$ high structure ("portico") acting like an elevator, as sketched in figure 15. Vertical runs of the platform over 8 meters are performed with an accuracy of one tenth of a millimeter by means of precision racks fixed on the portico forged pillars. The platform (see figure 15) is composed of two orthogonal moving tables allowing a precise positioning of the brick manipulating system setup in the horizontal plane (X-Z) and a precise docking approach to the SW trays. The main components of the platform consist of a 32 slot carousel to load and unload the bricks, a drawbridge used to transfer the brick from the carousel to the SW trays, pushing jacks to insert the bricks in the walls, and a small vehicle equipped with a suction grip system to extract the bricks from the wall to the carousel. The jack and the vehicle sit on a translating support in order

\footnotetext{
${ }^{6}$ Fantini Sud Srl, Anagni, Italy.
} 


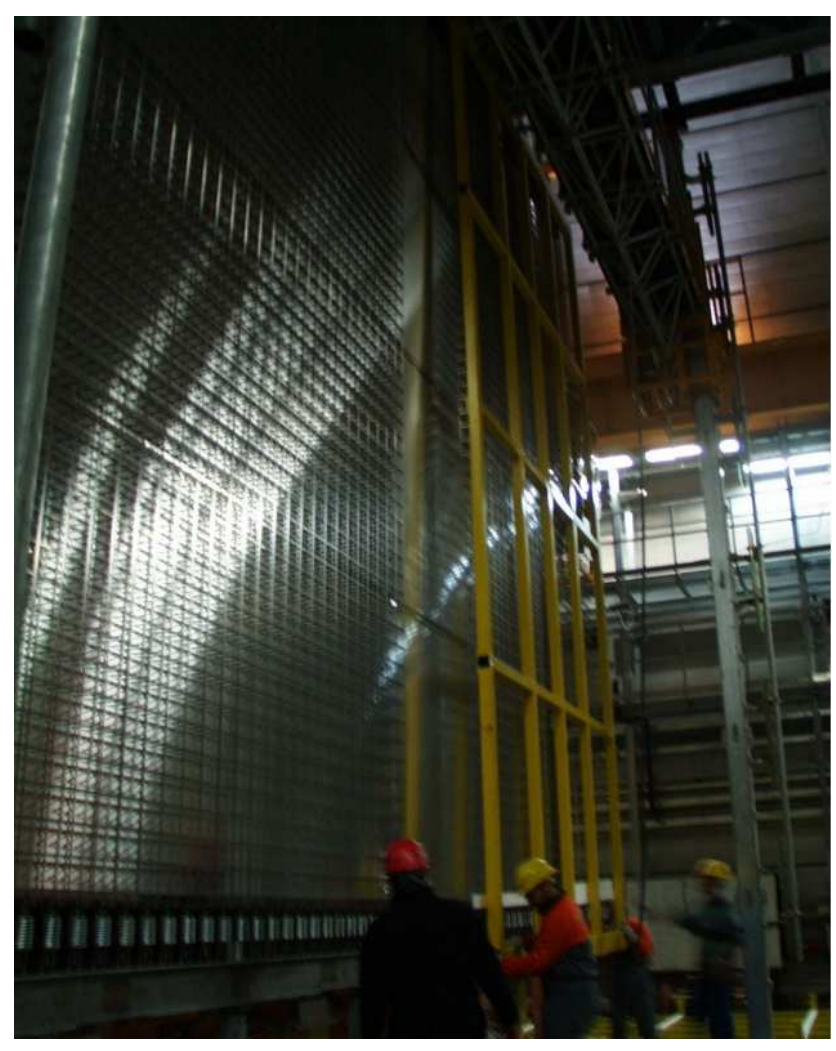

Figure 14. Installation of one OPERA detector Semi-Wall at LNGS.

for them to be aligned with the bridge depending on the brick manipulation action request. The weight of the platform is $1300 \mathrm{~kg}$ and includes four electrical cabinets containing motor control modules and power supplies for the various electrical components.

The portico is suspended to a large beam of the detector supporting structure and is able to roll along the experiment side ( $\mathrm{Z}$ axis) over $20 \mathrm{~m}$, guided by rails fixed on the floor. The portico position accuracy along $\mathrm{Z}$ is of the order of $10 \mathrm{~mm}$. The platform can move along the same axis within the portico to adjust precisely the BMS Z position with a precision of $0.1 \mathrm{~mm}$.

A BMS system involves eleven movements, each driven by a Yaskawa electric brush-less servomotor from Sigma II series. The power of the various motors ranges from $50 \mathrm{~W}$ for the small vehicle to $4.4 \mathrm{~kW}$ for the platform vertical movement. These actuators are coupled to a series of different proximity sensors to determine the absolute reference position and the end of range limits for each linear movement axis. The chosen technologies are based on inductive and photoelectric detection devices. The servomotors and sensors are controlled by a Modicon TSX Premium Programmable Logical Controller (PLC) from Schneider ${ }^{7}$ using a Profibus-DP field bus. The PLC software is designed using the PL7Pro programming platform with a mixed architecture of Grafcet and Pascal type languages. To allow the drawbridge to enter between the target tracker endcaps, a vision system has been adapted using a Siemens Simatic VS710 digital CCD camera with a $75 \mathrm{~mm}$ teleobjective, for the precise positioning of the platform in front of the SW trays.

\footnotetext{
${ }^{7}$ Schneider Electric SA 43-45, boulevard Franklin-Roosevelt 92505 Rueil-Malmaison, Cedex, France.
} 

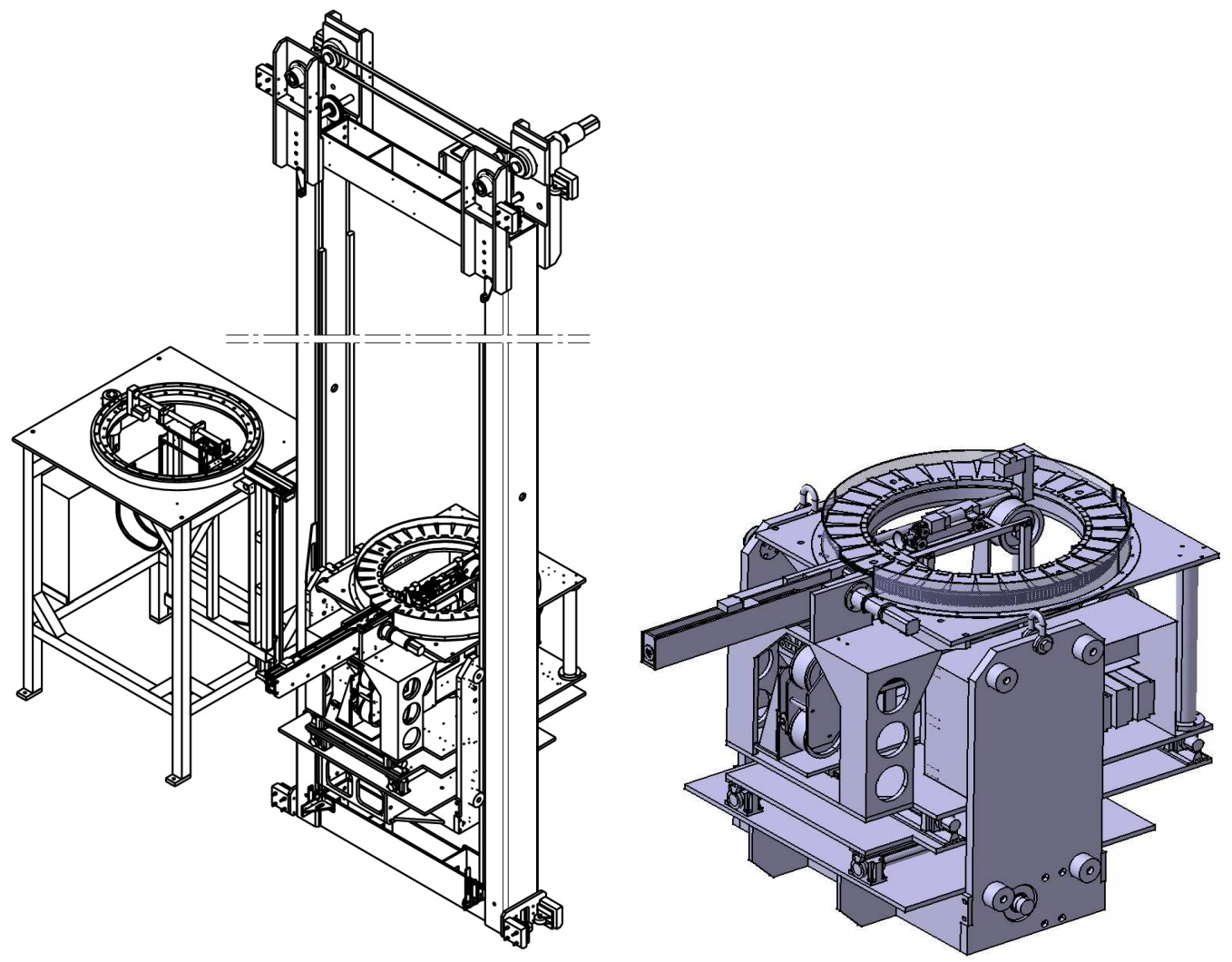

Figure 15. Left: drawing of a complete BMS system with its loading station. Right: detailed view of the platform with the various systems allowing the movements of the bricks.
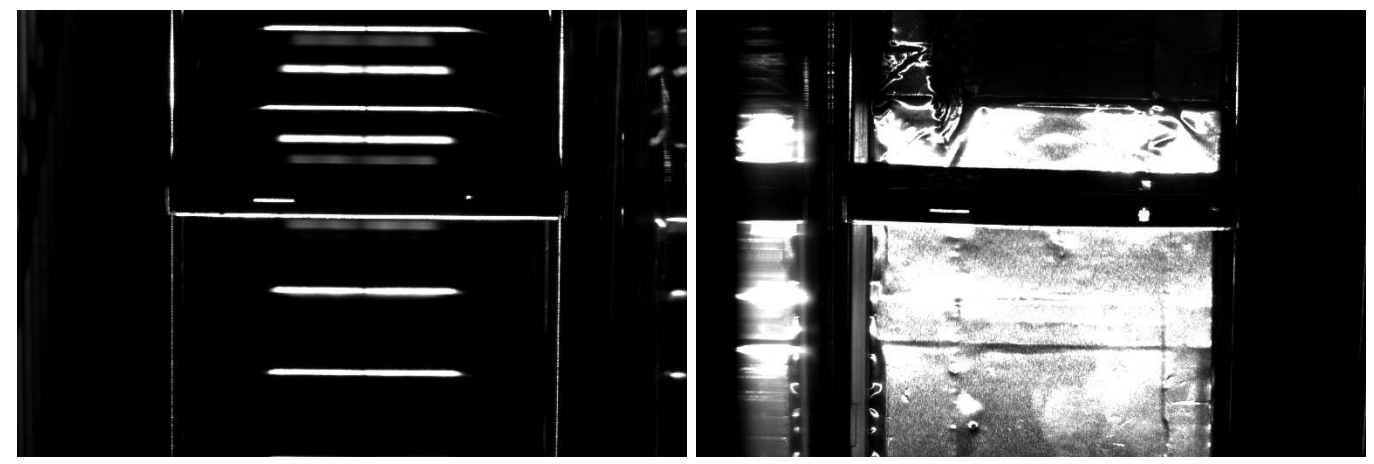

Figure 16. Pictures of a tray taken by the BMS vision system before (left) and after (right) insertion of bricks. The shadow of the tray visible on the brick surface is used to compute the distance of the brick with respect to the tray border.

This system takes the picture of the tray edge like in figure 16 and determines the position of the $10 \mathrm{~mm}$ long reference slit which is punched very precisely in each tray. The position and tuning of the camera and of its lighting have been optimised to be insensitive to the light in the experimental hall. A dedicated pattern recognition software runs in the camera processor to recognize the slit shape and to communicate to the PLC the Z,Y position, i.e. the position along the beam axis and 


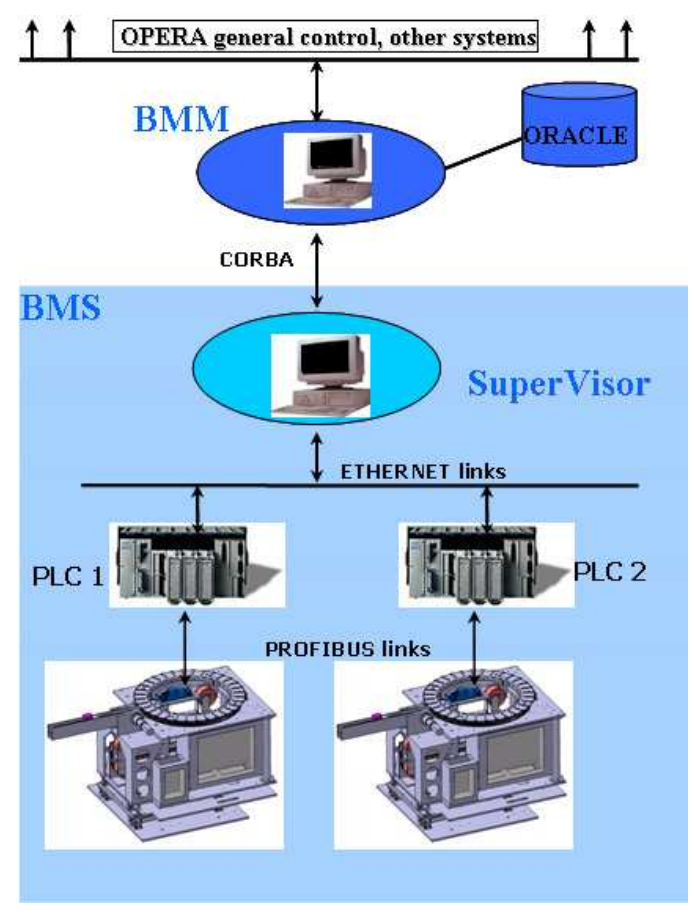

Figure 17. Schematic principle of the BMS control, including the Brick Manipulation Manager program (BMM), the supervisor program and the programs running in each PLC and issuing commands to the actuators upon signals from the sensors.

the vertical axis of the Hall $\mathrm{C}$, respectively. It is also transmitted the tray angle, the pattern finding quality and, for a tray containing bricks, the $\mathrm{X}$ position of the first brick along the tray of the SW determined with an accuracy of 1 to $2 \mathrm{~mm}$ from shadow analysis (see figure 16). The $\mathrm{Z}$ and $\mathrm{Y}$ coordinates are used to compute correction movements of the accurate BMS motors, such that the drawbridge is positioned with an accuracy of less than $0.3 \mathrm{~mm}$ to allow safe docking to the tray. The angle measurement and quality parameter are used also to accept or forbid the docking to a target tray.

In addition to the manipulators, two loading stations, one per side, have been designed and developed to allow the transfer of bricks between the BMS carousel and the transportable revolving drums. Those drums can contain 9 levels of 26 bricks corresponding to nine target trays. The loading station, sketched in figure 15, can accomplish three kinds of movements using three Yaskawa brushless motors. Depending on the drum level to access, the BMS platform height is adjusted to allow docking to the loading station with optical and inductive sensors. The brick transit system is made of a jack with a vacuum sucker, which can either push bricks on the platform carousel or pull the extracted bricks in the drum.

Several layers of software control have been developed for the BMS and for the OPERA brick manipulation management. The general scheme used for the control of the BMS is shown in figure 17. The three main components are a BMS supervisor program, a global Brick Manipulation Manager program (BMM) connected to an ORACLE database, and an operator control program to resolve BMS failures and to perform maintenance operation of the BMS. Each PLC is connected via Ethernet to the supervisor program which is responsible for the scheduling of the basic missions 


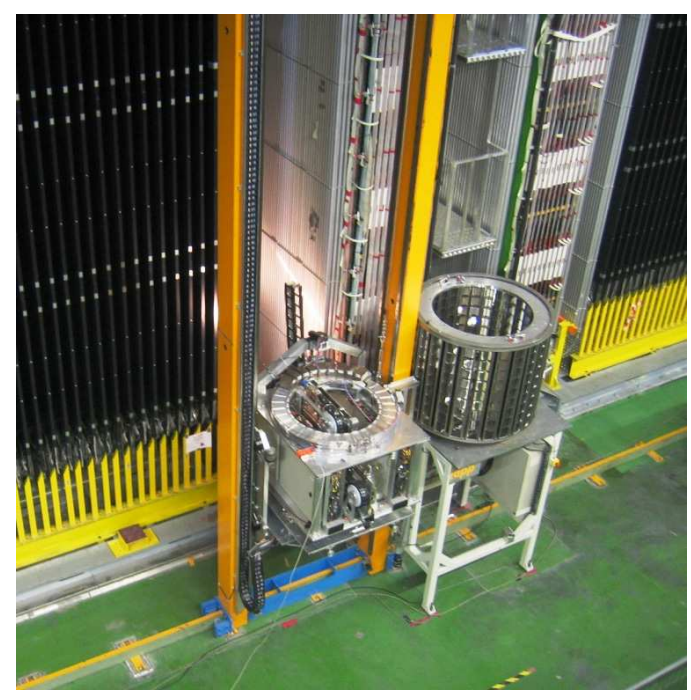

Figure 18. View of one BMS with the loading station during brick loading operation.

to be sent to the PLC according to the global high level command ordered from the BMM control panel. After receiving the return status from the missions it transmits them to the BMM database.

The bricks are uniquely identified with matrix codes, which are read by means of three Siemens HE15P Hawkeye readers installed on the BMS platform. Each brick inserted in the detector during the loading phase is managed by the BMM software component to save the location (supermodule, wall, row, cell) and to timestamp each insertion in order to keep track of all brick moves. The BMM also includes the management of the full brick life cycle from insertion to development in order to save all the actions done on the bricks. In addition, it takes care of the scheduling of the global actions and of the extraction preparation. The data persistency is based on an ORACLE database system. The BMM software organization offers a wide software component distribution and a large scalability. The communication between the components is based on the CORBA standard with an OMNIORB implementation. The supervisor and BMM programs are written in $\mathrm{C}++$ language and run respectively on a Windows and a Linux platform. Figure 18 shows a picture of a BMS attached to the loading station during the brick loading operation.

\subsection{Scintillator Target Trackers}

The total OPERA target contains 62 walls ( 31 per supermodule), each covering the $6.7 \times 6.7 \mathrm{~m}^{2}$ sensitive surface defined by the target brick wall surface. The main task of the Target Tracker detector [38] is to locate the brick in which the neutrino interactions have occurred and to provide calorimetric information on the event. The Target Tracker is made of scintillator walls suspended in between two brick walls and fixed on the beams of the main support structure. Each wall is made of a plane of four horizontal modules followed by a plane of four vertical ones, thus providing 2D track information. A module consists of 64 scintillator strips $6.86 \mathrm{~m}$ long, $10.6 \mathrm{~mm}$ thick, and $26.3 \mathrm{~mm}$ wide, read on both sides using Wavelength Shifting (WLS) fibers and multi-anode photomultipliers (PMT). A schematic view of a strip is given in figure 19. The strips have been 


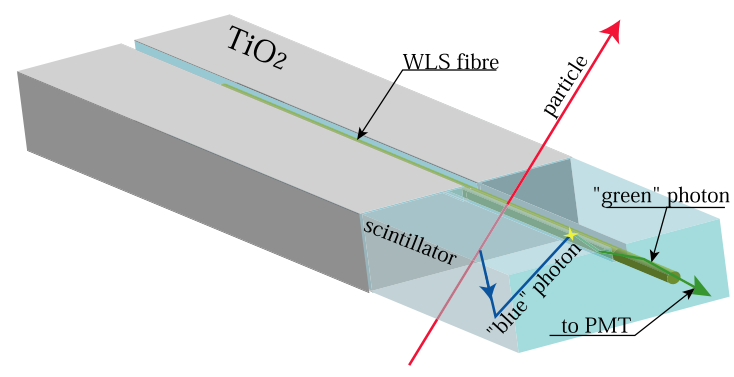

Figure 19. Schematic view of a scintillator strip with the WLS fiber.

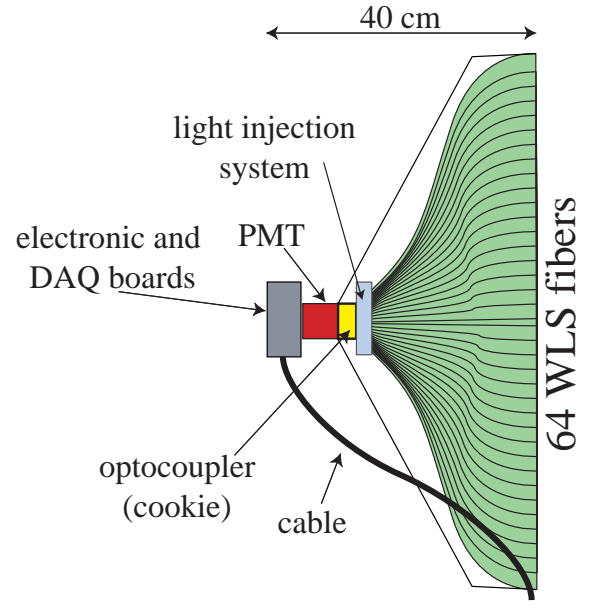

Figure 20. Schematic view of a scintillator strip end-cap with the front-end electronics and DAQ board.

produced by AMCRYS- $\mathrm{H}^{8}{ }^{8}$ after co-extrusion with a $\mathrm{TiO}_{2}$ reflective and diffusing coating for better light collection. The plastic scintillator is composed of polystyrene with $2 \% \mathrm{p}$-Terphenyl and $0.02 \%$ POPOP. A long groove at the center of the scintillating strips and running on the whole length houses the WLS fiber that is fixed using a high transparency glue. OPERA uses Y11(175) MJ non-S fibers from Kuraray ${ }^{9}$ with an attenuation length of more than $5 \mathrm{~m}$ at a wavelength of $\sim 500 \mathrm{~nm}$. The fibers are routed to the PMT photocathodes in the two module end-caps constructed by Aeriane, ${ }^{10}$ which also house the front-end electronics and acquisition cards (see figure 20). The photodetectors employed for light signal readout are based on the commercially available 64-channel Hamamatsu H7546 PMT. ${ }^{11}$ Each channel contains two sets of 12 dynodes and covers a surface of $2.3 \times 2.3$ $\mathrm{mm}^{2}$. Due to the large channel-to-channel gain variations observed in these devices (up to a factor 3 ), the gain of the preamplifiers in the front-end electronics is adjustable for each channel in order to equalize the channel response before arriving at the discriminator and charge measurement. In this way, the photo-electron detection efficiencies of all channels can be equalized at the level of $<1 \%$. To shield the PMT against the fringe magnetic field of the magnets, the end-cap covers have been built with iron STE37. This shielding is very efficient up to a magnetic field intensity of 40 Gauss.

To monitor the Target Tracker operation, a LED light injection system has been inserted near the PMT at the level of the WLS fiber opto-coupler. By pulsing this system one can easily check the status of all channels. Moreover, pulsing the LEDs on one side and measuring on the other side of the modules allows variations of the light attenuation in the fibers to be monitored. The same system, in single photo-electron mode, allows to regularly measure the gain of all PMT channels. The readout electronics is based on a 32-channel ASIC. It includes a channel by channel adjustable gain that equalizes their responses prior to measurement, a fast shaper for triggering purposes and a slow shaper to measure the collected charge.

\footnotetext{
${ }^{8}$ AMCRYS-H, 60, Lenin avenue, Kharkov 310001, Ukraine.

${ }^{9}$ Kuraray Co., Methacrylic Resin Division, 8F, Maruzen Building, 3-10, 2-Chrome, Hi-honbashi, Chuo-ku, Tokyo 103-0027, Japan.

${ }^{10}$ Aeriane S.A., rue des Poiriers, 7, B-5030 Gembloux, Belgium.

${ }^{11} \mathrm{http} / / /$ www.hamamatsu.com
} 


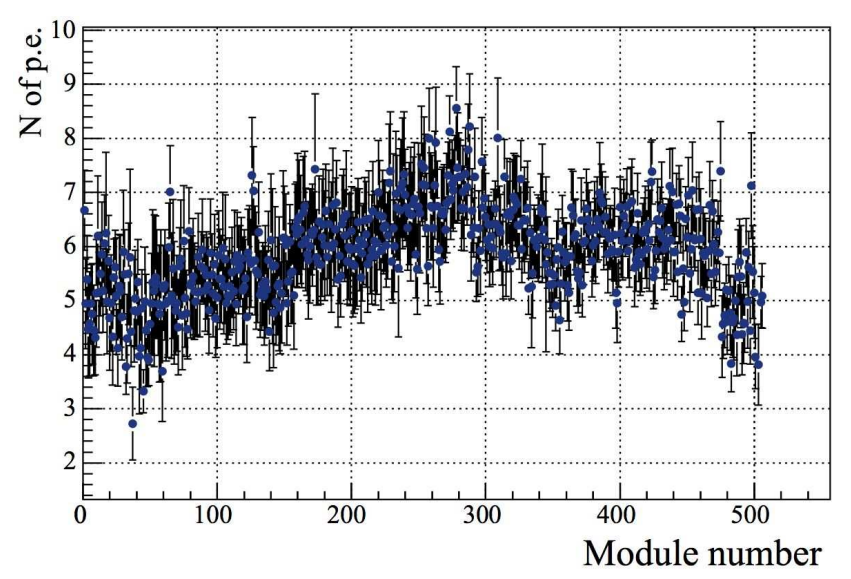

Figure 21. Average number of photo-electrons measured in the middle of the strips with $1.8 \mathrm{MeV}$ electrons from a ${ }^{90} \mathrm{Sr}$ source as a function of the module number.

In order to study the quality of the modules and to perform an energy calibration, measurements using a ${ }^{90} \mathrm{Sr}$ radioactive source, placed in a spectrometer selecting electron energies around $1.8 \mathrm{MeV}$, were taken at nine points uniformly distributed along the length of each strip. The number of photo-electrons (p.e.) is extrapolated to an energy of $2.15 \mathrm{MeV}$, which corresponds to the mean energy released by a minimum ionizing particle (m.i.p.) and the parameters are used to reconstruct the visible energy. Figure 21 shows the mean number of p.e. observed at the middle of the scintillator strips (i.e. the worst position for light collection) versus the module number (corresponding to the time of production at the company). The mean number of p.e. is 5.9. This distribution varies from 4 p.e. (lower limit of the specifications) to 8 p.e. These measurements allow to calculate the Target Tracker detection efficiency at any strip point. On the left scale of figure 22 the m.i.p detection inefficiency for a particle crossing the strip at the middle is shown versus the discriminator threshold. The mean detection efficiency for a threshold corresponding to $1 / 3$ p.e. is higher than $99 \%$. On the right scale, the individual channel trigger rate is given in $\mathrm{Hz}$. The rate measured before the brick insertion is of the order of $20 \mathrm{~Hz}$ for 1 p.e. threshold due to electronic noise, PMT dark current and environmental radioactivity.

\subsection{Veto system}

CNGS neutrinos may interact in the rock and in the concrete around the OPERA detector. Part of the secondaries from these interactions can enter the detector and may induce false triggers leading to extraction and scanning of wrong bricks. In order to reject these events, a VETO system has been installed in front of the apparatus. The VETO is made of glass resistive plate chambers (GRPC) [39, 40]. The detector is composed of two layers, each made of 8 rows composed of four units: three of them are $2.60 \mathrm{~m}$ long and $1.14 \mathrm{~m}$ high, while the fourth is $2.20 \times 1.14 \mathrm{~m}^{2}$. The global sensitive area is about $200 \mathrm{~m}^{2}$. Figure 23 shows efficiency curves for the 64 individual detectors that make up the VETO system. Each layer is equipped with horizontal and vertical copper strips with a pitch of $2.5 \mathrm{~cm}$. The vertical array consists of 416 strips, while the horizontal of 384 . In total, 1600 electronic channels collect the signals generated by the detectors. The gas system distributes the gas in parallel to each row. The flow rate is equalized by means of flow-resistors (needles), and monitored by a pressure transducer placed on the main gas line distributor. A relief bubbler 


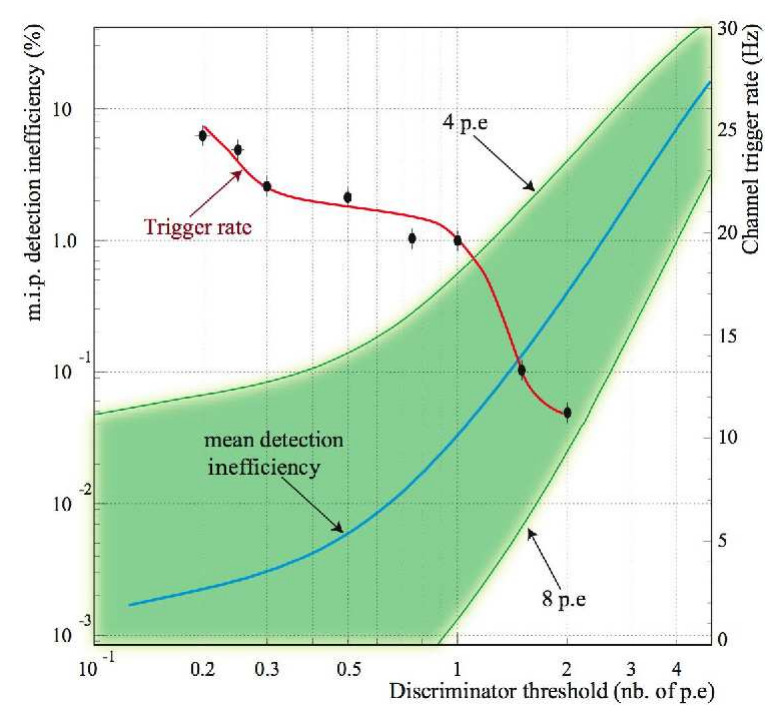

Figure 22. TT strip inefficiency (left scale) and channel trigger rate (right scale) as a function of the discriminator threshold. The blue curve corresponds to an average of 5.9 photo-electrons, and the green area is limited by the curves for 4 and 8 photo-electrons, respectively (see the text for more details). For a threshold equivalent to $1 / 3$ photo-electrons the mean strip efficiency is higher than $99 \%$, while the background trigger rate (dark current, radioactivity, etc.) stays below $20 \mathrm{~Hz}$.

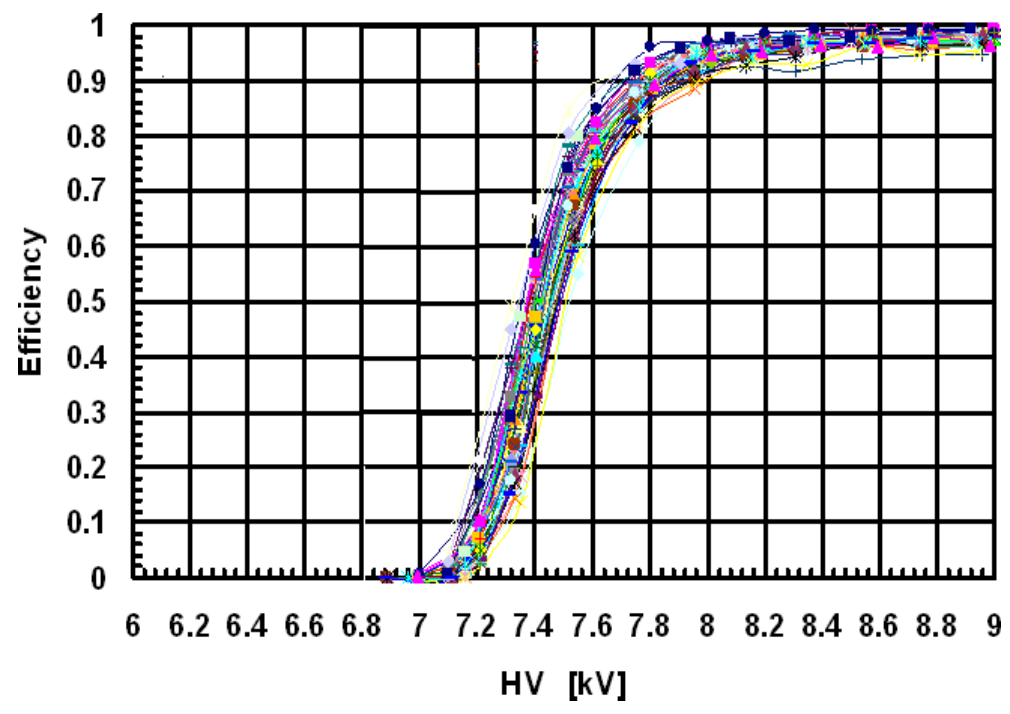

Figure 23. Efficiency of the 64 VETO chambers as a function of the applied voltage. The gas mixture is the same as for the spectrometer RPC tests (Argon/Tetrafluoroethane/Isobutane =48/48/4: see section 5.2).

is used to protect the detectors from overpressure, while an exhaust bubbler is placed downstream of the line to monitor the flow inside the chambers. Honeywell HiH-4602-C hygrometers placed at the gas input and output allow for the measurement of the water vapor contamination in the gas mixture. The high voltage distribution and the read out system are similar to those used for the spectrometer and the XPC planes, and are described in section 5.2. 


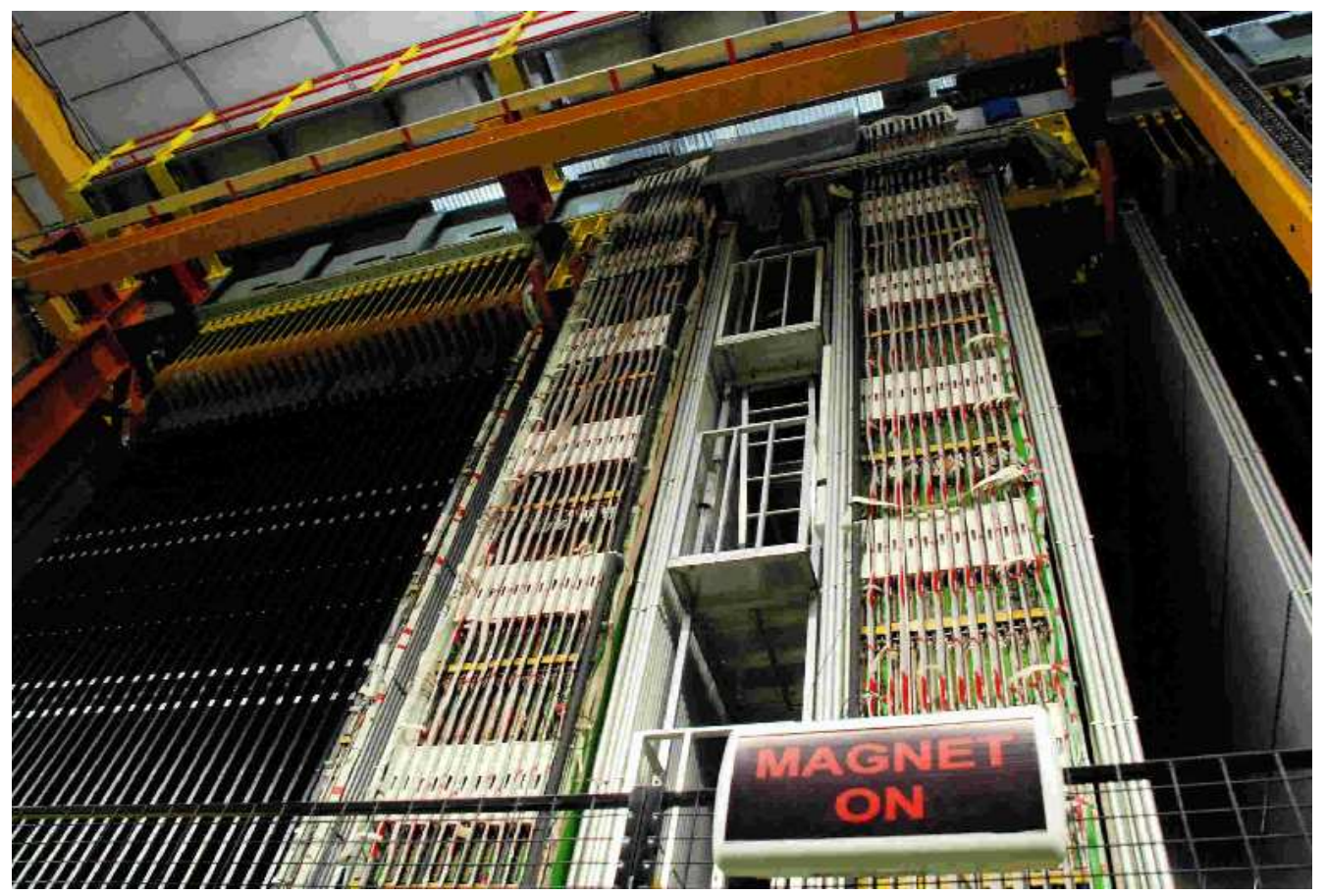

Figure 24. Photograph of one of the OPERA magnets.

\section{Magnetic spectrometers}

\subsection{Magnets}

Each of the two OPERA magnets (see figure 24) is made of two vertical walls of rectangular crosssection (arms) and of a top and bottom flux return yoke (see figure 25). The walls are built lining twelve iron layers ( $5 \mathrm{~cm}$ thickness) interleaved with $2 \mathrm{~cm}$ of air, allocated for RPC housing. Each iron layer is made of seven slabs $50 \times 1250 \times 8200 \mathrm{~mm}^{3}$. These slabs were precisely milled along the two $1250 \mathrm{~mm}$ long sides connected to the return yokes to minimize air gaps along the magnetic circuit. Slabs were bolted together to increase the compactness and the mechanical stability of the magnet that, hence, can act as a base for the emulsion target support structure.

The bottom and top return yokes consist of six steel basements (1250 mm width) and two $625 \mathrm{~mm}$ half-basements. The coil wound along the yokes is made of $100 \times 20 \mathrm{~mm}^{2}$ copper bars, whose segments are connected through bolts after polishing and gold-plating of the contact surface. Each coil is made of 20 turns in the upper return yoke connected in series to additional 20 turns in the bottom yoke. The two halves are linked by vertical bars running along the arm. Rexilon (thermohardening wooden resin) supports provide spacing and insulation of the bars. Water heat exchangers are positioned between these supports and the bars while the vertical sections of the coil are surrounded by protective plates to avoid accidental contacts.

This magnet design [41] eased substantially the construction procedures. After the positioning of the bottom return yokes and the lower coil, the installation proceeded mounting alternately the iron layers and the inner tracker planes, starting from the two innermost iron/RPC walls (see section 5.2). Once the two outermost layers were positioned, the structure was stabilized by the 
installation of the upper return yoke. The overall weight of the magnet is 990 tons and the magnetomotive force to produce the magnetic field is provided by a DC power supply located on the top platform and connected to the driving coil by means of short flexible cables. The installation of the magnets was completed in Spring 2005, while the infrastructure (water cooling system, AC power distribution, power supplies) was commissioned at the beginning of 2006. The magnets were switched on for the first time in March 2006. Results of the commissioning and details on their characterization can be found in [42, 43]. In particular, it has been demonstrated that for a current of $1600 \mathrm{~A}$ the minimum field at half height is $1.46 \pm 0.01 \mathrm{~T}$, with non-uniformities along the height not exceeding $3 \%$. The average measured field along the height is $\sim 1.53 \mathrm{~T}$. A slight flux deficit with respect to simulation (3-5\%) is observed, likely due to the effect of non-ideal mechanical contacts and steel machining. The stray field in air is in good agreement with expectations and no anomalies for the screening of the Target Tracker PMT's have been reported.

In normal conditions the magnets are operated at a current of $1600 \mathrm{~A}$, corresponding to a magnetomotive force of $64000 \mathrm{~A} \cdot$ turn. Measurements of the field along the height are performed ramping-up the magnets and integrating the induced voltage at a set of pickup coils. The power supplies are remotely controlled by a dedicated client operating through CANBUS while data generated by the power supplies (alarms, measured current and voltage etc.) are stored on the RPC/Magnet slow-control database (see section 5.2).

\subsection{RPC/XPC tracking system}

The main task of the OPERA RPC system is to reconstruct tracks inside the magnet, in particular those of stopping muons for which the momentum can be measured from their range, and to provide trigger and stop signals for the TDC readout electronics of the Precision Trackers (see section 5.3).

The RPC system is divided into two subdetectors: the Inner Tracker and the XPC. The Inner Tracker is the tracking system inside each magnet. It is composed of $22 \mathrm{RPC}$ planes of $70 \mathrm{~m}^{2}$ surface each placed in the $2 \mathrm{~cm}$ gaps between the 24 iron slabs of the magnet (see figure 25). The XPC are two RPC planes placed upstream of the magnet with the readout strips inclined by $\pm 42.6^{\circ}$ with respect to the horizontal.

The RPC technology used for OPERA is the same as that developed for LHC experiments [44, 45], BaBar [46] and Argo [47]. A sketch of the detector is shown in figure 26. Two electrodes, made of $2 \mathrm{~mm}$ bakelite with lineseed oil and volume resistivity $\rho>5 \times 10^{11} \Omega \mathrm{cm}$ at $T=20^{\circ} \mathrm{C}$, are kept $2 \mathrm{~mm}$ apart by means of polycarbonate spacers in a $10 \mathrm{~cm}$ lattice configuration. Given the very low rate capability required, there is no upper limit on the resistivity value. Nevertheless, the resistivity of the shipped plates hardly exceeds $10^{13} \Omega \mathrm{cm}$. The external surface of the electrodes is painted with graphite of high surface resistivity ( $\sim 100 \mathrm{k} \Omega / \mathrm{square})$ and protected with $190 \mu \mathrm{m}$ thick polyethylene terephtalate (PET) insulating film. An additional $250 \mu \mathrm{m}$ thick PET layer was applied during the installation on each side of the RPC to prevent high voltage discharges observed at the beginning of the installation. The inner surface of the electrodes is coated with a few micron thick polymerized lineseed oil layer. For a detailed description of the OPERA RPC detectors, their characterization and long term operation results we refer to [48].

Given the particular design of the magnet that makes it impossible to replace the RPC after their installation, special care was taken on the quality assessment of the chambers, established on the basis of three groups of tests performed at the LNGS external site: pneumo-mechanical tests, 


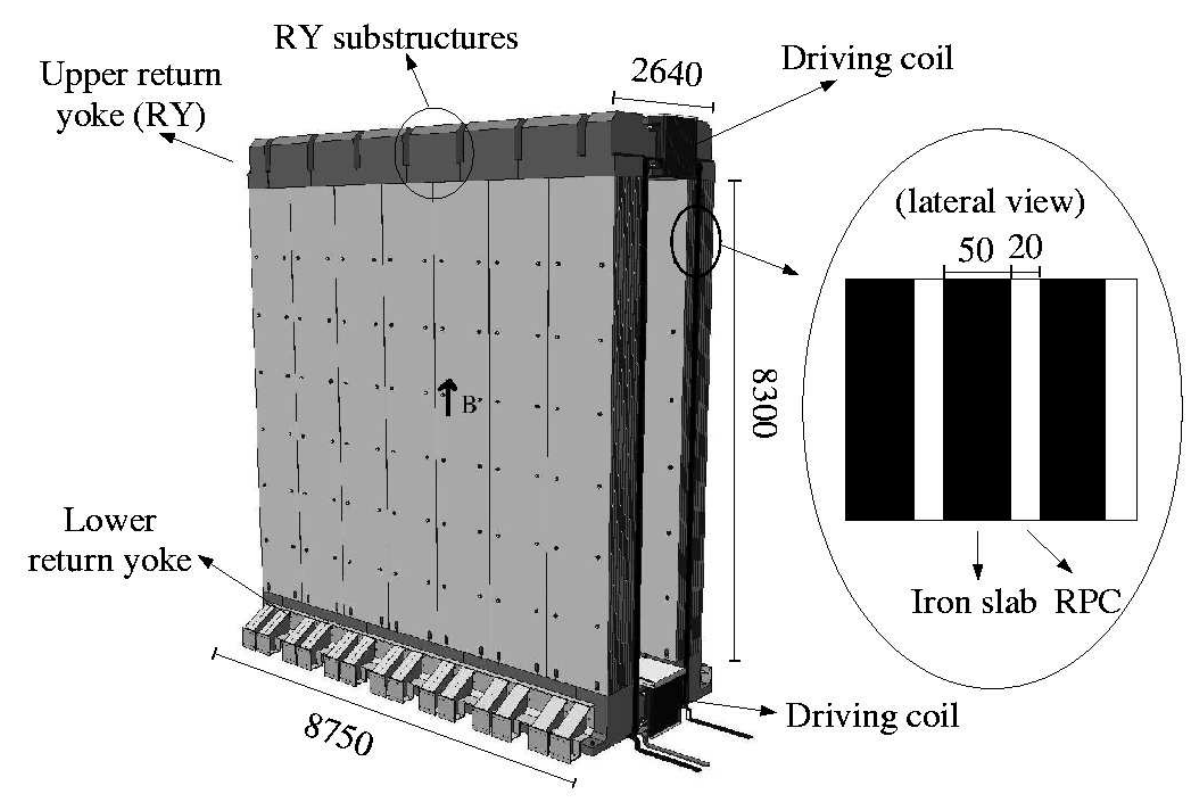

Figure 25. Three dimensional view of one OPERA magnet. Units are in $\mathrm{mm}$. The blow-up insert shows the dimensions of three of the twelve layers of an arm. The height of a slab is given as $8300 \mathrm{~mm}$.

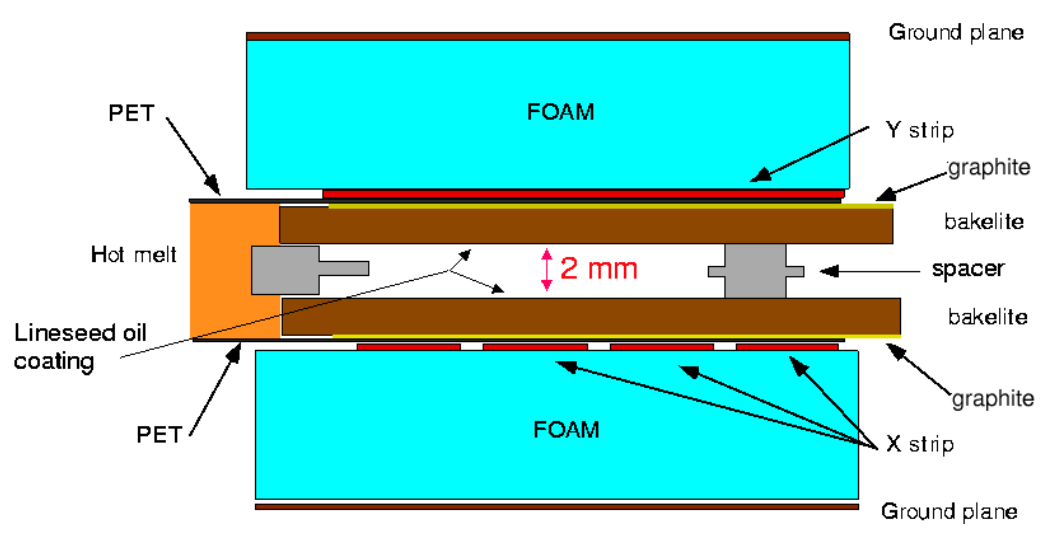

Figure 26. Cross-section of a Resistive Plate Chamber with its associated strips for the readout of the induced signal.

electrical tests and the response to cosmic rays. An outcome of these tests is presented in figure 27, that shows the efficiency curve of 32 RPC and the efficiency map of one typical chamber together with the distribution of the points in which inefficiencies are localized. The average RPC efficiency measured during the quality control test is $98 \%$ with a non-uniformity over the RPC surface smaller than $2 \%$. For a detailed description of the quality tests we refer to [49].

The Inner Tracker planes are composed of $21 \mathrm{RPC}$ of $2.91 \times 1.134 \mathrm{~m}^{2}$ surface arranged in 7 rows and 3 columns for a total of 462 RPC per magnet. In order to fit the large bolts that hold 

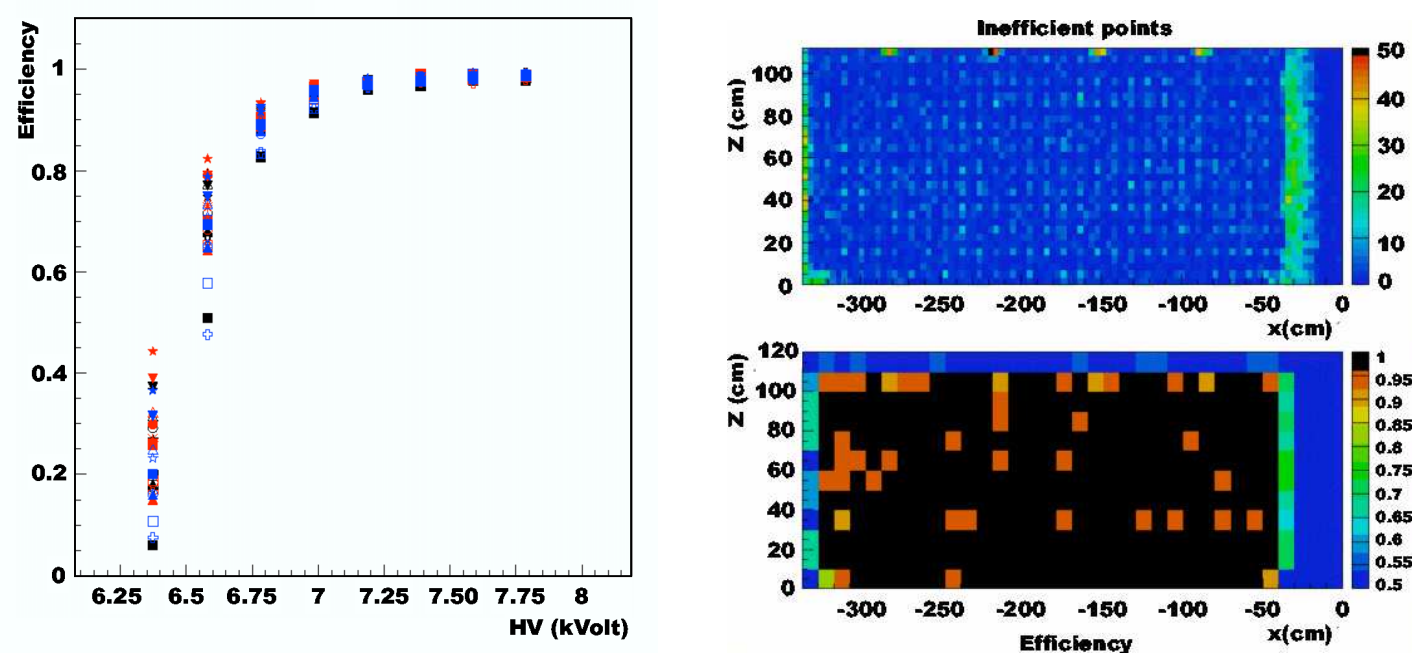

Figure 27. Left: typical RPC efficiency curves measured in the quality control tests. Right: example of the distribution of the points where the RPC is inefficient (top) and of the RPC efficiency evaluated in bins of $10 \times 10 \mathrm{~cm}^{2}$ (bottom). Note that the tests on surface have been performed with a $S F_{6}$-less mixture (Argon/Tetrafluoroethane/Isobutane $=48 / 48 / 4$ ) at an operating voltage of $7.8 \mathrm{kV}$. For normal operation the gas is different (see text) and the tubes are operated at $5.8 \mathrm{kV}$.

together the magnet (see section 5.1), 18 RPC have grooves of semi-circular shape with radius $\mathrm{R}=4.3 \mathrm{~cm}$ along one of the two long side of the RPC. Because of this particular geometry the RPC plane acceptance reaches $97 \%$. The $2 \mathrm{D}$ read-out is performed by means of $2.6 \mathrm{~cm}$ pitch and $8 \mathrm{~m}$ long vertical strips, which measure the coordinate in the bending plane, and $3.5 \mathrm{~cm}$ pitch and $8.7 \mathrm{~m}$ long horizontal strips. For each plane, the vertical strips (x coordinate) are glued on the iron wall closer to the center of the magnet. They have holes in the proximity of the magnet bolts and are shaped to run around the hole without interruptions. The horizontal strips (y coordinate) are arranged in panels of 32 strips each. The panels are supported by the magnet bolts and have grooves of semi-circular shape to match the RPC shape. The distance between two consecutive horizontal strip panels is $3 \mathrm{~cm}$. In order to get the adherence of the strip to the RPC, polyester fibers are used to fill the residual empty gap between the iron slabs.

The two XPC planes are outside of the iron magnet. They are placed $1 \mathrm{~cm}$ upstream and downstream of the first and second drift tube station, respectively (see figure 32). Both XPC planes are made of $21 \mathrm{RPC}$ detectors arranged in 7 rows but with different sizes: the first plane is $7.5 \times 8 \mathrm{~m}^{2}$ while the second is $8.7 \times 8 \mathrm{~m}^{2}$. The XPC layers are read-out by two strip panels, one on each side of the RPC, with the strip pitch of $2.6 \mathrm{~cm}$. The total number of strips per view is 416 and 448 respectively for the first and second XPC plane.

The RPC are operated in the streamer mode with a gas mixture composed of Argon, Tetrafluoroethane, Isobutane and Sulphur Hexafluoride $\left(S F_{6}\right)$ in the volume ratios 75.4/20/4/0.6 [50]. Each RPC row is flushed independently at the rate of 5 refills per day. With this gas mixture the RPC are operated at the voltage of $5.8 \mathrm{kV}$ with a current of less than $100 \mathrm{nA} / \mathrm{m}^{2}$ as shown in figure 28 . The high voltage (HV) is symmetrically split between the positive and the negative electrode to ease insulation from ground. The HV is distributed in such a way that the negative voltage is spread 

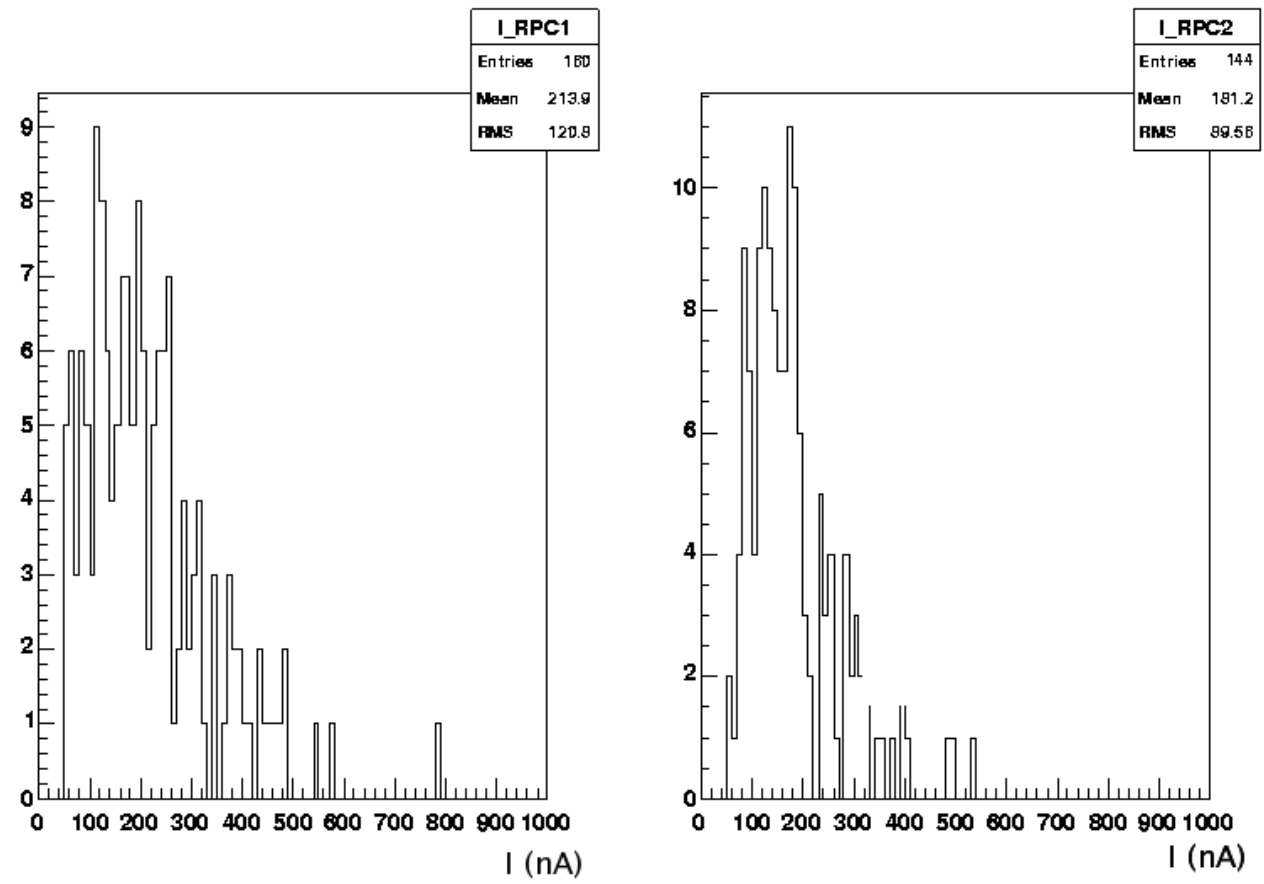

Figure 28. Distributions of the RPC currents as read by the current-meters for three chambers in series $(9.8$ $\mathrm{m}^{2}$ ). The two plots refer to the RPC of the first (left) and second (right) spectrometers, respectively.

by passive distributors, that in turn split one channel in 63 lines, i.e. 3 RPC planes. The positive HV channels are fed to a custom current-meter [51], which distributes one input to three RPC rows and measures the current flowing in each row separately with a resolution of about $1 \mathrm{nA}$. The total number of HV channels is 144. The HV system is controlled by the RPC slow control system [52], which also monitors and records the RPC currents measured by the current-meters, the temperature of the RPC measured by 10 NTC thermistors installed on each RPC plane, the status of the gas system and of the low voltage of the readout electronics. The same system is used to store the temperature of the magnet coils and the status of the magnet power supply.

The signals from the strips of the RPC and XPC are collected by means of $15 \mathrm{~m}$ long twisted pair cables and carried to the top of the magnet where the read-out electronics [53] is located. The readout is digital and makes use of LVDS receivers as comparators. Those devices, because of their differential inputs and high common mode rejection ratios allow for a good matching of the signals transmitted through twisted pair cables. The RPC Front-End Boards [54] (FEB) consist of 64 channel discriminators, each individually maskable and with input polarity and thresholds that can be set in groups of 32 channels. The FEBs are self-triggerable through a 32 channels Fast-OR which is used to form the RPC plane trigger and to start the readout chain. Each RPC plane is read-out by nine FEBs. A dedicated trigger board allows for the definition of special triggering conditions between the 11 RPC planes of each spectrometer arm and an external validation signal.

In addition to the read-out electronics, the XPC and seven RPC layers in each spectrometer are instrumented with dedicated Timing Boards [55] (TBs), following the scheme illustrated in figure 32. The timing boards are high impedance discriminators plugged on the read-out strip 


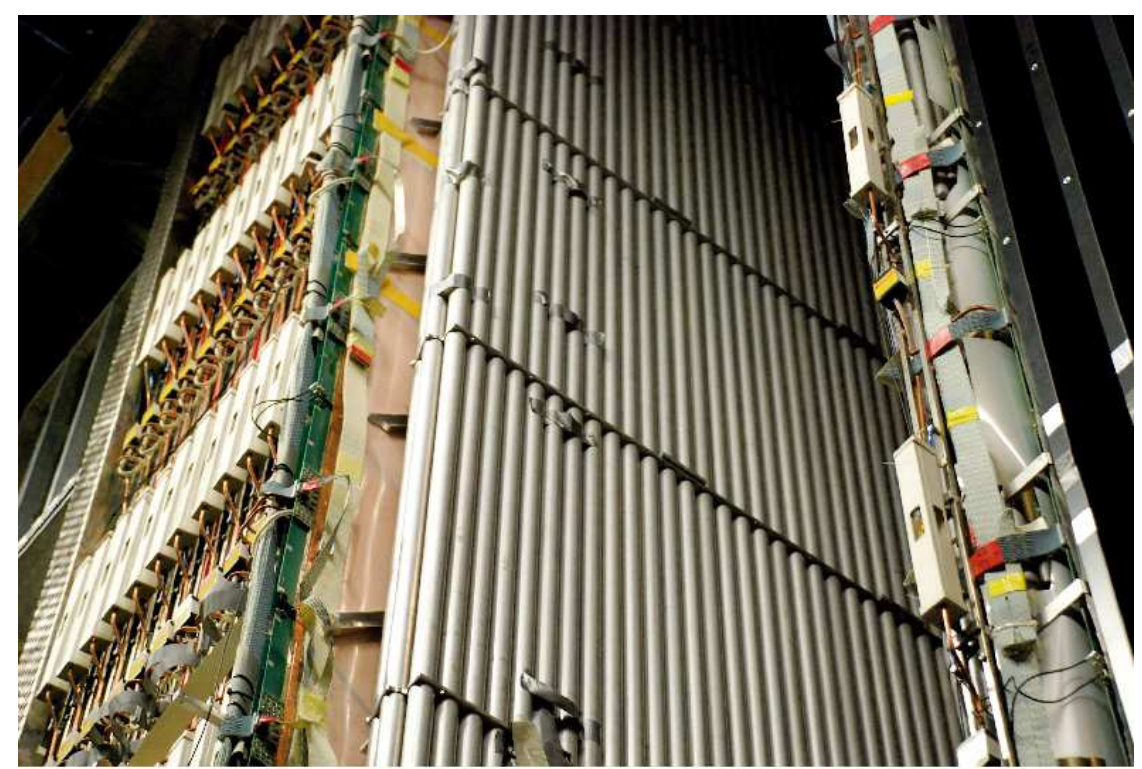

Figure 29. Picture of the high precision drift tubes (PT).

panels and operated at low threshold for timing applications. They are used to trigger the Precision Trackers (PT) and to give a common stop to their TDCs (see section 5.3).

Each TB is made of 16 positive input discriminators, whose outputs are OR-ed into a $60 \mathrm{~ns}$ wide shaped signal in positive ECL logic. One RPC plane is served by $14 \mathrm{TBs}$, connected to a custom OPE (Or Plane Electronics) board used to generate a global OR for the entire layer. The global OR signals, in positive ECL logic and 110 ns wide shaped, are fed to dedicated PT Trigger Boards. Because of the inclined strips, XPC1 and XPC2 are read out by 27 and 28 TB, respectively, for a total of 4 OPE boards. Each spectrometer is therefore served by $153 \mathrm{~TB}$ and 11 OPE boards. Both the TB and the OPE boards outputs are sent into the TDC boards that are used to read the PT, and therefore they can be used for time-of-flight measurement exploiting the excellent time resolution ( $2 \mathrm{~ns}$ ) of the RPC. Also the OPE boards are managed by the RPC slow control system, which performs the threshold setting, masks possible noisy TB and monitors the OPE boards output rate. At the operating voltage of $5.8 \mathrm{kV}$ and for a typical threshold of $15 \mathrm{mV}$, counting rates of $\sim$ $20 \mathrm{~Hz} / \mathrm{m}^{2}$ have been measured.

\subsection{Precision trackers}

The Precision Tracker (PT) [56] shown in figure 29 is used together with the other parts of the muon spectrometer for the muon identification, the determination of the sign of the muon charge and its momentum. Muons are deflected by the two arms of the magnet (see section 5.1) with opposite magnetic field direction. The PT measures the muon track coordinates in the horizontal plane as shown in figure 30 .

It is made of 10000 vertical drift tubes of $8 \mathrm{~m}$ length with an outer diameter of $38 \mathrm{~mm}$, a wall thickness of $0.85 \mathrm{~mm}$ and a sense wire diameter of $45 \mu \mathrm{m}$. The PT consists of 12 chambers (PT walls, 6 per supermodule), each covering an area of $8 \times 8 \mathrm{~m}^{2}$ and made of four staggered planes (see figure 33). In each tube, the sense wire is held at both ends without any intermediate support, a 


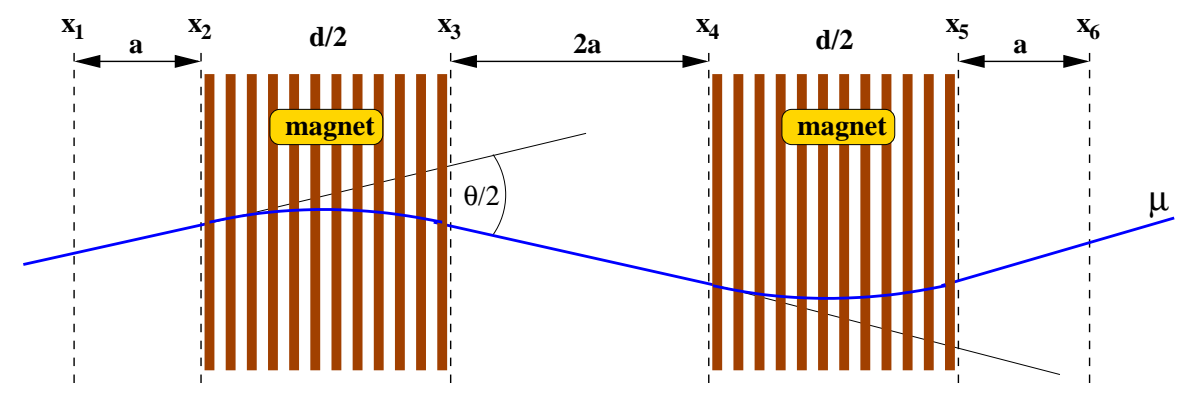

Figure 30. Schematic layout of one half of the muon spectrometer. The six drift tube chambers (PT) are denoted by $x_{1}-x_{6}$. With three chamber pairs the momentum can be extracted from two independent measurements of the deflection of the charged particle in the magnetic field.

technical challenge never realized before on such a length. The wire position for the whole module is independent of the tube position. Thus, a wire position accuracy better than $150 \mu \mathrm{m}$ can be reached. The spatial single tube resolution is measured to be less than $300 \mu \mathrm{m}$ (rms). In order to minimize the number of gas connections, the gas flow between the tubes is obtained by using milled gas channels inside both end plates. The single tube hit efficiency has been measured to be larger than $98 \%$ (see figure 24 of ref. [56]) while the "single tube track efficiency", i.e. the product of the single tube hit efficiency and the probability that the hit gives the correct drift radius, is $\sim 90 \%$ (figure 25 of ref. [56]).

The OPERA PT drift tubes are filled with a $80 \%$ Argon $20 \% \mathrm{CO}_{2}$ gas mixture and are run at an absolute pressure of $1005 \pm 5$ mbar. The total active gas volume of the PT amounts to $80 \mathrm{~m}^{3}$ and is exchanged during stable operation once in 80 hours, without recycling. Both Argon and $\mathrm{CO}_{2}$ are stored in the liquid phase outside the experimental hall. Heat exchangers and compensating reservoirs ensure a gas temperature close to $15^{\circ} \mathrm{C}$. Both gases are mixed with mass flow controllers within an accuracy better than $1 \%$ per component and leave the mixing station with an absolute pressure of $1500 \pm 5$ mbar. To distribute the mixed gas to the twelve PT walls, four subunits are located in the interspace in-between. They are connected to the mixing station via stainless steel tubes, each distributing the mixed gas to three PT walls. Each PT wall is built from 15 or 17 modules and divided into six subvolumes consisting of two or three modules each. Each subvolume is individually supplied with a manual floating type flow-controller. The exhaust line of each subvolume is split and leads via magnet-valves to either the global exhaust or to a gas quality monitoring system.

The gas density has to be uniform to minimize variations in the drift time spectrum. Thus, the drift tubes are operated at an absolute pressure. Each subunit is indeed equipped with a precise pressure transducer, a regulation valve and a magnet valve that opens at $1010.0 \pm 0.1 \mathrm{mbar}$ and closes at $1000.0 \pm 0.1 \mathrm{mbar}$ (see figure 31 ), controlling the pressure of three PT walls simultaneously. Since the Gran Sasso underground halls are a low radioactivity environment, gas quality monitoring through radioactive sources cannot be implemented. Therefore, in order to observe possible contaminations of the drift gas with air, commercial oxygen monitoring devices are used in every subunit [57]. For normal operation, an oxygen contamination of up to $100 \mathrm{ppm}$ can be tolerated.

The PT trigger [58] is fed by the RPC/XPC timing boards (section 5.2) and has been optimized 


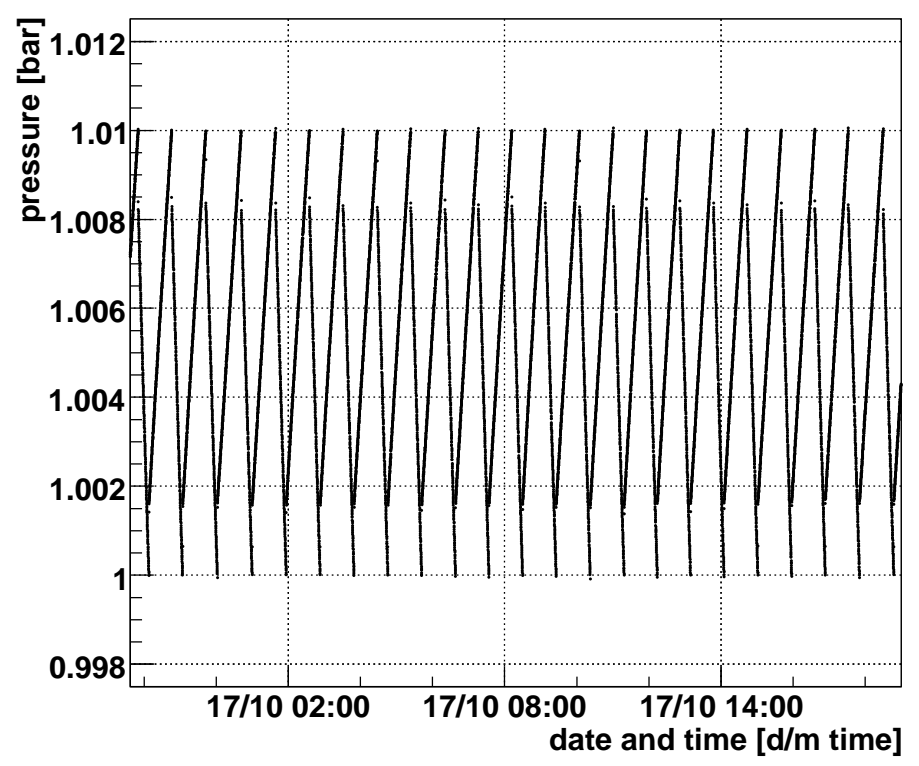

Figure 31. Regulated pressure in the PT subunit "1" over a period of several hours.

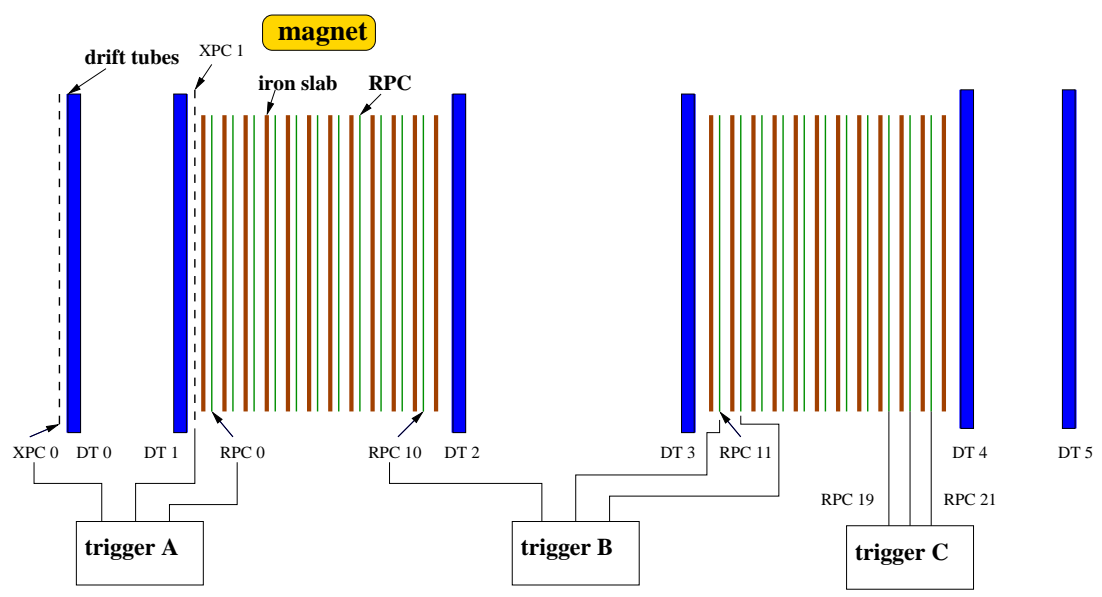

Figure 32. Overall trigger scheme for one supermodule.

for through-going tracks, stopping muons and hadronic showers. It is also sensitive to cosmics and provides up/down discrimination from the measurement of the time-of-flight. Figure 32 shows the overall trigger scheme for one spectrometer supermodule.

The trigger system is made of three identical stations $(A, B, C)$, each managing the readout of one pair of PT-walls. Each plane of RPC/XPC equipped with timing boards generates a fast OR signal sent to the corresponding trigger station. As shown in figure 32, the first PT wall pair of each supermodule (DT0+DT1) is managed by a trigger station (Trigger A) fed by two XPC and one RPC planes. The other pairs, managed by Trigger $B$ and Trigger $C$, receive signals from the closest RPC. If a majority of two out of three RPC/XPC planes occurs, the station generates the TDC stop signal and starts the readout chain for the corresponding PT data.

Propagation delays can be compensated offline using the spectrometer track reconstruction. 


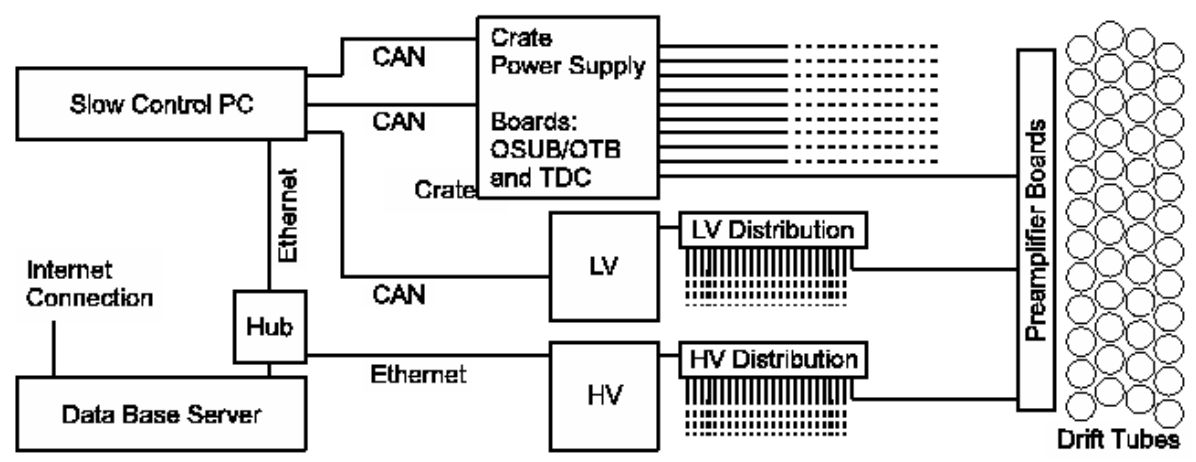

Figure 33. Scheme of PT Slow Control system.

Moreover, different cable run times can be determined by TDCs integrated in the trigger system and can be corrected for by several implemented delays. The trigger is monitored and controlled through CANBUS (Controller Area Network bus) and the majority configuration can be set by CPLD (Complex Programmable Logic Device).

To increase the efficiency for cosmics coming at steep angles, a special configuration was designed where all tubes are read out even if only one station generated a trigger. The PT trigger rate per supermodule is about $2 \mathrm{~Hz}$.

The PT slow control manages the power supplies for the high voltage, for the operating low voltage, for the crates as well as the control of the OPERA Support Boards (OSUB) and the Trigger Boards (OTB). All relevant parameters are written into a data base on a server connected to the PT slow control PC. Figure 33 shows the scheme of the PT slow control and its components. To provide the drift tube wires with high voltage a CAEN ${ }^{12}$ power supply is used for all PT and is controlled through Ethernet [59]. Two power supplies per supermodule provide the operating voltage for the analog and the digital circuits. The LV is distributed to the drift chamber boards by means of six distribution units. Both, the LV supplies and the power supplies of the crates, can be controlled via CANBUS [60]. Commercial software by WIENER ${ }^{13}$ has been employed for the readout of voltages, currents and for remote control of the crates. The OSUB and the OTB allow settings of trigger configuration and thresholds; moreover, they manage the sending of test pulses and the measurement of the temperature along the tubes [61].

The PT time measurement is done by a customized Time to Digital Converter (TDC) system developed in cooperation with MSC. ${ }^{14}$ Since the PT drift time spectrum ranges up to $1.6 \mu \mathrm{s}$, the TDC has a measuring range of $3.2 \mu$ s with a Least Significant Bit of $1.5 \mathrm{~ns}$. In addition the TDC measures the pulse width of the start signal, which is given by the discriminated signal from the drift tube. The pulse width is classified into short $(<12.50 \mathrm{~ns})$, medium $(12.50-81.25 \mathrm{~ns})$ and large ( $>81.25 \mathrm{~ns})$ pulses. This measurement can be used to discriminate noise signals from drift signals, since noise signals have a short pulse width while regular signals are of medium and large pulse width.

\footnotetext{
${ }^{12}$ CAEN SpA, Viareggio (LU), Italy.

${ }^{13} \mathrm{http}: / /$ www.wiener-d.com

${ }^{14}$ MSC Vertriebs GmbH, Industriestrasse 16, 76297 Stutensee, Germany.
} 
Each TDC board provides 96 measuring channels split into 12 TDC chips with eight measuring channels each. 105 TDC boards have been installed to readout all the 12 PT walls. Additional calibration channels located in the TDC chip compensate for slow fluctuations induced by variations in temperature and voltage.

The TDC can sustain rates of up to $100 \mathrm{kHz}$. Since the readout is performed by a $10 \mathrm{Mbit}$ Ethernet connection, this rate is limited to $4.2 \mathrm{kHz}$, which is fully compatible with the requirement of the experiment.

\section{Detector alignment}

The OPERA apparatus is made of many independent components which had to be precisely assembled and whose relative positions have to be known for track reconstruction, momentum measurement and brick finding. Given the very low flux of cosmic rays in the underground laboratory the experiment makes a heavy use of survey techniques in order to measure the relative positions of its components. The goal is to record the 3D positions of the scintillator strips, RPC strips and bricks with an accuracy of better than $1 \mathrm{~mm}$. The PT in the spectrometer should be surveyed with an accuracy better than $300 \mu \mathrm{m}$ to match the intrinsic position resolution of the chambers. These specifications should be met on an overall scale of about $10 \times 10 \times 20 \mathrm{~m}^{3}$, which is the volume of the detector and of its support structure for a total weight of about 4 kton.

In addition, specific measurements were performed to monitor the evolution of the mechanical structure during the loading of the bricks, and the installation and commissioning of the BMS. Finally, the OPERA reference system was also linked to the global geodesic system of the CNGS. The alignment operations were organized in two steps. The first operation was the characterization of the components and the follow-up of the installation to get the initial positioning and assembling of all subsystems with the best accuracy. Then, at the completion of the installation phase, a final survey was carried out. It included a precise measurement of the positions of the components to perform software corrections at the track reconstruction level and the monitoring of the positions as a function of the brick loading.

In order to fulfill the above tasks, we set up a variety of customized alignment techniques based on two measurement technologies. The first one, called close range targeted digital photogrammetry, was based on a commercial system made by $\mathrm{AICON}^{15}$ and mainly used for the first quality checks and for the characterization of the intrinsic mechanical accuracy of the components (target tracker planes, brick walls and support structure). The second one, used for both fast positioning assembly and final survey, and linking all the measurements in a global OPERA reference system, was based on an automatic 3D optical system with about $0.2 \mathrm{~mm}$ accuracy, namely, the total station TDA5005 by Leica. ${ }^{16}$ This instrument turned out to be very flexible and quite easy to use even for non-specialists. Both technologies need targets, so a large number of mechanical adapters was developed in order to install targets in the key positions to be surveyed.

The positioning accuracy of the bricks is driven by the positioning of the walls and their construction accuracy, which is at the level of $0.2 \mathrm{~mm}$ (see section 4.1). This intrinsic accuracy was checked with photogrammetry that demonstrated the achievement of an accuracy of a few tens of

\footnotetext{
${ }^{15}$ AICON 3D Systems GmbH, Biberweg 30 C, 38114 Braunschweig, Germany.

${ }^{16}$ Leica Geosystems S.p.A. Via E.L. Cerva 110, 00143, Roma, Italy
} 
microns over volumes of many hundreds of cubic meters by taking about 200 pictures and using about one hundred targets uniformly distributed over a surface of $7 \times 7 \mathrm{~m}^{2}$.

The positioning and survey of the brick walls and TT was done by taking measurements in a few tens of reference points with the TDA5005. At the end, it has been possible to parametrize each scintillator strip by knowing the 3D position of the two extreme points. Each Semi-Wall (see section 4.1) was parametrized in 3D as a flat rigid surface. These measurements can provide the position of the bricks along the vertical coordinate and the beam coordinate. The knowledge of the third coordinate, the one of the bricks along the tray length relies instead on the measurements performed at the insertion by the BMS ( $0.2 \mathrm{~mm}$ accuracy) and on the knowledge of the brick dimensions.

In order to link all measurements, a network of more than 100 reference points was installed in the Hall $\mathrm{C}$ at different heights and uniformly distributed all around the detector. During the construction of the network each reference point was measured on average 8 times from different positions. The residuals on the determination of positions of the reference points were ranging from 0.02 to $0.08 \mathrm{~mm}$. The network was designed in order to have about 20 reference points visible from any possible position and to be able to achieve a positioning accuracy of the TDA5005 in the OPERA system equal or better than $0.2 \mathrm{~mm}$.

The survey of the PT needed special care. Each PT plane was completely characterized by measuring a network of 128 reference points typically distributed on 8 vertical levels with $1 \mathrm{~m}$ pitch. Each vertical level was subdivided horizontally in 16 measurements distributed over $8 \mathrm{~m}$. The points were measured with the TDA5005 by surveying from the side of the detector and exploiting a quite narrow window along the beam axis around the plane. All these measurements were performed after having oriented the instrument in the OPERA reference system.

The evolution of the geometry of the target structure during the loading with the bricks was monitored by surveying the shape and absolute position of the top I-beams of the support structure that holds the walls and the TT. Similarly, the absolute position at different heights of the walls and of the TT planes have been recorded. The elongation of the walls was parametrized by using a simple model based on a set of springs connected in series. The model including the I-beams and the walls was able to reproduce the measurements taken at different loading configurations with an accuracy better than $1 \mathrm{~mm}$.

\section{Data acquisition and software}

Profiting from the intrinsic low data rate of the detector, the DAQ system was built with the concept of the so-called "smart sensors" in which each detecting element is readout by an independent micro-processor board which sends its data over a standard Ethernet network. In the following, the term sensor refers to a unit composed of a controller motherboard and an Ethernet controller mezzanine.

The main requirements to the DAQ system are triggerless operation mode, accurate timestamping locked to the GPS (to correlate off-line events with the CNGS), continuous running capability with low deadtime, modular and flexible hardware/software architecture (trigger schemes, on-line and off-line filters etc). 


\subsection{Global DAQ architecture}

The central elements of the DAQ system are mezzanine boards hosting a micro-processor ETRAX 100LX from AXIS; it is a 32-bit RISC CPU with Linux 2.4 OS running at $100 \mathrm{MHz}$ and presented in a Multi Chip Module (MCM) with 4 Mbytes of flash memory, 16 Mbytes of SDRAM and Ethernet transceiver [62]. The mezzanine also hosts a sequencer (ALTERA FPGA of the cyclone family) and an intermediate buffer (131 kwords IDT FIFO). The aim of the mezzanine is to provide a common interface between the front-end of the various subdetectors and the overall DAQ system. The physical form of the mezzanine (compact $6 \times 6 \mathrm{~cm}^{2}$ board with two rows of $60 \mathrm{I} / \mathrm{O}$ pins each) has been chosen for the ease of integration.

The mezzanines are designed to interface and control each subdetector specific front-end electronics, to sort the data to the event builder, to handle monitoring and slow control from the Global Manager through Ethernet. They handle the following tasks: sequencing of the readout (clocks distribution, R/O registers, digital I/O), local data processing (pedestals subtraction, zero suppression, event validation through external trigger if requested), event time stamping (within $10 \mathrm{~ns}$ steps synchronized with the global distributed clock), data formatting and storage into the FIFO, data transfer and slow control interface.

The data sharing between the FPGA and the processor is performed via IRQ generation either on a PPS basis or on an adjustable threshold on the FIFO. The FPGA configuration can be changed via Ethernet through the micro-processor. The overall mezzanine can be reset via the clock signal received for the synchronization. More information on the Ethernet distributed DAQ core system can be found in $[63,64]$.

The mezzanines are plugged on controller boards specific to each subdetector that could be roughly described as ADC boards for the PMT charge readout in the TT, TDC boards for the drift tubes in the spectrometer and pattern readout boards for the RPCs. They include the front-end controller (readout, configuration and monitoring), the above-mentioned mezzanine, the clock unit (clock decoding, propagation delay measurements) and the power supply unit. Figure 34 and 35 show the schematics of a generic controller board and of its implementations.

The overall DAQ system includes around 1200 sensors (992 for TT, 105 for PT, 50 for RPC, 16 for VETO) for a total of 105,000 readout channels. Each sensor is externally seen as a node on the Ethernet network and receives a common clock signal for synchronization of its local clock. The global DAQ network is divided into two parts, a standard Ethernet network and a clock distribution system.

The Ethernet network includes all sensors, event building servers, general servers (DHCP, NFS, naming service, VNC) and a database cluster. The network is divided into various VLAN to separate and secure the data traffic. Each node is connected to level 2 100/1000 Mbps switches CISCO 2950/60, which are managed via a level 3 1Gbps switch CISCO 3750 acting as the head of network.

The clock distribution system is a specific link between each sensor and the Central Clock Unit synchronized on the GPS. A maximal efficiency is required for this distribution line that can be used for reset or reboot procedures in case of access problems on a controller board. A characteristic feature is the bi-directionality of the system which allows the control of the signal reception and the measurement of the propagation time with the acknowledge signals. 


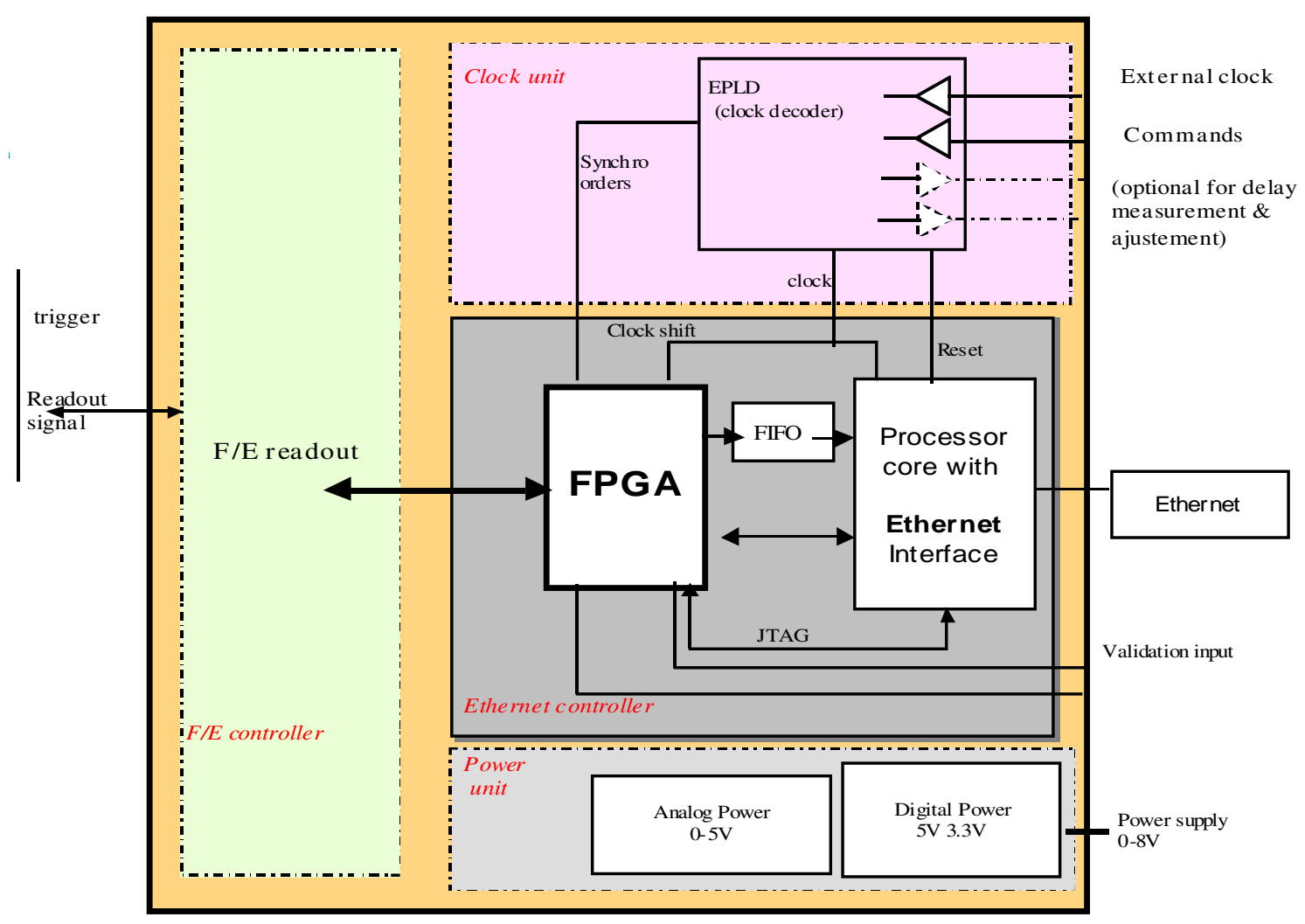

Figure 34. Generic controller board schematics.

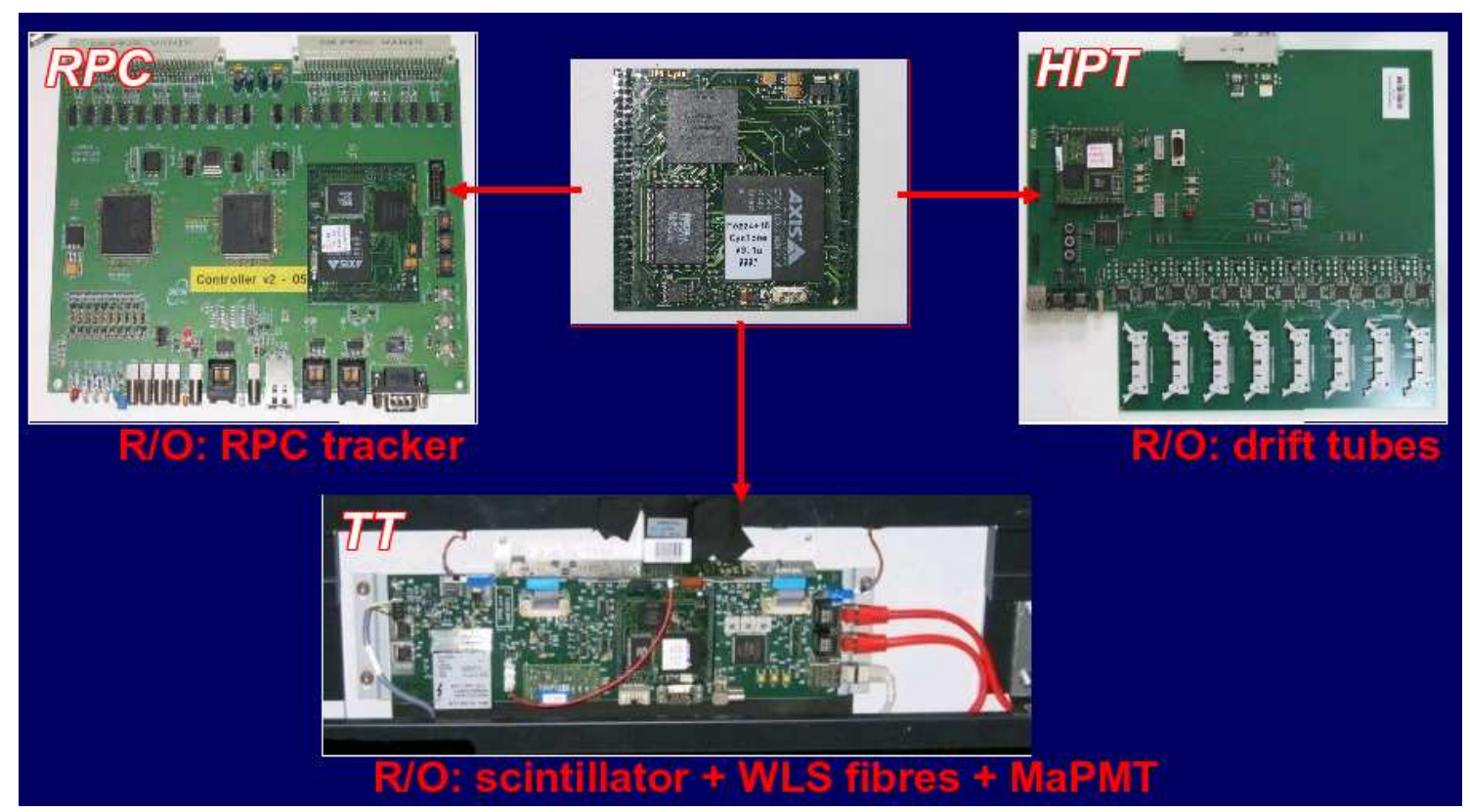

Figure 35. Pictures of the electronic boards. The central board is the mezzanine plugged onto the controller boards (RPC, TT and PT from left to right, respectively). 
The Central Clock unit is a specially developed PCI board which decodes the GPS signal from the external antenna and sends a common clock from a precise oscillator via an optical link. The signal is then converted into electrical format and distributed to the "clock master cards" through M-LVDS bus. Each of these cards deserializes the commands and the clock, and distributes both of them to the clock unit of each controller board through another M-LVDS bus. The Clock Receiver unit can be addressed either individually or in broadcast mode. It receives the control word, decodes it and generates a pulse on the appropriate control signal.

\subsection{Event building and online software}

Each trigger recorded by a sensor (L0 trigger) is timestamped by latching the value of the local fast counter, $100 \mathrm{MHz}$ generated from the common clock via a Phase Locked Loop (PLL). This fine timestamp is added to the absolute time of a PPS-like signal generated by the Central Clock unit, called cycle, after correction of the propagation delay: $T_{\text {event }}=T_{\mathrm{UTC}}+T_{\text {fastcounter }}$.

All hits recorded during a cycle are sent by the sensors to the subdetector corresponding event builder (1 per each SM for the TT, the RPC and the PT, and 1 for VETO) which performs a time sorting, searches for coincidences within a sliding and adjustable gate, applies L1 filters and buffers the data before transmission to the Global Manager. With an overview of all detectors, the Manager performs the overall event building after L2 filtering and records the events. The database used is a ORACLE10g cluster of two servers in direct connection to a disk crate (11 TB capacity).

The distributed client/server software is based on the CORBA (Common Object Request Broker Architecture) standard which is a well established Object Oriented application. It offers a global framework which links the applications running on the sensors, on the Event building servers and on the Manager. The feature of CORBA is the possibility to give each object a reference on a virtual bus (the ORB) so that it can be used by any connected application which gets this reference. All entities connected on the network are registered by a Naming Service.

The online software connects all applications which are classified into three categories: sensor (L0), subdetector DAQ (L1), Manager (L2). The last two offer a G.U.I. designed in the GTKmm framework.

\subsection{UTC time synchronization}

The events recorded by the OPERA DAQ are correlated offline with the CNGS beam by comparing their coordinated universal time (UTC timestamp) with the one of the proton extraction from the SPS. The system includes several parts, which were individually calibrated. At CERN the current pulse of the kicker magnet used for the beam extraction from the SPS to the TT41 line was timetagged with a Symmetricom ${ }^{17}$ XL-DC GPS unit disciplined with a Rubidium oscillator. All cable delays from the antenna and the kicker to the GPS unit were accurately calibrated. The GPS unit was also characterized by the Swiss metrology institute METAS ${ }^{18}$ in order to get an absolute UTC calibration. The UTC time of each proton extraction is recorded in the CNGS database and is accessed by the OPERA offline software. A recent improvement of this system has implied moving the protons time-tag to the level of the BCT detectors (see section 2).

\footnotetext{
${ }^{17}$ Symmetricom Corporate, 2300 Orchard Parkway, San Jose, California 95131 USA.

${ }^{18}$ Federal Office of Metrology METAS, Lindenweg 50, CH-3003 Bern-Wabern, Switzerland.
} 
The path traveled by the protons down to the CNGS target and the distance between the target and OPERA were measured by the CERN geodesy service. The total length from the kicker magnet down to the OPERA detector amounts to $731.5 \mathrm{~km}$ and it is known with $50 \mathrm{~m}$ accuracy. The total time of flight from the kicker is evaluated assuming all particles traveling at the speed of light.

In the external laboratory of LNGS a double ESAT ${ }^{19}$ GPS system disciplined with Rubidium oscillators provides the UTC timing signal to all the underground experiments. The GPS system has an accuracy comparable to the CERN one. In March 2006 the CERN GPS system was moved to LNGS and over a period of more than one month the UTC times of the two systems were intercalibrated every second by using a time interval counter with $300 \mathrm{ps}$ accuracy. A constant offset of $350 \mathrm{~ns}$ was measured among the two systems. The time difference was oscillating around this constant mean value within a band of \pm 23 ns. This result was confirmed in 2007 by a direct comparison of the two systems in their own locations performed by using a transportable reference from a portable Cs atomic clock. The phase of the CERN system was compared with respect to this portable clock, which was then brought to LNGS by car to perform a similar measurement. The trip lasted about 12 hours which represents a period short enough to guarantee the stability of the Cs clock at the level of a few ns.

The LNGS timing signal is sent every ms through a system of optical fibers to the underground experiments. OPERA developed a customized slave clock based on a high stability quartz oscillator which is completely compatible with the LNGS timing format and it is used to dispatch the timing to all the DAQ nodes of the experiment (see also section 7.1) with a $10 \mathrm{~ns}$ period. All the conversion, propagation and processing delays, for a total of about $41 \mathrm{~ms}$, going from the external GPS unit to the output of the OPERA slave clock were calibrated at the ns level by using a double path time comparison technique. A second measurement, which confirmed the former calibration, was performed by using a portable $\mathrm{Cs}$ atomic clock and comparing the time phase of the distributed timing signal in various points of the chain with respect to the absolute UTC Cs reference. Finally, all the DAQ time distribution and processing delays from the slave clocks to the DAQ nodes were characterized at ns accuracy. The DAQ nodes are able to perform a relative self-calibration for the different cable connection lengths so that the DAQ time distribution chain can be described by a single delay of about $4.24 \mathrm{~ms}$.

Taking into account all the calibrations, the time synchronization accuracy between the CERN beam GPS tagging and the OPERA timing system is about $100 \mathrm{~ns}$. This accuracy is largely sufficient to correlate the events with the beam. As mentioned in section 2, the CNGS timing structure is such that, for each CNGS cycle, protons are extracted from the SPS in two spills lasting $10.5 \mu \mathrm{s}$ each and separated by $50 \mathrm{~ms}$. The offline program correlating the events with the beam subtracts from the OPERA UTC time stamp of the events all the delays due to the particles time of flight and the calibration of the various components of the chain and selects events where the difference of the OPERA and CNGS time stamps is within a window of $20 \mu \mathrm{s}$.

\subsection{Offline software}

In OPERA, persistent data both for the electronic detectors (see section 7.2) and for the scanned emulsions (see section 8.2) are recorded in ORACLE databases (DB). The offline computing frame-

\footnotetext{
${ }^{19}$ ESAT Srl, Viale Antonio Gramsci, 366020 Paglieta (Chieti), Italy.
} 
work is a set of C++ packages running mainly on Linux platforms, managed by CMT [65] and stored in CERN-based CVS repositories. ROOT [66] is used extensively for data retrieval from the DB managed by TTrees in binary files. It is also used for the geometrical description of the detector and as an interface with the Monte Carlo (GEANT 3.14 [67]) through the VMC package [68].

Full simulation of the detector response is available for a wide class of events including neutrino interactions from CNGS in the active detector area, interactions in the surrounding material (e.g. in rock or in the Borexino experiment located just in front of OPERA) and cosmic rays.

\section{Emulsion handling and analysis}

\subsection{Brick extraction and film development}

Once a neutrino interaction is reconstructed in the electronic detectors, the brick most probably containing the interaction vertex is identified by dedicated offline algorithms, extracted by the BMS system and exposed to X-rays. The X-ray gun produces five circular (150 $\mu \mathrm{m}$ diameter) spots on both the CS films and the most downstream film of the brick. These spots are meant for the precise alignment between the two films of the CS-doublet and for the relative alignment between the CSdoublet and the brick. Then, the CS-doublet is detached from the brick and brought to the CS handling facility while the brick itself is stored in Hall B in a room surrounded by a $5 \mathrm{~cm}$ thick iron shielding. This shielding was designed after a radioactivity survey on site. It reduces the low energy background from the concrete by a factor of 20 in the region of the ${ }^{40} \mathrm{~K}(1460.7 \mathrm{keV})$ peak.

The CS facility is also located in Hall B and is organized as a double safe-light photographic darkroom equipped for emulsion treatment. The CS envelope is opened, the films are separated and labeled with a matrix code and a plate number using a computer-driven optical device. After labeling, the chemical processing starts and lasts about three hours per batch. The facility is equipped to develop up to $60 \mathrm{CS}$-doublets per day.

After the scanning of the CS-doublet, if no tracks are found, a new CS-doublet is attached to the brick, which is put back in the target. It may also be decided to continue the event search and extract one or more of the neighbouring bricks. If the result of the CS scanning is positive, the brick is exposed to X-rays to print lateral marks used for quick inter-calibration of the films. This $\mathrm{X}$-ray exposure is also performed underground.

The next brick handling steps are the cosmic-ray exposure, the brick dismantling, the film development, drying and packing for shipment to the scanning laboratories. All these activities are performed in a building of the LNGS external laboratory. This building hosts a chemical plant on the ground floor, a darkroom on the underground floor and the cosmic-ray pit in the basement. The pit is a cylinder of $3.0 \mathrm{~m}$ diameter located at a depth of $8.25 \mathrm{~m}$ below the ground level of the LNGS external laboratory. It is linked to the main building by a $10 \mathrm{~m}$ long corridor. The bricks are exposed with films horizontal on a stage shielded on the top with a $40 \mathrm{~cm}$ thick iron slab. A shielding structure made of iron and plastic slabs is used to absorb soft radiation due to interactions of electrons and neutrons. The cosmic-ray exposure time is set to about 12 hours, corresponding to about one penetrating track per $\mathrm{mm}^{2}$ in the angular acceptance relevant for the scanning. The bricks are transported individually from the underground to the surface laboratory in special boxes designed to avoid mechanical shocks and prevent plate misalignment. 


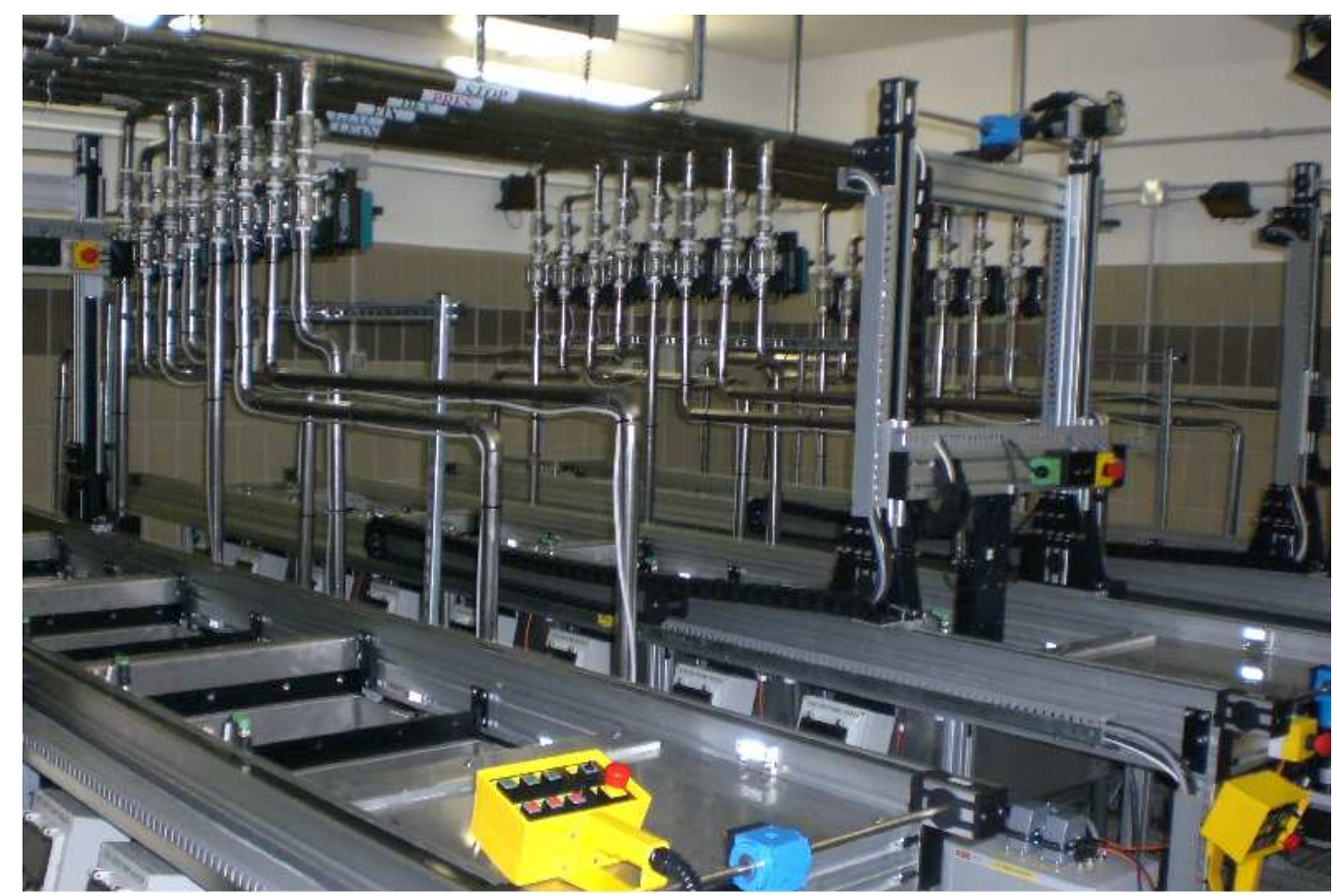

Figure 36. Two chains of the film development facility at the LNGS external laboratory.

The darkroom is a large area (about $130 \mathrm{~m}^{2}$ ) divided in three parts for brick unpacking and labeling, film development and drying. The brick is first opened, by removing the aluminum spider and the plastic protection. The unpacking is performed with dedicated semi-automatic tools. Piling defects that occurred occasionally during the brick assembly procedure (e.g. double emulsion or lead layers, or damaged emulsions) are recorded during this unpacking phase. A label put by light exposure identifies uniquely each film. Then, the films are inserted into holders and dispatched to the developing plant in bunches of six bricks.

The large chemical development plant is fully automated and able to handle up to 3000 films per day. The full development procedure is accomplished by six automated chains connected to a series of tanks that contain the chemical solutions, as shown in figure 36. Movable arms under PLC computer control allow to displace and insert the plate holders into or out of each tank at the scheduled time. The other tasks of the system are the exhaustion of chemical waste and the insertion of fresh ones. Commercial up-to-date technology (UNILINE Roll-On technology) was adopted for the long belt chains, and high quality modular elements were selected for the mechanical structure. A specially designed arch structure is holding a jaw to pick-up and move the film holders. The tanks containing the chemicals are made of high-quality stainless steel with fully chemical-proof soldering. Chemical solutions are prepared by an industrial-type plant and thermalized, ready to be fed to individual tanks in the automated chains through dedicated pipelines.

After development, the films are kept in a humidity controlled room for 48 hours, allowing them to dry with minimal distortion. They are finally packed in polyethylene folders and shipped to the European and Japanese laboratories for scanning. 


\subsection{Emulsion scanning systems}

As mentioned in section 1, in the early 90's the development of fully automated scanning microscopes brought the emulsion technique to a revival. The first fully automatic system was developed in Japan [69] and, since then, the speed of the scanning systems has steadily improved, taking advantage of the developments in electronics and digital image processing [70]. The expected rate of 30 events per day (under nominal CNGS running conditions) and the average emulsion surface to be scanned per event (about $200 \mathrm{~cm}^{2}$ ) required for OPERA a dedicated $R \& D$ project. This lead to the development of two scanning systems with comparable performance, although the implementation differs both in the hardware and software architecture.

The European Scanning System (ESS), the result of a joint effort of several European laboratories, is shown in the left part of figure 37. It makes use of commercial subsystems in a software-based framework; therefore it can be easily updated when more performing components appear on the market. The ESS, derived from a system developed in the late 90's [71] in Salerno, is extensively described in refs. [72-74]. The microscope is a Cartesian robot, holding the emulsion film on a horizontal stage movable in X-Y coordinates, with a CMOS camera mounted on the optical axis (Z), along which it can be moved to change the focal plane with a step roughly equal to the focal depth of about $3 \mu \mathrm{m}$. The control workstation hosts a motion control unit that directs the stage to span the area to be scanned and drives the camera along the $\mathrm{Z}$ axis to produce optical tomographic image sequences (with the $\mathrm{X}$-Y stage holding steady). Areas larger than a single field of view $\left(\sim 300 \times 400 \mu \mathrm{m}^{2}\right)$ are scanned by repeating the data acquisition sequence on a grid of adjacent fields of view. The stage is moved to the desired position and the images are grabbed after it stops, with a stop-and-go algorithm.

The images, grabbed by a Megapixel camera at the speed of 376 frames per second while the camera is moving in the $\mathrm{Z}$ direction, are almost still thanks to an electronic shutter (1/6097s). They are sent to a vision processing board hosted in the control workstation to suppress the noise. This process enhances the images of developed grains, thus reducing the effect of residual optical aberrations for far-off-axis objects. Three-dimensional sequences of aligned clusters (grains) are then recognized and reconstructed on the fly by the CPUs of the host workstation: a reconstructed cluster sequence in an emulsion layer is called micro-track and it is made of at least 6 and at most 16 clusters. Raw data thus produced are essentially micro-tracks together with their grains; in each laboratory, they are sent across a LAN network to a local computing cluster for further postprocessing and data quality checking, eventually being written to a local DB server. The linking of two matching micro-tracks of an emulsion plate produces the so-called base-track. The whole system can work at a sustained speed of $20 \mathrm{~cm}^{2} /$ h/layer, 24 hours/day, with an average data rate as large as $4 \mathrm{~GB} /$ day/microscope. A different setup of this system makes no use of immersion oil as interface between the objective lens and the film being scanned [75]. This option is currently being used in some of the emulsion laboratories with a dedicated automated film changer.

A different system, named S-UTS (Super-Ultra Track Selector), has been developed in Nagoya and is based on highly customized components. A picture of this system is shown in the right part of figure 37. The main feature of this approach is the removal of the stop-and-go process of the stage in the image data taking, which is the mechanical bottle-neck of traditional systems. To avoid the stop, the objective lens moves at the same constant speed of the stage while moving also along 

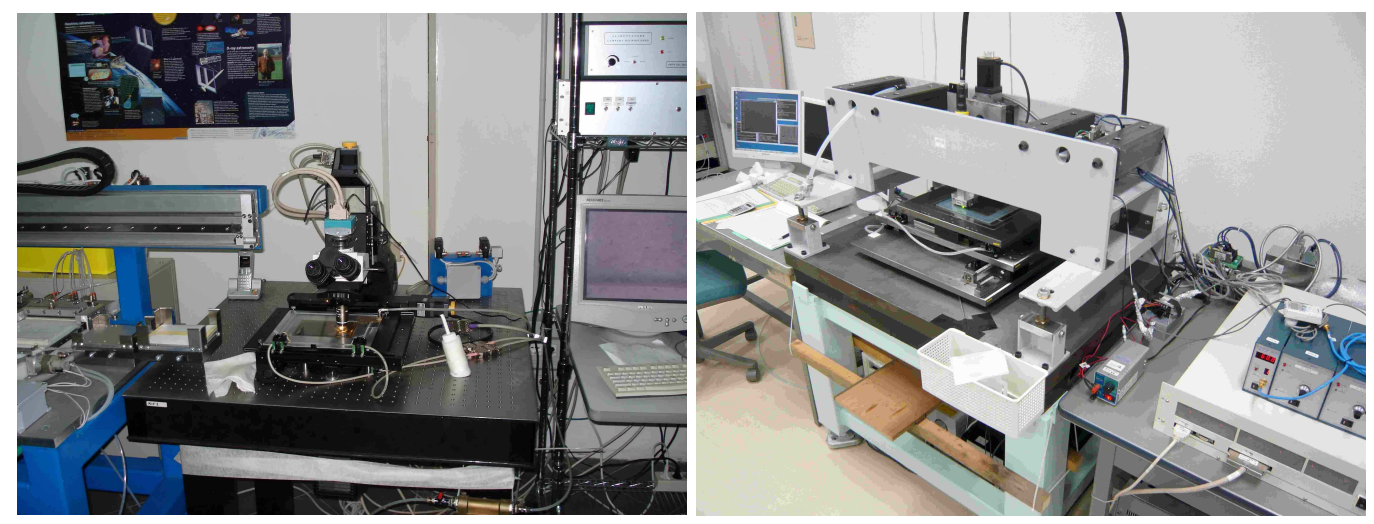

Figure 37. A photograph of the European (left) and Japanese (right) scanning system.

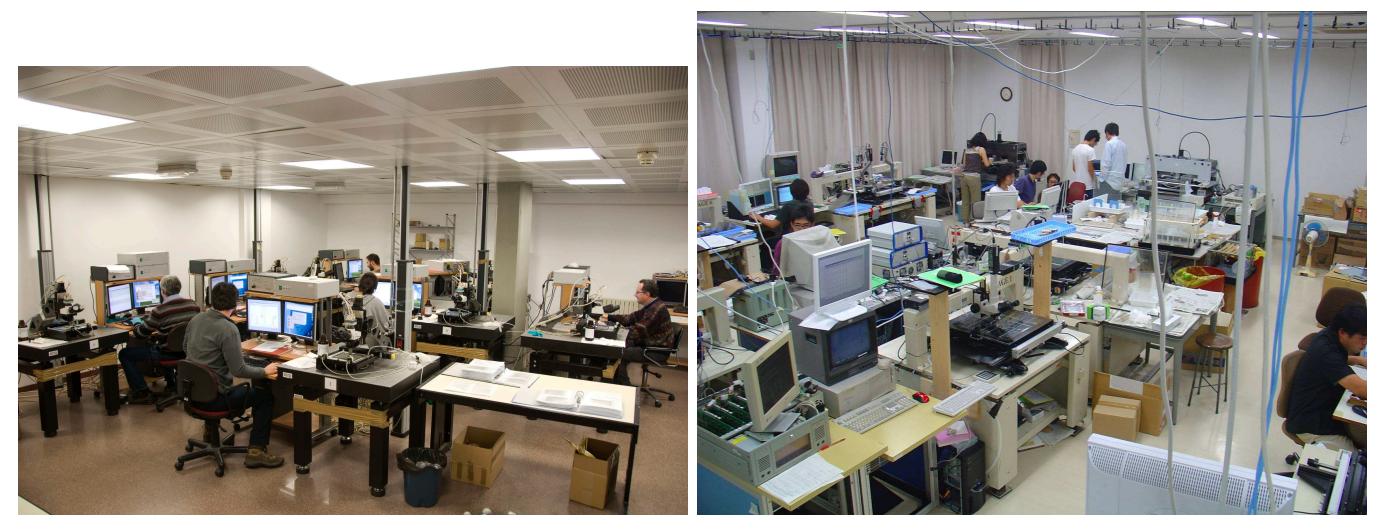

Figure 38. The European (left) and Japanese (right) CS Scanning Stations.

the vertical axis and grabbing images with a very fast CCD camera running at $3000 \mathrm{~Hz}$. The optical system is moved by a piezo-electric device. The camera has a sensor with $512 \times 512$ pixels, which imposes a smaller field of view $\left(\sim 120 \times 120 \mu \mathrm{m}^{2}\right)$ to guarantee a comparable position resolution (about $0.3 \mu \mathrm{m} /$ pixel). The very high speed camera provides a data rate of about $1.3 \mathrm{~GB} / \mathrm{s}$. This is handled by a front-end image processor which makes the zero-suppression and the pixel packing, reducing the rate to $150 \div 300 \mathrm{MB} / \mathrm{s}$. A dedicated processing board makes the track recognition, building micro-tracks and storing them in a temporary storage device with a rate of $2 \div 10 \mathrm{MB} / \mathrm{s}$. A computer processes the micro-tracks, linking the micro-tracks of different layers and writing the resulting tracks in a database which is used as input for physics analysis. The routine scanning speed is $20 \mathrm{~cm}^{2} / \mathrm{h} /$ layer while one of the S-UTS systems has reached the speed of $72 \mathrm{~cm}^{2} / \mathrm{h} /$ layer by using a larger field of view.

All data are stored and shared throughout the collaboration by collecting them in a Central DB, existing in two identical master copies at LNGS (Italy) and in the IN2P3 Computing Center in Lyon (France). 

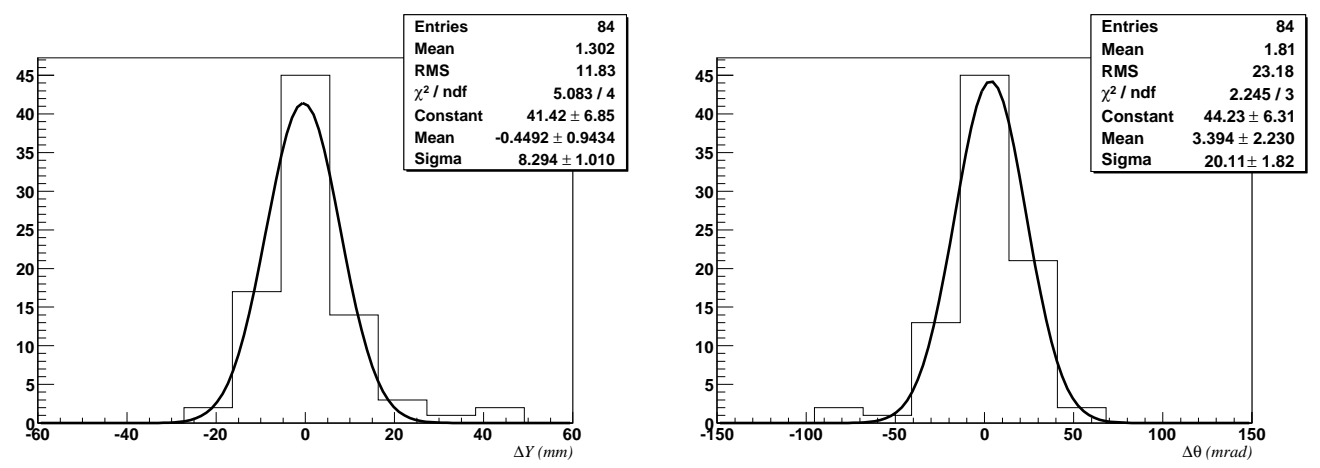

Figure 39. Distribution of the position (left) and angular (right) residuals between the muon prediction and the track measured in the CS films.
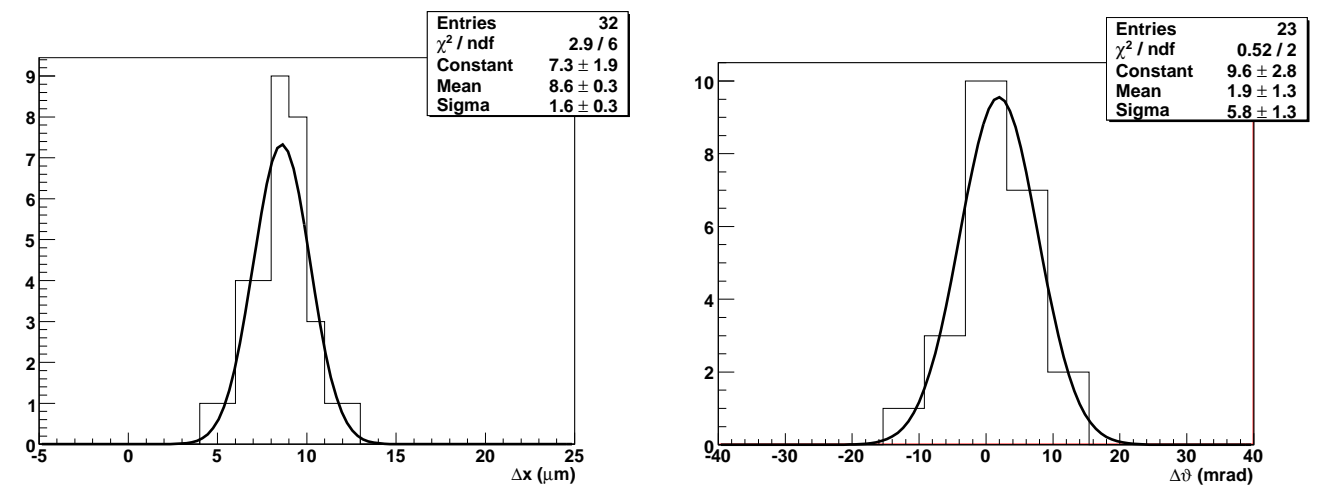

Figure 40. Distribution of the position (left) and angular (right) residuals between the base-tracks measured in two CS films.

\section{Emulsion scanning methods}

There are two different ways of scanning the emulsions, corresponding to different needs. The first is the so-called general scanning that looks for tracks in all the available angular range (typically $\pm 400 \mathrm{mrad}$ around the vertical direction). This method is used when there is no a-priori knowledge of the track angle. In the case the track angle is actually estimated, a limited angular range is used which makes the scanning faster and the tracking slightly more efficient. Here in the following we describe the main analysis stream of the scanning of the events.

The scanning of the CS is done in two laboratories hosting a farm of microscopes (Scanning Stations), one at LNGS and the other in Nagoya. Figure 38 shows a photograph of one of them. Electronic detectors predict the brick where the neutrino interaction occurred. Moreover, for events with a muon in the final state, a prediction for the slope of the muon and its impact on the brick is also given with an accuracy shown in the left and right plot of figure 39. For NC events, the averaged center of TT hits provides the center of the scanning area, with an accuracy similar to that of the muon. This makes the scanning area on the Changeable Sheets as wide as $50 \mathrm{~cm}^{2}$. The scanning is done independently on both CS, in order to maximize the efficiency.

The agreement between the two reconstructed tracks in the CS doublet is quite stringent, given the precise alignment provided by the X-ray exposure as described in section 3.2. Figure 40 shows 

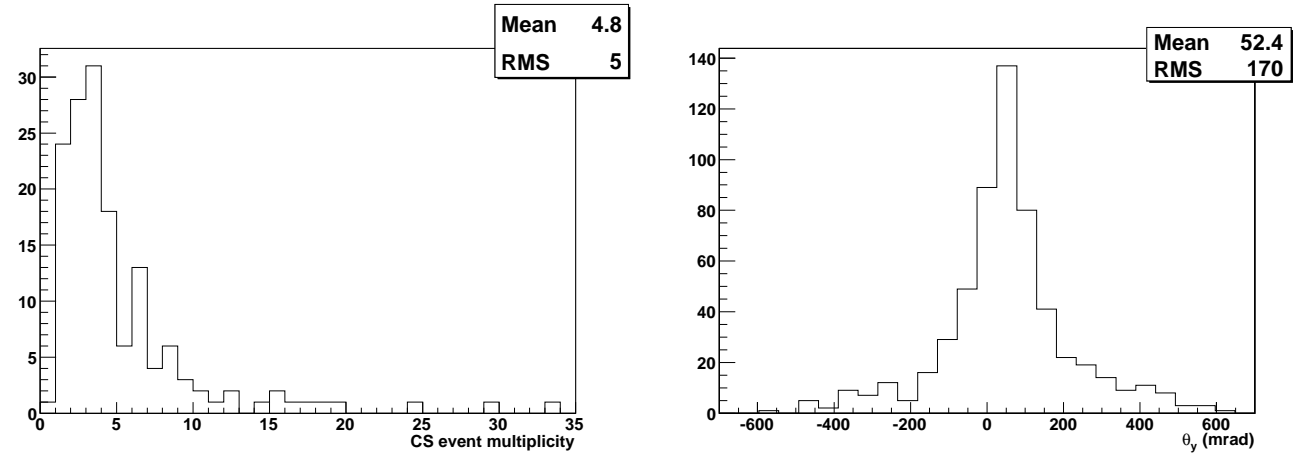

Figure 41. Distribution of the track multiplicity at the CS level (left). Distribution of the Y (vertical) projection angle of the tracks measured in the CS scanning (right).

the distribution of the position and the angular residuals in the matching of tracks in the doublet. The intrinsic position accuracy is less than $2 \mu \mathrm{m}$ while the residual is dominated by the systematic uncertainty in the mark measurement which is of the order of $10 \mu \mathrm{m}$. All candidate tracks are then validated by an eye inspection to reject the small remnant background due to accidental coincidences. The tolerances between the two base-tracks are set accordingly: $40 \mu \mathrm{m}$ in position and $10 \mathrm{mrad}$ in angle, the latter enlarged according to the track slope.

The tracking efficiency has been measured on the double-refreshed CS films by using tracks produced in neutrino interactions and hence it is averaged on the relevant angular spectrum. The result shows a reconstruction efficiency of $86 \%$ at the level of single base-tracks. This implies a particle trajectory reconstruction efficiency of $75 \%$ when double base-tracks are used.

Using Compton electrons from environmental radioactivity, the systematic uncertainties in the alignment between the two CS films are reduced, bringing the position accuracy at the level of 1 $\mu \mathrm{m}$ [35]. Given the larger background in the micro-track reconstruction, such an accuracy allows for the application of tighter cuts and thus it makes the use of micro-tracks in the track formation possible. If no double base-track candidate is found, after the application of the fine alignment procedure, candidates made of 3 micro-tracks are selected, thus increasing the particle trajectory reconstruction efficiency to $90 \%$.

The scanning of a CS doublet typically takes several hours. The left plot of figure 41 shows the distribution of the track multiplicity at the CS level, and the right plot shows the distribution of the vertical projection angle of the tracks measured in the CS scanning. A tilt of $52 \mathrm{mrad}$ is visible, in good agreement with the CNGS beam slope.

All track candidates seen on the CS are extrapolated back to the target brick, $4.5 \mathrm{~mm}$ upstream, and searched for in the most downstream film of the brick. The set of X-ray marks on the CS doublet are on purpose visible also on the most downstream film. This ensures an accuracy in position of about $70 \mu \mathrm{m}$ and less than $10 \mathrm{mrad}$ in angle as shown in figure 42 . Systematic uncertainties dominate the residuals.

The tracks validated on the downstream film are then followed from film to film until they disappear: this phase is called scan-back. The track following requires a precise alignment between consecutive films while the mechanical accuracy of the brick assembly machine is a few hundred microns. Lateral X-ray marks are printed on the brick as described in section 8.1. This reference 

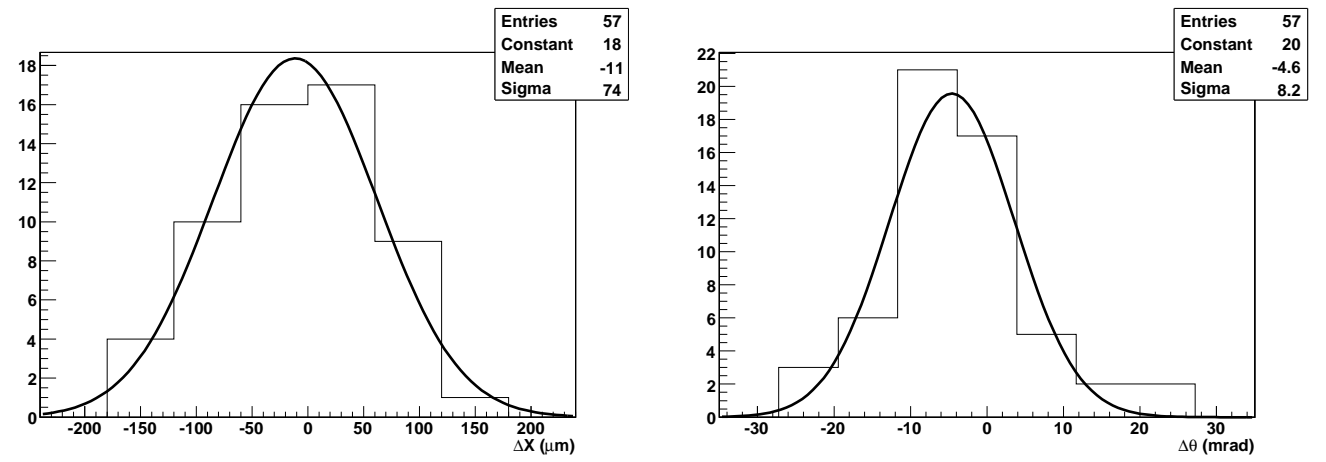

Figure 42. Distribution of the position (left) and angular (right) residuals between the track measured in the CS doublet and the one found in the most downstream film of the brick.
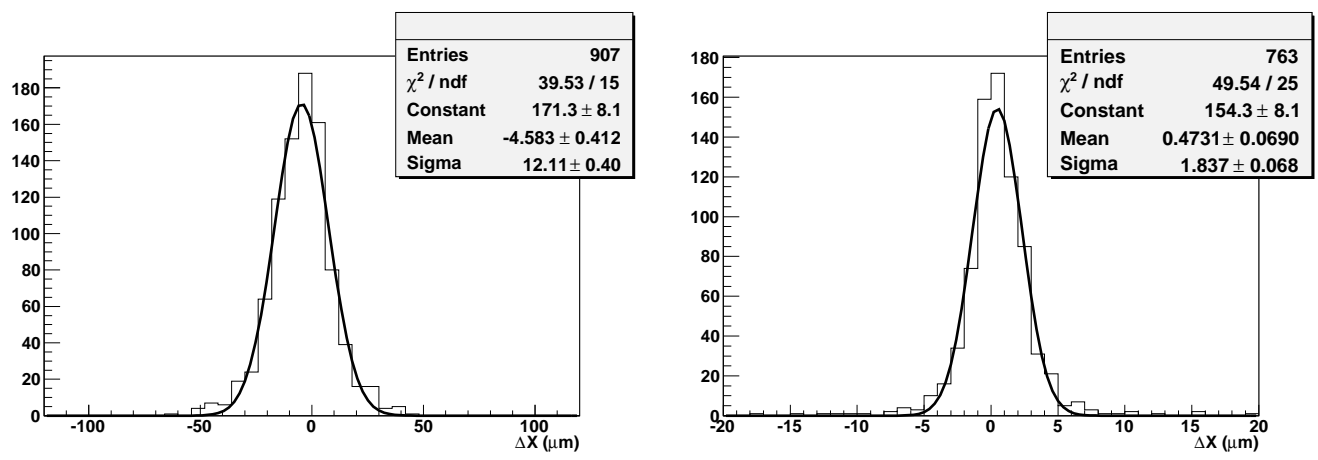

Figure 43. Distribution of the position residuals obtained in the track follow-up with the film-to-film alignment performed with lateral X-ray marks (left) and cosmic rays (right).

provides an inter-calibration accuracy of about $12 \mu \mathrm{m}$ as shown in the left plot of figure 43 . This accuracy is sufficient for a fast scan-back, since the track is searched for within just one microscope view. The stopping of a track is defined by its disappearance in three consecutive films.

The ECC bricks are also exposed to cosmic rays at the surface before disassembling (see section 8.1). By using the cosmic rays in a few $\mathrm{mm}^{2}$ around the track, a position accuracy of better than $2 \mu \mathrm{m}$ can be obtained, as shown in the right plot of figure 43 . This precise alignment is important for the physics analysis, like the small kink detection or the momentum measurement using Multiple Coulomb Scattering with an accuracy of better than $20 \%$ at $2 \mathrm{GeV}$ and $40 \%$ at $1 \mathrm{GeV}$.

A track stop can be either due to a primary or to a secondary vertex. In order to study the stopping point, a volume made of $1 \mathrm{~cm}^{2}$ on 5 films downstream and 5 films upstream of the stopping plate is scanned around each stopping point. The volume is skewed according to the neutrino beam slope, to allow for a complete containment of all products of a possible interaction. The data are processed by an off-line program to reconstruct all the tracks originating inside the volume: these tracks are input for a vertex reconstruction algorithm which is tuned to find also decay topologies. Figure 44 shows the different steps of the emulsion data processing: on the left all base-tracks in the 11 films of the volume are reconstructed; they participate to the alignment process from which tracks are reconstructed as shown in the middle plot; on the right passing-through tracks are discarded and the vertexing algorithm reconstructs the vertex. 

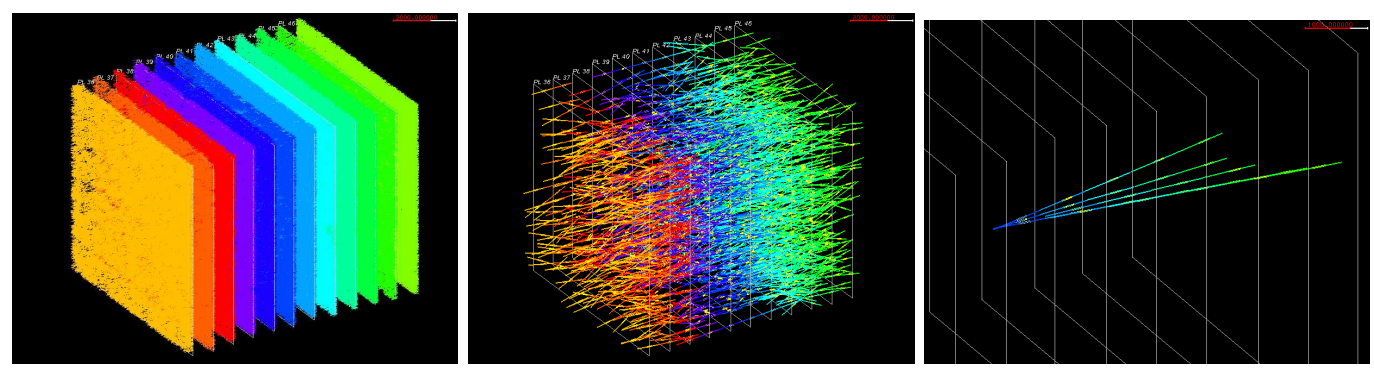

Figure 44. Different steps of the emulsion data processing.
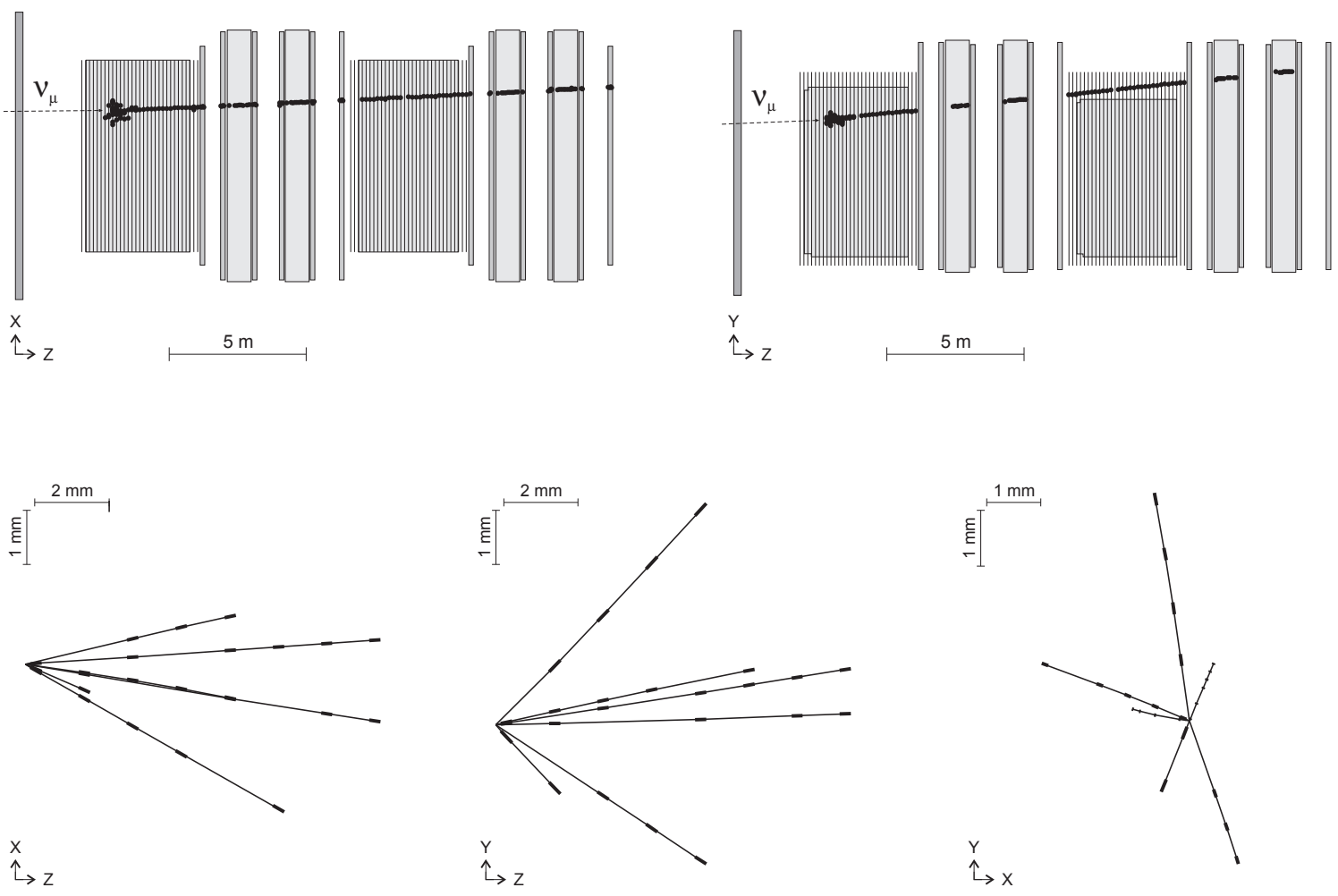

Figure 45. Event display of a CC event as reconstructed in the electronic detectors (top) and in the emulsions (bottom). The electronic detectors are shown in top view on the left and in side view on the right. At the bottom the reconstructed primary vertex is shown in top view $(\mathrm{X}-\mathrm{Z})$, side view (Y-Z) and front view $(\mathrm{X}-\mathrm{Y})$.

The top of figure 45 shows an event display as reconstructed in the electronic detectors (top view on the left and side view on the right); in the bottom part there are the top view (X-Z), the side view (Y-Z) and the front view (X-Y) of the reconstructed primary vertex in the emulsions.

The tracks attached to the primary vertex are followed downstream, with a procedure analogous to the scan-back but in the opposite direction, to check whether they show any decay topology and measure their momentum. 


\section{Conclusions}

The OPERA neutrino oscillation experiment is designed to perform the most direct test of the oscillation phenomenon through the detection of $v_{\tau}$ appearance in the CERN to Gran Sasso (CNGS) neutrino beam. It took from Summer 2003 to Spring 2008 to build the detector with its ancillary infrastructure in the LNGS underground laboratory. This paper reviewed the design, construction and technical performance of all the subsystems of OPERA, i.e. the lead-emulsion target bricks, the facilities for their mass production and handling, the real-time electronic trackers and muon spectrometers and the automatic scanning microscopes. We also presented the full analysis chain from the data acquisition with the electronic detectors up to the development and scanning of the emulsions. During the 2008 CNGS run, OPERA collected about 1700 neutrino interactions in the target bricks, which are presently under analysis.

\section{Acknowledgments}

We wish to express our gratitude to CERN for the successful commissioning and operation of CNGS and to INFN for providing all the needed infrastructures and the support of the LNGS staff and for providing - as host Institution - fellowships and grants to non-Italian researchers during their stays at LNGS. In particular, we thank the Directorate, the Technical and General Services Division, the Prevention and Protection Service, the Computing and Network Service, the Administration and all the experiment support Staff of LNGS for the continuous help to OPERA during the construction, installation and commissioning phases. We gratefully acknowledge the outstanding contribution of the Technical Staff of our collaborating institutions: P. De Harenne, R. Gindroz, S. Hannaert, M. Pins, R. Pins (Brussels Univ.), L. Berger, S. Fleig, H.J. Ohmacht, J. Schuett, A. Spikowsky, R. van Staa (Hamburg Univ.), P.Y. Jallud (IN2P3 IPNL), C. Schwab, H. Kocher (IN2P3 IRES), L. Giacobone, J. Jacquemier, F. Peltier (IN2P3 LAPP), A. Andriani, P. Dipinto, V. Di Pinto (INFN Bari), E. Bottazzi, L. Degli Esposti, P. Calligola, V. Togo, C. Valieri (INFN Bologna), the Technical staff of INFN-LNF: C. Fusco, F. Iungo, M.F. Sardone (LNF Accelerator Division), A. Balla, G. Corradi, U. Denni, A. Frani, M. Gatta, G. Paoluzzi, G. Papalino (Servizio elettronica e Automazione), A. Cecchetti, D. Orecchini (Servizio Progettazione Apparati Sperimentali), M. Arpaia, G. Catitti, A. Ceccarelli, N. Intaglietta, A. Tiburzi (Servizio Sviluppo e Costruzione Rivelatori), A. Gambarara, A. Mengucci, T. Tonto, M. Ventura (Staff tecnico di Esperimento); we thank the Technical Staff of INFN-LNGS: A. Candela, M. De Deo, M. D'incecco, M. Lindozzi (Servizio Elettronica), A. Corsi, E. Tatananni (Officina Meccanica), M. Balata, L. Ioannucci (Servizio Chimica e Impianti Chimici); the Technical Staff of INFN-Naples: C. Cassese, F. Cassese, B. D'Aquino, B. De Fazio, R. Rocco (Servizio Officina), G. Passeggio, E. Vanzanella (Servizio Progettazione), A. Attanasio, V. Masone, P. Parascandolo, R. Chiaramonte (Servizio Elettronica). We also thank E. Borsato, L. Castellani, A. Benato, R. Peghin (INFN Padova), A. Buccheri, M. Capodiferro, P. Pecchi, A. Pelosi, A. Ruggieri (INFN Rome), A. Frolov, V. Belevsky, A. Samokhotov, P. Chernov, V. Korolev V. Gusevsky and I. Mitrofanov (ITEP). We acknowledge DESY for providing the infrastructure for the PT mass production and for the support of the DESY Alignment Group. We warmly acknowledge funding from our national agencies: Fonds National de la Recherche Scientifique et Institut Interuniversitaire des Sciences Nucleaires for Belgium, MoSES for Croatia, 
IN2P3-CNRS for France, BMBF for Germany, INFN for Italy, the Japan Society for the Promotion of Science (JSPS), the Ministry of Education, Culture, Sports, Science and Technology (MEXT) and the Promotion and Mutual Aid Corporation for Private Schools of Japan for Japan, SNF and ETH Zurich for Switzerland, the Russian Foundation for Basic Research (grants 06-02-16864, 06-02-16337, 08-02-91005 and 08-02-01086) for Russia, the Korea Research Foundation Grant (KRF-2007-013-C00015) for Korea. We dedicate this paper to the memory of Romolo Diotallevi, an esteemed member of our Collaboration, who highly contributed to the OPERA emulsion development facilities. He was a friend to many of us and we miss him dearly.

\section{References}

[1] SuPER-KAmiOKANDE collaboration, Y. Fukuda et al., Evidence for oscillation of atmospheric neutrinos, Phys. Rev. Lett. 81 (1998) 1562;

SUPER-KAMIOKANDE collaboration, J. Hosaka et al., Three flavor neutrino oscillation analysis of atmospheric neutrinos in Super-Kamiokande, Phys. Rev. D 74 (2006) 032002;

SUPER-KAMIOKANDE collaboration, K. Abe et al., A Measurement of Atmospheric Neutrino Flux Consistent with Tau Neutrino Appearance, Phys. Rev. Lett. 97 (2006) 171801.

[2] KAMIOKANDE-II collaboration, K.S. Hirata et al., Experimental Study of the Atmospheric Neutrino Flux, Phys. Lett. B 205 (1988) 416;

MACRO collaboration, S.P. Ahlen et al., Atmospheric neutrino flux measurement using upgoing muons, Phys. Lett. B 357 (1995) 481;

MACRO collaboration, M. Ambrosio et al., Measurement of the atmospheric neutrino-induced upgoing muon flux using MACRO, Phys. Lett. B 434 (1998) 451;

MACRO collaboration, M. Ambrosio et al., Matter effects in upward-going muons and sterile neutrino oscillations, Phys. Lett. B 517 (2001) 59;

MACRO collaboration, M. Ambrosio et al., Measurements of atmospheric muon neutrino oscillations, global analysis of the data collected with MACRO detector,

Eur. Phys. J. C 36 (2004) 323;

SOUDAN-2 collaboration, W.W.M. Allison et al., The atmospheric neutrino flavor ratio from a 3.9 fiducial kiloton-year exposure of Soudan 2, Phys. Lett. B 449 (1999) 137;

SOUDAN 2 collaboration, M.C. Sanchez et al., Observation of atmospheric neutrino oscillations in Soudan 2, Phys. Rev. D 68 (2003) 113004;

SOUDAN-2 collaboration, W.W.M. Allison et al., Neutrino oscillation effects in Soudan-2 upward-stopping muons, Phys. Rev. D 72 (2005) 052005;

K2K collaboration, M.H. Ahn et al., Measurement of Neutrino Oscillation by the K2K Experiment, Phys. Rev. D 74 (2006) 072003;

MINOS collaboration, D.G. Michael et al., Observation of muon neutrino disappearance with the MINOS detectors and the NuMI neutrino beam, Phys. Rev. Lett. 97 (2006) 191801.

[3] GALLEX collaboration, W. Hampel et al., GALLEX solar neutrino observations: results for GALLEX IV, Phys. Lett. B 447 (1999) 127;

SAGE collaboration, J.N. Abdurashitov et al., Measurement of the solar neutrino capture rate with gallium metal, Phys. Rev. C 60 (1999) 055801;

GNO collaboration, M. Altmann et al., Complete results for five years of GNO solar neutrino observations, Phys. Lett. B 616 (2005) 174;

KAMIOKANDE collaboration, Y. Fukuda et al., Solar neutrino data covering solar cycle 22, Phys. Rev. Lett. 77 (1996) 1683; 
SuPER-KAMKIOKANDE collaboration, J. Hosaka et al., Solar neutrino measurements in Super-Kamiokande-I, Phys. Rev. D 73 (2006) 112001;

SNO collaboration, B. Aharmim et al., Electron energy spectra, fluxes and day-night asymmetries of B-8 solar neutrinos from the 391-day salt phase SNO data set, Phys. Rev. C 72 (2005) 055502.

[4] KAMLAND collaboration, T. Araki et al., Measurement of neutrino oscillation with KamLAND: evidence of spectral distortion, Phys. Rev. Lett. 94 (2005) 081801;

CHOOZ collaboration, M. Apollonio et al., Search for neutrino oscillations on a long base-line at the CHOOZ nuclear power station, Eur. Phys. J. C 27 (2003) 331;

PALO VERDE collaboration, F. Boehm et al., Search for Neutrino Oscillations at the Palo Verde Nuclear Reactors, Phys. Rev. Lett. 84 (2000) 3764.

[5] K. Niwa, Emulsion techniques for short and long-baseline experiments, Contrib. to the "Snowmass '94" Conference on particle and Nuclear Astrophysics and Cosmology in the next Millennium, Snowmass (1994);

A. Ereditato, K. Niwa and P. Strolin, The emulsion technique for short, medium and long baseline $v_{\mu}-v_{\tau}$ oscillation experiments, INFN-AE-97-06, Nagoya DPNU-97-07, 27 January 1997;

A. Ereditato, P.E. Strolin and K. Niwa, OPERA: an emulsion detector for a long baseline $v_{\mu} v / \tau$ oscillation search, Nucl. Phys. Proc. Suppl. 66 (1998) 423.

[6] OPERA collaboration, M. Güler et al., An appearance experiment to search for $v_{\tau}$ oscillations in the CNGS beam: experimental proposal, CERN-SPSC-2000-028.

[7] OPERA collaboration, R. Acquafredda et al., First events from the CNGS neutrino beam detected in the OPERA experiment, New J. Phys. 8 (2006) 303.

[8] G. Acquistapace et al., The CERN neutrino beam to Gran Sasso (NGS): Conceptual technical design, CERN-YELLOW-98-02.

[9] J. Nishimura, Soryusiron Kenkyu, Japan 12 (1956) 24;

C.M.G. Lattes et al., Chacaltaya emulsion chamber experiment, Int. Conf. on Cosmic Rays, Jaipur India, 5 (1963) 326;

M. Asashi et al., Photoprotons from Aluminum, J. Phys. Soc. Japan 17 (1962) 1536;

S.L.C. Barroso et al., Inelasticity distribution of hadron-Pb collisions, estimated by thick lead emulsion chambers at the Pamirs, in Proc. of the XXV ICRC, Durban South Africa (1997); M. Akashi et al., Large scale emulsion chamber experiment at Mt.Fuji, in Proc. of the Cosmic Ray and Particle Physics Conf., Newark U.S.A. (1978);

J.R. Ren et al., Properties of hadron families observed with thick type emulsion chambers at mts. Kanbala and Fuji, Phys. Rev. D 38 (1988) 1417;

$\mathrm{K}$. Niu, Discoveries of naked charmed particles and of lifetime difference among charm species carried out using Compact ECC, First Int. Workshop on Nuclear Emulsion, Nagoya, 12-14 June 1998;

K. Hoshino et al., On the X-particles observed in the cosmic ray jet shower, Contr. Paper at the XIV INt. Cosmic Ray Conf., Munich, 7 (1975) 2442;

K. Hoshino et al., X-Particle Production in $205 \mathrm{GeV/c}$ Proton Interactions,

Prog. Theor. Phys. 53 (1975) 1859;

N. Ushida et al., Observation of a pair decay of short-lived neutral particles produced in 400-GeV/c proton interactions, Lett. Nuovo Cim. 23 (1978) 577;

H. Fuchi et al., Direct observation of a pair decay of neutral charmed particles produced in a 400 GeV/c proton interaction, Phys. Lett. B 85 (1979) 135;

H. Fuchi et al., Direct observation of charmed particle pairs produced in $340 \mathrm{GeV/c}$ negative pion interactions in an emulsion chamber, Lett. Nuovo Cim. 31 (1981) 199. 
[10] K. Niu, E. Mikumo and Y. Maeda, A possible decay in flight of a new type particle, Prog. Theor. Phys. 46 (1971) 1644.

[11] DONUT collaboration, K. Kodama et al., Observation of tau-neutrino interactions, Phys. Lett. B 504 (2001) 218;

K. Kodama et al., Detection and analysis of tau neutrino interactions in DONUT emulsion target, Nucl. Instrum. Meth. A 493 (2002) 45.

[12] M. De Serio et al., High precision measurements with nuclear emulsions using fast automated microscopes, Nucl. Instrum. Meth. A 554 (2005) 247.

[13] G.D. Lellis et al., Momentum measurement by the angular method in the Emulsion Cloud Chamber, Nucl. Instrum. Meth. A 512 (2003) 539.

[14] K. Kodama et al., Momentum measurement of secondary particle by multiple Coulomb scattering with emulsion cloud chamber in DONuT experiment, Nucl. Instrum. Meth. A 574 (2007) 192.

[15] L. Arrabito et al., Electron/pion separation with an emulsion cloud chamber by using a neural network, 2007 JINST 2 P02001.

[16] Borexino collaboration, G. Alimonti et al., Science and technology of Borexino: a real-time detector for low energy solar neutrinos, Astropart. Phys. 16 (2002) 205.

[17] CHORUS collaboration, E. Eskut et al., The CHORUS experiment to search for $v_{\mu} \rightarrow$ nu/tau oscillation, Nucl. Instrum. Meth. A 401 (1997) 7.

[18] See http://proj-cngs.web.cern.ch/proj-cngs/.

[19] M. Meddahi et al., CNGS: Results from Commissioning, PAC07 Albuquerque, USA, CERN-AB-2007-039 (2007).

[20] K. Schirm, The Bending Magnets For The Proton Transfer Line Of CNGS, CERN-AT-2006-23 (2006).

[21] Th. Zickler, Design Report of the QTG Quadrupoles for the CERN CNGS Line, CERN SL-Note-2000-049-MS (2000).

[22] B. Langenbeck, Design Report of the MDG Correctors Magnets, EDMS1 Id 399883.

[23] L. Jensens, Beam Instrumentation for the CNGS Facility, CERN AB-Note-2006-022 (2006).

[24] R. Jones, CNGS Primary and Target Instrumentation, EMDS1 Id 610790.

[25] J. Wenninger, CNGS and Transfer Stability in 2007, CERN-AB-2008-02 (2008).

[26] L. Bruno et al., CNGS Progress Report 2004, CERN-AB-Note-2004-063 CNGS (2004).

[27] E. Gschwendtner et al., First Operational Experience with the CNGS Facility, Nufact07, 6-11 August 2007, Okayama, Japan, AIP Conf. Proc. 981 (2008) 23.

[28] E. Gschwendtner et al., Design and Performance of the CNGS Secondary Beam Line, PAC07 Albuquerque, USA, CERN-AB-2007-46 (2007).

[29] A. Marsili et al., CNGS Muon Monitor, CERN-AB-2008-044-BI (2008).

[30] A.E. Ball et al., CNGS : Effects of possible alignment errors, CERN-EP-2001-037, CERN-SL-2001-016 EA (2001).

[31] E. Gschwendtner et al., CERN Neutrinos to Gran Sasso: First Beam, NSS-IEEE06, San Diego, 2006, Report CERN-AB-2007-005 (2007). 
[32] T. Nakamura et al., The OPERA film: new nuclear emulsion for large-scale, high-precision experiments, Nucl. Instrum. Meth. A 556 (2006) 80.

[33] OPERA collaboration, A. Anokhina et al., Emulsion sheet doublets as interface trackers for the OPERA experiment, 2008 JINST 3 P07005.

[34] N. Ushida et al., Experimental details on lifetime measurements of neutrino-produced charmed particles in a tagged emulsion spectrometer, Nucl. Instrum. Meth. 224 (1984) 50.

[35] S. Miyamoto et al., Sub-micron alignment for nuclear emulsion plates using low energy electrons caused by radioactive isotopes, Nucl. Instrum. Meth. A 575 (2007) 466.

[36] T. Toshito et al., Study of electron identification in a few GeV region by an emulsion cloud chamber, Rev. Sci. Instrum. 74 (2003) 53.

[37] OPERA collaboration, A. Anokhina et al., Study of the effects induced by lead on the emulsion films of the OPERA experiment, 2008 JINST 3 P07002.

[38] T. Adam et al., The OPERA experiment target tracker, Nucl. Instrum. Meth. A 577 (2007) 523.

[39] C. Gustavino et al., Performance of glass RPC operated in avalanche mode, Nucl. Instrum. Meth. A 527 (2004) 471.

[40] C. Gustavino, A. Candela, M. De Deo, M. D'Incecco and R. Moro, Performance of glass RPC operated in streamer mode with four-fold gas mixtures containing SF-6, Nucl. Instrum. Meth. A 517 (2004) 101.

[41] M. Ambrosio et al., The OPERA magnetic spectrometer, IEEE Trans. Nucl. Sci. 51 (2004) 975.

[42] A. Cazes et al., Electromagnetic characterization of the 990-ton gapless magnets for the OPERA experiment, 2007 JINST 2 T03001;

A. Cecchetti et al., Chemical and Magnetic Characterization of the Steels for the OPERA Spectrometers, OPERA Internal Note n.63 (2004).

[43] G. Di Iorio et al., Ballistic techniques for magnetic field calibration of large iron detectors operating in underground areas, Nucl. Phys. Proc. Suppl. 125 (2003) 27;

$\mathrm{M}$. Incurvati and F. Terranova, Modeling skin effect in large magnetized iron detectors, Nucl. Instrum. Meth. A $\mathbf{5 0 0}$ (2003) 441.

[44] M. Alviggi et al., First results of the cosmic rays test of the RPC of the ATLAS muon spectrometer at LHC, Nucl. Instrum. Meth. A 518 (2004) 79.

[45] M. Abbrescia et al., Performance of the first RPC station prototype for the CMS barrel detector, Nucl. Instrum. Meth. A 456 (2000) 103.

[46] BABAR collaboration, B. Aubert et al., The BaBar detector, Nucl. Instrum. Meth. A 479 (2002) 1.

[47] ARGO-YBJ collaboration, G. Aielli et al., Layout and performance of RPCs used in the Argo-YBJ experiment, Nucl. Instrum. Meth. A 562 (2006) 92.

[48] A. Bergnoli et al., Tests of OPERA RPC Detectors, IEEE Trans. Nucl. Sci. 52 (2005) 2963.

[49] A. Bergnoli et al., The quality control tests for the RPCs of the OPERA experiment, Nucl. Instrum. Meth. A 533 (2004) 203.

[50] A. Mengucci, A. Paoloni, M. Spinetti and L. Votano, Gas mixture studies for streamer operation of Resistive Plate Chambers at low rate, Nucl. Instrum. Meth. A 583 (2007) 264.

[51] M. Bazzi et al., The I-meter, a distributor unit for the OPERA RPC HV system, Nucl. Instrum. Meth. A 580 (2007) 1441. 
[52] A. Bergnoli et al., The OPERA spectrometer slow control system, IEEE Trans. Nucl. Sci. 55 (2008) 349.

[53] M. Ambrosio et al., The OPERA muon spectrometer tracking electronics, Nucl. Instrum. Meth. A 533 (2004) 173.

[54] A. Bergnoli et al., The OPERA spectrometer front-end electronics, RT2007-CM-EXIST05, Apr 2007, Presented at 15th IEEE Real Time Conference 2007 (RT 07), Batavia, Illinois, 29 April - 4 May 2007.

[55] G. Corradi et al., A sixsteen channel Timing Board for the OPERA RPCs, OPERA Internal note n.70 (2005).

[56] R. Zimmermann et al., The precision tracker of the OPERA detector, Nucl. Instrum. Meth. A 555 (2005) 435.

[57] T. Ferber et al., The gas system of the drift tube detector of the neutrino experiment OPERA, Nucl. Instrum. Meth. A 592 (2008) 493.

[58] G. Felici, A. Paoloni, R. van Staa and R. Zimmermann, Concept of the Trigger System for the Precision Tracker, OPERA Internal note n.71 (2005).

[59] O. Ross, Winkelaufösung und Spureffizienz am Teststand sowie Aufbau der Hochspannungsversorgung des Driftröhrenspektrometers für das OPERA-Experiment, diploma thesis (in German), (2006).

[60] R. Bosch, GmbH, CAN Specification, V. 2.0, Postfach 300240, D-70442 Stuttgart (1991).

[61] J. Schütt and R. van Staa, OSUB OPERA Support Board, (2005); OTB OPERA Trigger Board, (2006), available at http://wwwiexp.desy.de/groups/teb/pdf/.

[62] see http://developer.axis.com/products/etrax1001x/.

[63] C. Girerd et al, Ethernet network based DAQ and smart sensors for the OPERA long-baseline neutrino experiment, LYCEN 2000-109, IEEE Nuclear Science Symposium and Nuclear Imaging, Lyon, October 15-20 2000.

[64] L. Chaussard et al, The scintillator option for the OPERA target tracker: results on the readout, HAL:in2p3-00010669, 2001, available at http://hal.in2p3.fr/in2p3-00010669/en/.

[65] See http://www.cmtsite.org.

[66] R. Brun and F. Rademakers, ROOT: an object oriented data analysis framework, Nucl. Instrum. Meth. A 389 (1997) 81;

see also http://root.cern.ch/.

[67] R. Brun et al., GEANT 3.14, CERN Program Library Long Writeup W5013.

[68] A. Gheata et al., The Virtual MonteCarlo: status and applications, Contribution to Computing in High Energy Physics (CHEP2004), September 2004, Interlaken, Switzerland.

[69] S. Aoki et al., The fully automated emulsion analysis system, Nucl. Instrum. Meth. B 51 (1990) 466.

[70] T. Nakano, Automatic analysis of nuclear emulsion, Ph.D. Thesis, Nagoya University, Japan (1997).

[71] G. Rosa, A. Di Bartolomeo, G. Grella and G. Romano, Automatic analysis of digitized TV-images by a computer-driven optical microscope, Nucl. Instrum. Meth. A 394 (1997) 357.

[72] N. Armenise et al., High-speed particle tracking in nuclear emulsion by last- generation automatic microscopes, Nucl. Instrum. Meth. A 551 (2005) 261. 
[73] L. Arrabito et al., Hardware performance of a scanning system for high speed analysis of nuclear emulsions, Nucl. Instrum. Meth. A 568 (2006) 578.

[74] L. Arrabito et al., Track reconstruction in the emulsion-lead target of the OPERA experiment using the ESS microscope, 2007 JINST 2 P05004.

[75] I. Kreslo et al., High-speed analysis of nuclear emulsion films with the use of dry objective lenses, 2008 JINST 3 P04006. 